\title{
Rhodium(III)-Catalyzed Direct C-H Arylation of various Acyclic Enamides with Arylsilanes
}

Xiaolan Li, ${ }^{\dagger}$ Kai Sun, ${ }^{\dagger}$ Wenjuan Shen,${ }^{\dagger}$ Yong Zhang, ${ }^{\dagger}$ Mingzhu Lu, ${ }^{\ddagger}$ Xuzhong Luo, ${ }^{\dagger}$ Haiqing Luo* ${ }^{\dagger}$

†Department of Chemistry \& Chemical Engineering, Gannan Normal University, Ganzhou 341000, China

$\$$ Jiangsu Key Laboratory for Chemistry of Low-Dimensional Materials, School of Chemistry and Chemical Engineering, Huaiyin Normal University, Huaian 223300, China

*E-mail: luohaiq@sina.com

\begin{tabular}{|c|l|}
\hline Table of Contents & Page No. \\
\hline 1. General Information & $\mathrm{S}-2$ \\
\hline 2. Experimental section & $\mathrm{S}-5$ \\
\hline 3. Preliminary mechanistic studies & $\mathrm{S}-7$ \\
\hline 4. $\quad$ Experimental data & $\mathrm{S}-10$ \\
\hline 5. ${ }^{1} \mathrm{H}$ NMR,${ }^{13} \mathrm{C}$ NMR Spectra & $\mathrm{S}-33$ \\
\hline
\end{tabular}




\section{General Information}

\section{Analytical methods}

Proton nuclear magnetic resonance $\left({ }^{1} \mathrm{H}\right.$ NMR $)$ and carbon nuclear magnetic resonance $\left({ }^{13} \mathrm{C}\right.$ NMR) spectroscopy were performed on a Bruker Advance 300, 400 and 500 NMR spectrometers. Chemical shifts ${ }^{1} \mathrm{H}$ NMR spectra are reported as in units of parts per million (ppm) downfield from $\mathrm{SiMe}_{4}(0.0)$ and relative to the signal of chloroform- $d(J=7.264$, singlet $)$. Multiplicities were given as: s (singlet); d (doublet); t (triplet); q (quartet); dd (doublet of doublets); ddd (doublet of doublets of doublets); dddd (doublet of doublets of doublets of doublets); dt (doublet of triplets); $\mathrm{m}$ (multiplets) and etc. The number of protons (n) for a given resonance is indicated by $\mathrm{nH}$. Coupling constants are reported as a $J$ value in $\mathrm{Hz}$. Carbon nuclear magnetic resonance spectra $\left({ }^{13} \mathrm{C}\right.$ NMR) are reported as $\mathrm{d}$ in units of parts per million (ppm) downfield from $\mathrm{SiMe}_{4}(0.0)$ and relative to the signal of chloroform- $d(J=77.03$, triplet). High resolution mass spectral analysis (HRMS) was performed on LCMS Q-TOF (SHIMADZU Corporation) ESI spectrometer. Infrared spectra were recorded on a Nicolet IS50 Fourier transform spectrometer (FT-IR) and are reported in wave numbers $\left(\mathrm{cm}^{-1}\right)$.

\section{Materials}

Unless otherwise noted, all reagents were purchased energy chemistry, Ouhe and Tansoole, and used without further purification. DMF, DMSO, 1, 4-Dioxane and DCE were extra dry bought from energy chemistry.

\section{(1) Synthesis of enamide substrates ${ }^{[1]}$}

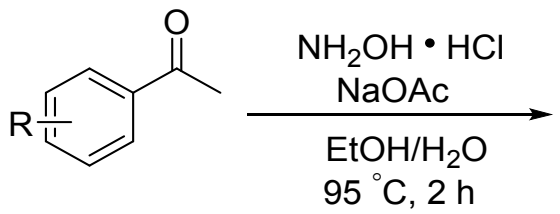<smiles>C=C(NC(=O)O[Na])c1cc[R](C)cc1</smiles>

a) A mixture of ketone (10 mmol, 1.0 equiv), $\mathrm{NaOAc}$ (1.2 equiv), hydroxylamine hydrochloride (1.2 equiv), EtOH $(7.5 \mathrm{~mL})$ and $\mathrm{H}_{2} \mathrm{O}(22.5 \mathrm{~mL})$ was stirred for $2 \mathrm{~h}$ at $95{ }^{\circ} \mathrm{C}$. Then the mixture was evaporated under vacuum and extracted with ethyl acetate. The organic layer was collected, dried over $\mathrm{Na}_{2} \mathrm{SO}_{4}$ and vacuo to afford the ketoxime which was used without further purification for the next step. 
b) To an oven-dried $250 \mathrm{~mL}$ round-bottom flask assembled with condenser was added the above ketoxime, then acetic anhydride ( 2 equiv), sodium bisulfite (3.0 equiv), cuprous iodide ( 0.1 equiv) and DCE $(100 \mathrm{~mL})$ were added. The flask was vacuumed and back filled with $\mathrm{N}_{2}$ for three times. The reaction flask was put into a $120^{\circ} \mathrm{C}$ oil bath for $12 \mathrm{~h}$. After the reaction completed and cooled to room temperature, the organic layer was extracted with ethyl acetate, thus was evaporated to get the crude enamide, which was directly purified by column chromatography.

\section{(2) Synthesis of benzylated enamides ${ }^{[2]}$}

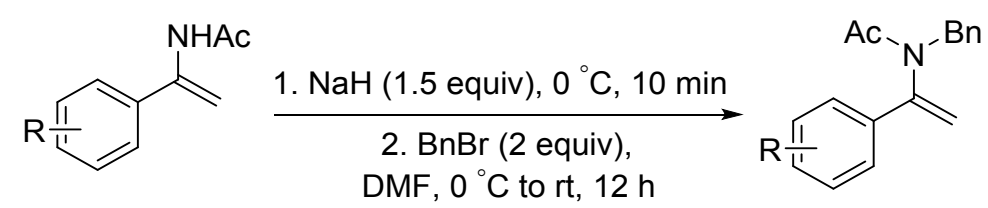

$1.0 \mathrm{mmol}$ (1.0 equiv) of the $N$-acyl enamide was dissolved in $3.0 \mathrm{~mL}$ of dry DMF taken in a dry two-necked flask under nitrogen. The solution was cooled to $0{ }^{\circ} \mathrm{C}$ and $1.5 \mathrm{mmol}$ (1.5 equiv) of sodium hydride was added in portions. The resulting suspension was stirred at the same temperature for $10 \mathrm{~min} .2 .0 \mathrm{mmol}$ (2.0 equiv) of $\mathrm{BnBr}$ was then added dropwise and the mixture was warmed to room temperature overnight. The completion of the reaction was confirmed by checking TLC and the excess of sodium hydride was quenched by adding $1 \mathrm{~mL}$ of water at $0{ }^{\circ} \mathrm{C}$. The organic layer was extracted with ethyl acetate through stages of extraction with water. The combined organic layer was concentrated under reduced pressure and the pure product was isolated by flash column chromatography (Petroleum ether/ethyl acetate $=5 / 1$ ). Enamides including $\mathbf{1 a}, \mathbf{1 b}, \mathbf{1 c}, \mathbf{1 d}, \mathbf{1 e}, \mathbf{1 g}, \mathbf{1 i}, \mathbf{1 j}, \mathbf{1 0}, \mathbf{1 q}, \mathbf{1 r}, \mathbf{1 s}, \mathbf{1 t}, \mathbf{1 u}, \mathbf{1 v}$ are known compounds ${ }^{[3,4]}$, and $\mathbf{1 f}$, $1 \mathrm{~h}, 1 \mathrm{k}, 1 \mathrm{l}, 1 \mathrm{~m}, 1 \mathrm{n}, 1 \mathrm{p}$ are unknown compounds.

\section{(3) Synthesis of various arylsilanes ${ }^{[5]}$}

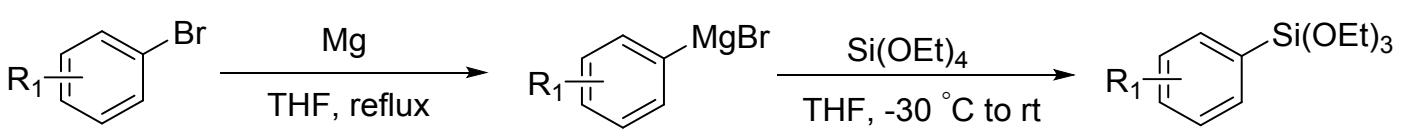

a) Unless otherwise indicated, all reactions were performed on a $30 \mathrm{mmol}$ scale. A $250 \mathrm{~mL}$ three-neck, pear-shaped flask was fitted with an addition funnel, a reflux condenser, a rubber septum, and a stir bar. The flask was then charged with freshly washed magnesium turnings $(0.792$ $\mathrm{g}, 33.0 \mathrm{mmol}$ ), iodine pellets (1 pellet about $10 \mathrm{mg}$ ), and back-filled with nitrogen. Then THF (30 $\mathrm{mL}$ ) was added via syringe. The addition funnel was charged with the aryl halide (30 mmol) in 10 
$\mathrm{mL}$ THF. The reaction was initiated by addition of 5-10 drops of the aryl halide solution to the flask with stirring, followed by gentle heating. The rest of the aryl halide solution was then added and the THF maintained a moderate reflux.

b) A round-bottom flask, which was fitted tetraethyl orthosilicate $(90 \mathrm{mmol})$ in $30 \mathrm{~mL}$ of THF. The silane solution was cooled to $-30{ }^{\circ} \mathrm{C}$, and then the arylmagnesiumhalide solution was added dropwise (one drop per second). The solution was allowed to stir at $-30{ }^{\circ} \mathrm{C}$ for $1 \mathrm{~h}$ and then at room temperature for $12 \mathrm{~h}$. After the reaction finished, the mixture was then poured into $50 \mathrm{~mL}$ of hexane, and stirred for some time. Then the solution was washed with $3 \times 25 \mathrm{~mL}$ of water, dried over $\mathrm{Na}_{2} \mathrm{SO}_{4}$, and concentrated in vacuo. Purification of the residue by micro-pressure distillation could obtain pure aryltriethoxysilane. All the silicon reagent are known compounds. ${ }^{[5,6]}$

\section{(4) Synthesis of deuterated enamide ${ }^{[7]}$}<smiles>C=C(NC(=O)c1ccccc1)c1ccccc1</smiles>

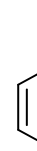<smiles>[2H]C([2H])=C(c1ccccc1)N(Cc1ccccc1)C1CCCCC1</smiles>

$77 \%$ deuterated

a) Sodium hydride (240 mg, $6 \mathrm{mmol}$ ) was added to the solution of enamide (644 mg, $4 \mathrm{mmol}$ ) in dry DMF $(4.0 \mathrm{~mL})$ at $0{ }^{\circ} \mathrm{C}$. The reaction mixture was warmed to $\mathrm{rt}$ and stirred for 8 hours. Then deuterated methanol $(1.0 \mathrm{~mL})$ was added dropwise and the mixture was stirred for 2 hours at $\mathrm{rt}$. The reaction mixture was quenched by addition of water $(10 \mathrm{~mL})$ and extracted with EtOAc for three times, and the resulting organic layers were combined, dried over anhydrous $\mathrm{Na}_{2} \mathrm{SO}_{4}$ and concentrated in reduced pressure. The crude residue was then purified by flash column chromatography on silica gel using petroleum ether/ethyl acetate $(1 / 1)$ to provide the deuterated enamide. Repeat the above experimental procedure for three times to afford the deuterated enamide.

b) $1.0 \mathrm{mmol}$ (1.0 equiv) of the deuterated enamide was dissolved in $3.0 \mathrm{~mL}$ of dry DMF taken in a dry two-necked flask under nitrogen. The solution was cooled to $0{ }^{\circ} \mathrm{C}$ and $1.5 \mathrm{mmol}$ (1.5 equiv) of sodium hydride was added in portions. The resulting suspension was stirred at the same temperature for $10 \mathrm{~min} .2 .0 \mathrm{mmol}$ (2.0 equiv) of $\mathrm{BnBr}$ was then added dropwise and the mixture was warmed to room temperature overnight. The completion of the reaction was confirmed by 
checking TLC and the excess of sodium hydride was quenched by adding $1 \mathrm{~mL}$ of water at $0{ }^{\circ} \mathrm{C}$. The organic layer was extracted with ethyl acetate through stages of extraction with water. The combined organic layer was concentrated under reduced pressure and the $77 \%$ deuterated 1a was isolated by flash column chromatography (Petroleum ether/ethyl acetate $=5 / 1)$.

\section{2、 Experimental section}

\subsection{General procedure for synthesis of arylated enamides}

To a $15 \mathrm{~mL}$ of seal tube equipped with a magnetic bar were added enamide $(0.27 \mathrm{mmol}, 1$ equiv), $\left[\mathrm{Cp}^{*} \mathrm{RhCl}_{2}\right]_{2}(0.0054 \mathrm{mmol}, 2 \mathrm{~mol} \%)$ and $\mathrm{CuF}_{2}(0.81 \mathrm{mmol}, 3.0$ equiv). The DMF $(2.0$ $\mathrm{mL})$ and arylsilane $(0.81 \mathrm{mmol}, 3.0$ equiv) were added subsequently. The reaction mixture was allowed to stir at $100{ }^{\circ} \mathrm{C}$ (oil bath) for $24 \mathrm{~h}$. After the reaction finished, the mixture was cooled to room temperature, then diluted with $10 \mathrm{~mL}$ water and extracted with EtOAc $(3 * 10 \mathrm{~mL})$. The combined organic layers were washed with brine, dried over $\mathrm{Na}_{2} \mathrm{SO}_{4}$, evaporated under vacuum. The residue was purified with silica gel chromatography (petroleum ether/ethyl acetate) to provide pure product.

\subsection{Gram-scale synthesis of arylated enamides}

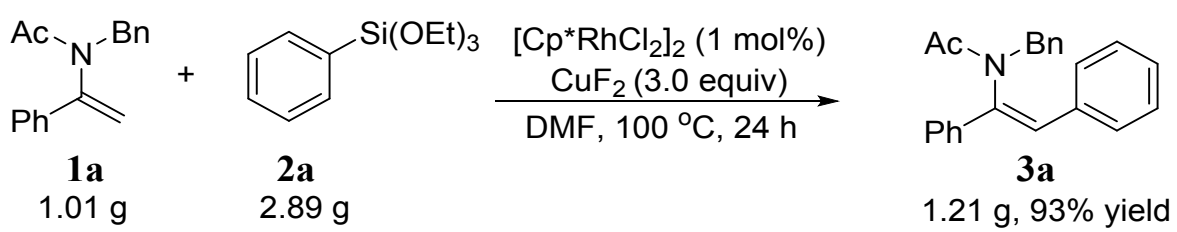

To a $25 \mathrm{~mL}$ of flask equipped with a magnetic bar were added enamide 1a $(4.0 \mathrm{mmol}, 1.0$ equiv), $\left[\mathrm{Cp}^{*} \mathrm{RhCl}_{2}\right]_{2}(0.04 \mathrm{mmol}, 1 \mathrm{~mol} \%)$ and $\mathrm{CuF}_{2}(12 \mathrm{mmol}, 3.0$ equiv). The DMF (20 mL) and arylsilane $\mathbf{2 a}$ (12.0 mmol, 3.0 equiv) were added subsequently. The reaction mixture was allowed to stir at $100{ }^{\circ} \mathrm{C}$ (oil bath) for $24 \mathrm{~h}$. After the reaction finished, the mixture was cooled to room temperature, then diluted with water and extracted with EtOAc. The combined organic layers were washed with brine, dried over $\mathrm{Na}_{2} \mathrm{SO}_{4}$, evaporated under vacuum. The residue was purified with silica gel chromatography (petroleum ether/ethyl acetate) to provide pure product. 


\subsection{Transformations of 3a}

a) Hydrogenation of 3a<smiles>O=C(c1ccccc1)N(Cc1ccccc1)c1ccccc1</smiles>

3a

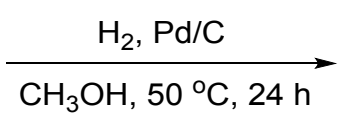

$\mathrm{CH}_{3} \mathrm{OH}, 50^{\circ} \mathrm{C}, 24 \mathrm{~h}$<smiles>CN(Cc1ccccc1)C(Cc1ccccc1C(C)(C)C)c1ccccc1</smiles>

$5,88 \%$ yield

(Z)- $N$-benzyl- $N$-(1,2-diphenylvinyl)acetamide 3a $(0.2 \mathrm{mmol}, 65.49 \mathrm{mg})$ in methanol (3.0 $\mathrm{ml})$ was hydrogenated at $50^{\circ} \mathrm{C}$ under room pressure in the presence of $\mathrm{Pd} / \mathrm{C}(6.5 \mathrm{mg})$ for $24 \mathrm{~h}$. Upon completion, the solvent was then removed under vacuum. The residue was purified directly by silica gel chromatography, eluting with ethyl acetate/petroleum ether (1:10 v/v). $N$-benzyl- $N$-(1,2-diphenylethyl)acetamide 5 was obtained in $88 \%$ yield $(58.0 \mathrm{mg})$ as a colorless oil.

\section{b) $\mathrm{C}-\mathrm{H}$ arylation of $3 \mathrm{a}$}<smiles>O=C(Cl)N(Cc1ccccc1)/C(=C/c1ccccc1)c1ccccc1</smiles>

3a

$$
\begin{gathered}
\mathrm{Pd}(\mathrm{OAc})_{2}(10 \text { mol\% }) \\
\mathrm{Cu}(\mathrm{OTf})_{2}(2.0 \text { equiv }) \\
\mathrm{PhB}(\mathrm{OH})_{2}(2.0 \text { equiv })
\end{gathered}
$$<smiles></smiles>

6, $61 \%$ yield

To a $15 \mathrm{~mL}$ of seal tube equipped with a magnetic bar were added $\mathbf{3 a}(0.2 \mathrm{mmol}, 1.0$ equiv), $\mathrm{Pd}(\mathrm{OAc})_{2}(10 \mathrm{~mol} \%), \mathrm{Cu}(\mathrm{OTf})_{2}\left(2.0\right.$ equiv), $\mathrm{PhB}(\mathrm{OH})_{2}$ (2.0 equiv) and $\mathrm{K}_{3} \mathrm{PO}_{4}$ (2.0 equiv). The dioxane $(2.0 \mathrm{~mL})$ were added subsequently. The reaction mixture was allowed to stir at $110^{\circ} \mathrm{C}$ (oil bath) for $30 \mathrm{~h}$. After the reaction finished, the mixture was cooled to room temperature, then diluted with $10 \mathrm{~mL}$ water and extracted with EtOAc $(3 * 10 \mathrm{~mL})$. The combined organic layers were washed with brine, dried over $\mathrm{Na}_{2} \mathrm{SO}_{4}$, evaporated under vacuum. The residue was purified with silica gel chromatography to provide the tetrasubstituted enamide product 6 in $61 \%$ yield.

\subsection{Reaction between the enamide and phenyl boronic acid under identical conditions}




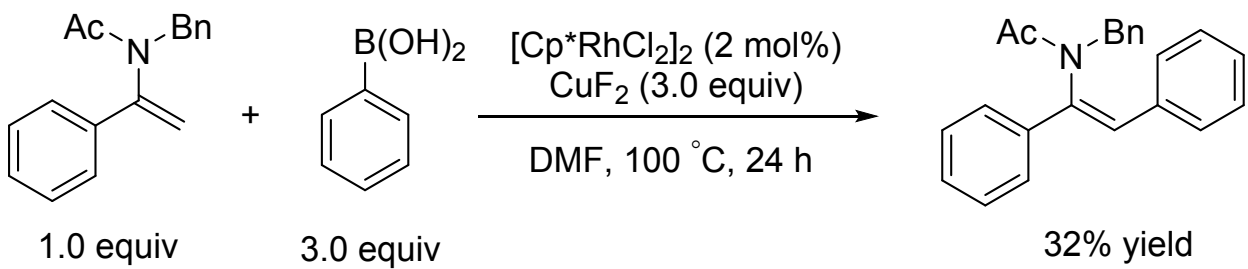

The reaction between the enamide and phenyl boronic acid under almost identical conditions to the direct arylation reaction has led to the formation of the $Z$-arylated product with yield of $32 \%$. The reaction conditions were not optimized.

\subsection{The method of Crystal Synthesis}

Synthesis was carried out by reaction of $\mathbf{1 a}(0.27 \mathrm{mmol})$ and $\mathbf{2 a}(0.81 \mathrm{mmol})$ in DMF at 100 ${ }^{\circ} \mathrm{C}$ for $24 \mathrm{~h}$ in a reaction tube. Single crystals suitable for X-ray analysis were grown from ethyl acetate solution of $\mathbf{3 a}$ by slow vapor diffusion of petroleum ether.

\section{3、Preliminary mechanistic studies}

\subsection{Mechanism studies}

\section{a) Competitive reaction}

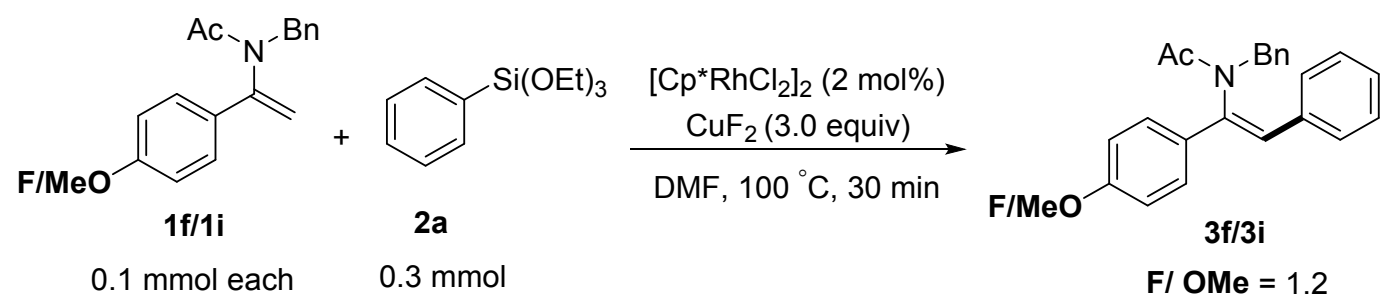

$0.1 \mathrm{mmol}$ (28.2 $\mathrm{mg}, 1$ equiv) of the compound $\mathbf{1 f}$ and $0.1 \mathrm{mmol}(26.9 \mathrm{mg}, 1.0$ equiv) of the compound $\mathbf{1} \mathbf{i}$ were dissolved in $2.0 \mathrm{~mL}$ of DMF in a seal tube equipped with a magnetic bar. 0.002 mmol (1.4 mgl, $2 \mathrm{~mol} \%)$ of $\left[\mathrm{Cp}^{*} \mathrm{RhCl}_{2}\right]_{2}, 0.3 \mathrm{mmol}(31.1 \mathrm{mg}, 3.0$ equiv) of the copper fluoride and $0.3 \mathrm{mmol}$ phenyltriethoxysilane were added. The solution was heated at $100{ }^{\circ} \mathrm{C}$ for 30 minutes. After the reaction finished, the mixture was removed in vacuum and the product was pale yellow oil. The $\mathbf{F} / \mathbf{O M e}=\mathbf{1 . 2}$ was determined from the ${ }^{1} \mathrm{H}-\mathrm{NMR}$.

\section{b) Intermolecular kinetic isotopic effect (KIE) study}


$18.0 \mathrm{mg}(0.0716 \mathrm{mmol})$ of the protonated enamide and $33.3 \mathrm{mg}(0.1315 \mathrm{mmol})$ of the deuterated analogue were dissolved in $2.0 \mathrm{~mL}$ of DMF in a seal tube equipped with a magnetic bar. $2.5 \mathrm{mg}(0.004 \mathrm{mmol}, 2 \mathrm{~mol} \%)$ of $\left[\mathrm{Cp}^{*} \mathrm{RhCl}_{2}\right]_{2}, 62.2 \mathrm{mg}(0.6 \mathrm{mmol})$ of the copper fluoride and $148 \mu \mathrm{L}$ phenyltriethoxysilane were added. The solution was heated at $100{ }^{\circ} \mathrm{C}$ for 5 minutes. After the reaction finished, the mixture was removed in vacuum and the product was isolated through flash column chromatography (petroleum ether/ethyl acetate)) to furnish $9.0 \mathrm{mg}(14 \%)$ of the product mixture as pale yellow oil. In order to check the KIE value, the same reaction was carried out for 10 minutes, and $15.8 \mathrm{mg}(18 \%)$ of the product was obtained. The $K I E$ value $\left[k_{\mathrm{H}} / k_{\mathrm{D}}\right.$ $($ average $)=1.25]$ was determined from the ${ }^{1} \mathrm{H}-\mathrm{NMR}$.<smiles>C1=CCCCC1</smiles><smiles>[2H]C([2H])=C(c1ccccc1)N(Cc1ccccc1)C(=O)O</smiles>

$0.132 \mathrm{mmol}$ $77 \%$ deuterium

(i) Reaction time $=5 \mathrm{~min}$

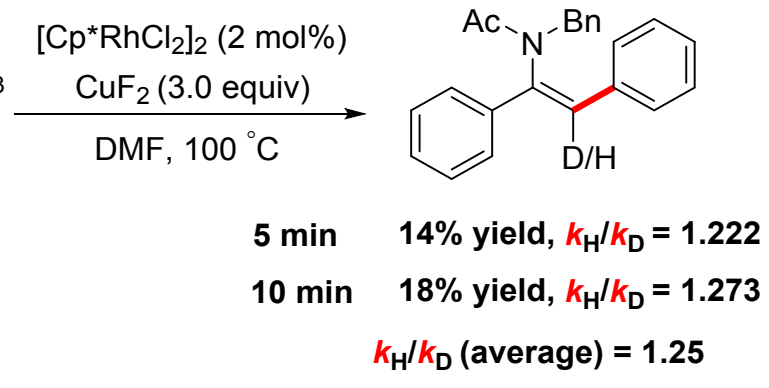

$0.6 \mathrm{mmol}$

$5 \mathrm{~min} \quad 14 \%$ yield, $k_{\mathrm{H}} / k_{\mathrm{D}}=1.222$

$k_{H} / k_{D}($ average $)=1.25$ 


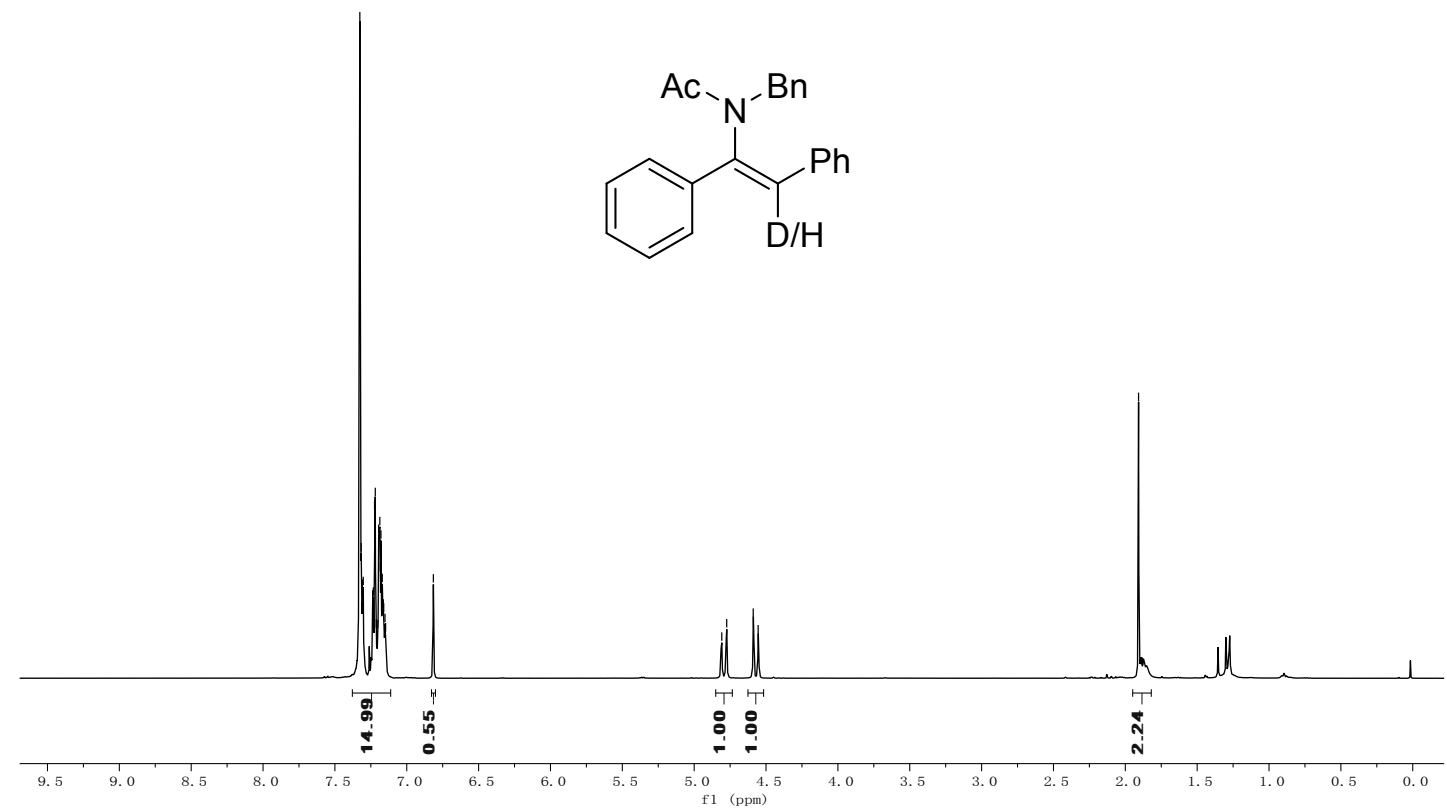

(ii) Reaction time $=10 \mathrm{~min}$

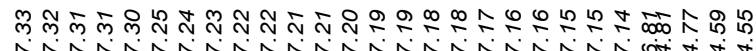

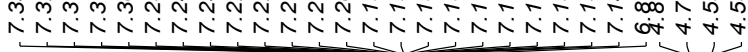

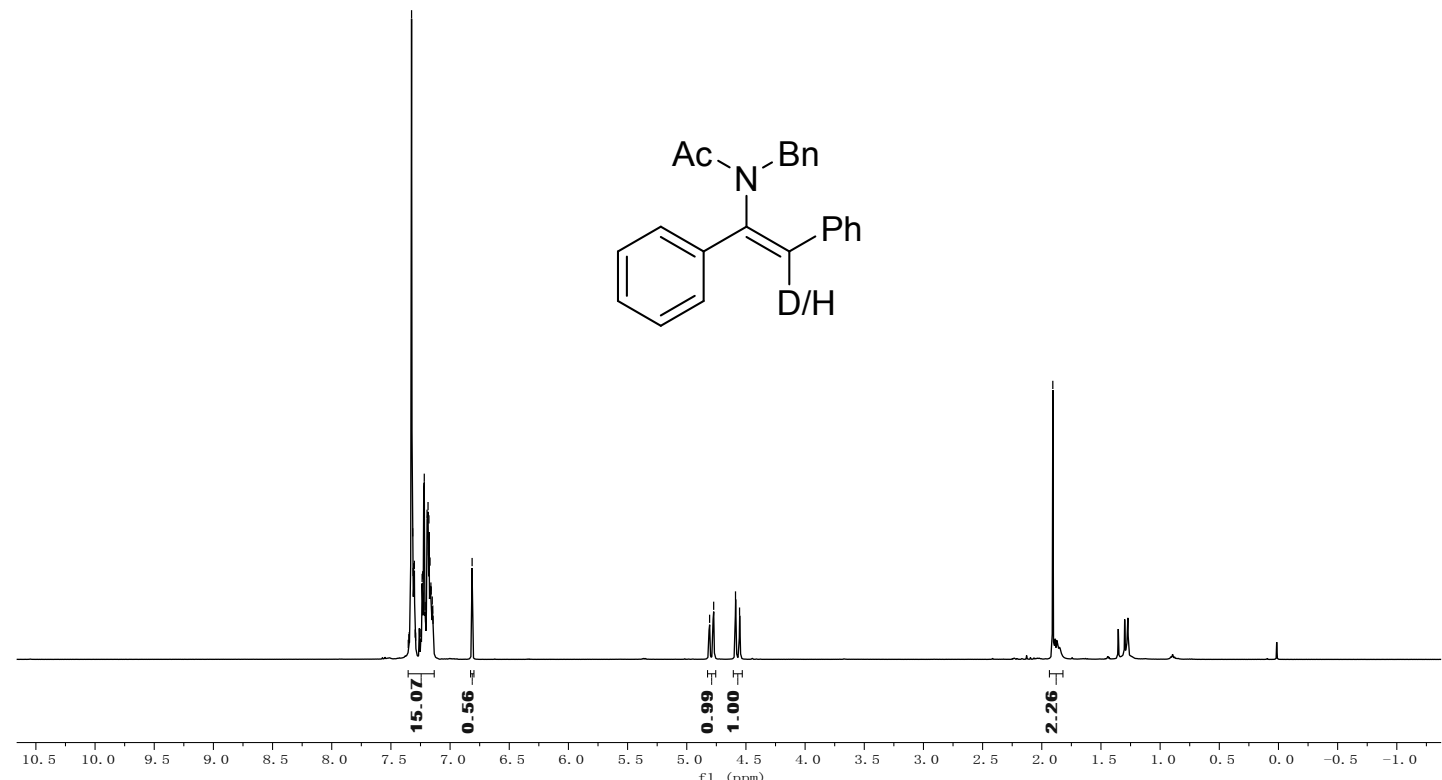

Spectra of the product mixture for the determination of KIE 


\section{4、 Experimental data}<smiles>C=C(c1ccc(OC)cc1OC)N(Cc1ccccc1)C(C)=O</smiles>

$N$-benzyl- $N$-(1-(2,4-dimethoxyphenyl)vinyl)acetamide (1f)

Following the general procedure (eluent: petroleum ether/ethyl acetate $=5 / 1$ ), the desired product 1f was obtained as a pale yellow oil; ${ }^{1} \mathbf{H}$ NMR $\left(400 \mathrm{MHz}, \mathrm{CDCl}_{3}\right) \delta[\mathrm{ppm}]=7.28-7.17(\mathrm{~m}, 5 \mathrm{H})$, $7.06(\mathrm{~d}, J=9.1 \mathrm{~Hz}, 1 \mathrm{H}), 6.54-6.38(\mathrm{~m}, 2 \mathrm{H}), 5.46(\mathrm{~s}, 1 \mathrm{H}), 4.92(\mathrm{~s}, 1 \mathrm{H}), 4.54(\mathrm{~s}, 2 \mathrm{H}), 3.81(\mathrm{~s}, 3 \mathrm{H})$, 3.77 (s, 3H), 2.19 (s, 3H). ${ }^{13} \mathbf{C}$ NMR (101 MHz, $\left.\mathrm{CDCl}_{3}\right) \delta[\mathrm{ppm}]=171.0,161.3,158.7,144.3$, $137.8,130.8,128.6,127.9,126.8,117.4,116.2,104.3,98.7,55.3,55.3,49.0,22.2$.

IR (KBr) v (cm (c) $^{-1}$ 3062, 3029, 2937, 2838, 1606, 1456, 1391, 1295, 1031, 836, 700.

HRMS (ESI) m/z: $[\mathrm{M}+\mathrm{H}]^{+}$Calcd for $\mathrm{C}_{19} \mathrm{H}_{22} \mathrm{NO}_{3} 312.1594$; found 312.1600 .<smiles>C=C(c1ccc2c(c1)OCO2)N(Cc1ccccc1)C(C)=O</smiles>

$1 \mathrm{~h}$

$N$-(1-(benzo[d][1,3]dioxol-5-yl)vinyl)-N-benzylacetamide (1h)

Following the general procedure (eluent: petroleum ether/ethyl acetate $=5 / 1$ ), the desired product 1h was obtained as a pale yellow oil; ${ }^{\mathbf{1}} \mathbf{H} \mathbf{N M R}\left(400 \mathrm{MHz}, \mathrm{CDCl}_{3}\right) \delta[\mathrm{ppm}]=7.25$ (hept, $J=2.4$ Hz, 5H), $6.87-6.82(\mathrm{~m}, 2 \mathrm{H}), 6.78(\mathrm{~d}, J=8.5 \mathrm{~Hz}, 1 \mathrm{H}), 5.97(\mathrm{~s}, 2 \mathrm{H}), 5.45(\mathrm{~s}, 1 \mathrm{H}), 4.76(\mathrm{~s}, 1 \mathrm{H})$, $4.63(\mathrm{~s}, 2 \mathrm{H}), 2.08(\mathrm{~s}, 3 \mathrm{H}) .{ }^{13} \mathrm{C}$ NMR $\left(101 \mathrm{MHz}, \mathrm{CDCl}_{3}\right) \delta[\mathrm{ppm}]=170.6,148.4,148.3,145.9$, 137.4, 129.6, 128.9, 128.1, 127.2, 119.8, 113.1, 108.4, 106.0, 101.4, 49.7, 21.8.

IR (KBr) v (cm-1) 3063, 3030, 2900, 1657, 1489, 1390, 1245, 1036, 979, 700.

HRMS (ESI) m/z: [M+H] $]^{+}$Calcd for $\mathrm{C}_{18} \mathrm{H}_{18} \mathrm{NO}_{3}$ 296.1281; found 296.1285. 
<smiles>C=C(c1ccc(Br)cc1)N(Cc1ccccc1)C(C)=O</smiles>

$N$-benzyl- $N$-(1-(4-bromophenyl)vinyl)acetamide (1k)

Following the general procedure (eluent: petroleum ether/ethyl acetate $=5 / 1$ ), the desired product 1k was obtained as a pale yellow oil; ${ }^{1} \mathbf{H}$ NMR $\left(400 \mathrm{MHz}, \mathrm{CDCl}_{3}\right) \delta[\mathrm{ppm}]=7.50-7.42(\mathrm{~m}, 2 \mathrm{H})$, $7.27-7.15(\mathrm{~m}, 7 \mathrm{H}), 5.58(\mathrm{~s}, 1 \mathrm{H}), 4.89(\mathrm{~s}, 1 \mathrm{H}), 4.61(\mathrm{~s}, 2 \mathrm{H}), 2.04(\mathrm{~s}, 3 \mathrm{H}) .{ }^{13} \mathbf{C}$ NMR $(101 \mathrm{MHz}$ $\left.\mathrm{CDCl}_{3}\right) \delta[\mathrm{ppm}]=170.3,145.3,137.0,134.2,131.8,128.7,128.1,127.2,127.1,123.0,114.9$, 49.6, 21.7 .

IR (KBr) v (cm-1) 3062, 3030, 2929, 1659, 1486, 1390, 1235, 1030, 979, 768, 699.

HRMS (ESI) m/z: $[\mathrm{M}+\mathrm{H}]^{+}$Calcd for $\mathrm{C}_{17} \mathrm{H}_{17} \mathrm{BrNO} 330.0488$; found 330.0493 .<smiles>C=C(c1cccc(Br)c1)N(Cc1ccccc1)C(C)=O</smiles>

1 I

$N$-benzyl- $N$-(1-(3-bromophenyl)vinyl)acetamide (11)

Following the general procedure (eluent: petroleum ether/ethyl acetate $=5 / 1$ ), the desired product 11 was obtained as a pale yellow oil; ${ }^{1} \mathbf{H}$ NMR $\left(400 \mathrm{MHz}, \mathrm{CDCl}_{3}\right) \delta[\mathrm{ppm}]=7.55-7.46(\mathrm{~m}, 2 \mathrm{H})$, $7.33-7.20(\mathrm{~m}, 7 \mathrm{H}), 5.62(\mathrm{~s}, 1 \mathrm{H}), 4.93(\mathrm{~s}, 1 \mathrm{H}), 4.64(\mathrm{~s}, 2 \mathrm{H}), 2.08(\mathrm{~s}, 3 \mathrm{H}) .{ }^{13} \mathrm{C}$ NMR $(101 \mathrm{MHz}$ $\left.\mathrm{CDCl}_{3}\right) \delta[\mathrm{ppm}]=170.5,145.2,137.6,137.2,132.0,130.4,128.9,128.7,128.3,127.4,124.3$, $123.1,115.8,49.8,2$.

IR (KBr) v (cm (c) $^{-1}$ 3062, 3030, 2929, 1661, 1494, 1387, 1234, 1070, 978, 792, 698.

HRMS (ESI) m/z: $[\mathrm{M}+\mathrm{H}]^{+}$Calcd for $\mathrm{C}_{17} \mathrm{H}_{17} \mathrm{BrNO} 330.0488$; found 330.0493 .<smiles>C=C(c1ccccc1Br)N(Cc1ccccc1)C(C)=O</smiles> 
$N$-benzyl- $N$-(1-(2-bromophenyl)vinyl)acetamide (1 m)

Following the general procedure (eluent: petroleum ether/ethyl acetate $=5 / 1$ ), the desired product 1m was obtained as a pale yellow oil; ${ }^{\mathbf{1}} \mathbf{H} \mathbf{N M R}\left(400 \mathrm{MHz}, \mathrm{CDCl}_{3}\right) \delta[\mathrm{ppm}]=7.61(\mathrm{~d}, J=7.8 \mathrm{~Hz}$, 1H), $7.33-7.13(\mathrm{~m}, 7 \mathrm{H}), 5.36(\mathrm{~s}, 1 \mathrm{H}), 5.17(\mathrm{~s}, 1 \mathrm{H}), 4.63(\mathrm{~d}, J=58.4 \mathrm{~Hz}, 2 \mathrm{H}), 2.32(\mathrm{~s}, 3 \mathrm{H}) .{ }^{13} \mathrm{C}$ $\operatorname{NMR}\left(101 \mathrm{MHz}, \mathrm{CDCl}_{3}\right) \delta[\mathrm{ppm}]=171.0,145.9,137.3,136.6,133.8,131.5,130.1,128.3,128.1$, $127.5,127.0,121.6,117.0,49.0,22.8$.

IR (KBr) v (cm (c) $^{-1}$ 3062, 3029, 2932, 1658, 1494, 1384, 1234, 1025, 982, 766, 698.

HRMS (ESI) m/z: $[\mathrm{M}+\mathrm{H}]^{+}$Calcd for $\mathrm{C}_{17} \mathrm{H}_{17} \mathrm{BrNO} 330.0488$; found 330.0493 .<smiles>C=C(c1ccc(C(F)(F)F)cc1)N(Cc1ccccc1)C(C)=O</smiles>

N-benzyl-N-(1-(4-(trifluoromethyl)phenyl)vinyl)acetamide (1n)

Following the general procedure (eluent: petroleum ether/ethyl acetate $=5 / 1$ ), the desired product 1n was obtained as a pale yellow oil; ${ }^{1} \mathbf{H}$ NMR $\left(400 \mathrm{MHz}, \mathrm{CDCl}_{3}\right) \delta[\mathrm{ppm}]=7.62(\mathrm{~d}, J=8.3 \mathrm{~Hz}$, 2H), $7.48(\mathrm{~d}, J=8.2 \mathrm{~Hz}, 2 \mathrm{H}), 7.31-7.19(\mathrm{~m}, 5 \mathrm{H}), 5.72(\mathrm{~s}, 1 \mathrm{H}), 5.02(\mathrm{~s}, 1 \mathrm{H}), 4.65(\mathrm{~s}, 2 \mathrm{H}), 2.07$ (s, 3H). ${ }^{13} \mathbf{C ~ N M R ~}\left(101 \mathrm{MHz}, \mathrm{CDCl}_{3}\right) \delta[\mathrm{ppm}]=170.5,145.3,138.9,137.0,130.8\left(\mathrm{~d},{ }^{2} J_{C-F}=32.9\right.$ $\mathrm{Hz}), 128.8,128.2,127.4,126.0,125.8\left(\mathrm{q},{ }^{3} J_{C-F}=3.8 \mathrm{~Hz}\right), 123.7\left(\mathrm{~d},{ }^{1} J_{C-F}=272.2 \mathrm{~Hz}\right), 116.6,49.8$, 21.8 .

IR (KBr) v (cm') 3064, 3032, 2932, 1667, 1494, 1390, 1235, 1030, 979, 750, 699.

HRMS (ESI) m/z: $[\mathrm{M}+\mathrm{H}]^{+}$Calcd for $\mathrm{C}_{18} \mathrm{H}_{17} \mathrm{~F}_{3} \mathrm{NO} 320.1257$; found 320.1263 .<smiles>C=C(c1ccc([N+](=O)[O-])cc1)N(Cc1ccccc1)C(C)=O</smiles>

$N$-benzyl- $N$-(1-(4-nitrophenyl)vinyl)acetamide (1p)

Following the general procedure (eluent: petroleum ether/ethyl acetate $=5 / 1$ ), the desired product 
1p was obtained as a pale yellow oil; ${ }^{1} \mathbf{H}$ NMR $\left(400 \mathrm{MHz}, \mathrm{CDCl}_{3}\right) \delta[\mathrm{ppm}]=8.19(\mathrm{~d}, J=8.7 \mathrm{~Hz}$, 2H), $7.66-7.37(\mathrm{~m}, 2 \mathrm{H}), 7.35-7.08(\mathrm{~m}, 5 \mathrm{H}), 5.81(\mathrm{~s}, 1 \mathrm{H}), 5.13(\mathrm{~s}, 1 \mathrm{H}), 4.66(\mathrm{~s}, 2 \mathrm{H}), 2.06(\mathrm{~s}$, 3H). ${ }^{13} \mathbf{C}$ NMR $\left(101 \mathrm{MHz}, \mathrm{CDCl}_{3}\right) \delta[\mathrm{ppm}]=170.3,147.9,144.8,141.8,136.8,128.9,128.3$, $127.5,126.5,124.1,118.3,50.0,21.9$.

IR (KBr) v (cm $\left.\mathbf{c m}^{-1}\right)$ 3060, 3025, 2849, 1657, 1517, 1392, 1235, 1031, 978, 698.

HRMS (ESI) m/z: $[\mathrm{M}+\mathrm{H}]^{+}$Calcd for $\mathrm{C}_{17} \mathrm{H}_{17} \mathrm{~N}_{2} \mathrm{O}_{3} 297.1234$; found 297.1237 .<smiles>CC(=O)N(Cc1ccccc1)/C(=C\c1ccccc1)c1ccccc1</smiles>

$3 a$

(Z)- $N$-benzyl- $N$-(1,2-diphenylvinyl)acetamide (3a)

Following the general procedure (eluent: petroleum ether/ethyl acetate $=10 / 1$ ), the desired product 3a was obtained as a pale yellow oil $\left(81.0 \mathrm{mg}, 92 \%\right.$ yield); ${ }^{1} \mathbf{H}$ NMR $\left(400 \mathrm{MHz}, \mathrm{CDCl}_{3}\right) \delta[\mathrm{ppm}]$ $=7.35-7.29(\mathrm{~m}, 7 \mathrm{H}), 7.22(\mathrm{p}, J=2.4 \mathrm{~Hz}, 3 \mathrm{H}), 7.21-7.14(\mathrm{~m}, 5 \mathrm{H}), 6.81(\mathrm{~s}, 1 \mathrm{H}), 4.79(\mathrm{~d}, J=$ $13.7 \mathrm{~Hz}, 1 \mathrm{H}), 4.57(\mathrm{~d}, J=13.8 \mathrm{~Hz}, 1 \mathrm{H}), 1.91(\mathrm{~s}, 3 \mathrm{H}) .{ }^{13} \mathbf{C} \mathbf{N M R}\left(101 \mathrm{MHz}, \mathrm{CDCl}_{3}\right) \delta[\mathrm{ppm}]=$ $171.0,139.2,137.9,136.5,134.6,130.3,128.8,128.7,128.6,128.3,128.2,128.2,127.6,126.9$, 126.1, 50.3, 22.0. This compound is known. ${ }^{[3]}$<smiles>CC(=O)N(Cc1ccccc1)/C(=C\c1ccccc1)c1ccc(C)cc1</smiles>

$3 b$

(Z)- $N$-benzyl- $N$-(2-phenyl-1-(p-tolyl)vinyl)acetamide (3b)

Following the general procedure (eluent: petroleum ether/ethyl acetate $=20 / 1$ ), the desired product 3b was obtained as a pale yellow oil $\left(80.0 \mathrm{mg}, 87 \%\right.$ yield); ${ }^{1} \mathbf{H}$ NMR $\left(400 \mathrm{MHz}, \mathrm{CDCl}_{3}\right) \delta[\mathrm{ppm}]$ $=7.38-7.30(\mathrm{~m}, 2 \mathrm{H}), 7.23(\mathrm{~d}, J=1.8 \mathrm{~Hz}, 2 \mathrm{H}), 7.22-7.18(\mathrm{~m}, 6 \mathrm{H}), 7.15(\mathrm{dt}, J=6.5,2.3 \mathrm{~Hz}, 4 \mathrm{H})$, $6.79(\mathrm{~s}, 1 \mathrm{H}), 4.80(\mathrm{~d}, J=13.7 \mathrm{~Hz}, 1 \mathrm{H}), 4.58(\mathrm{~d}, J=13.7 \mathrm{~Hz}, 1 \mathrm{H}), 2.38(\mathrm{~s}, 3 \mathrm{H}), 1.92(\mathrm{~s}, 3 \mathrm{H}) .{ }^{13} \mathrm{C}$ 
NMR $\left(101 \mathrm{MHz}, \mathrm{CDCl}_{3}\right) \delta[\mathrm{ppm}]=171.0,139.2,139.0,136.6,135.0,134.7,130.3,129.4,128.6$, $128.1,128.1,128.1,127.5,126.0,126.0,50.3,22.0,21.1$. This compound is known. ${ }^{[3]}$<smiles>COc1ccc(/C(=C/c2ccccc2)N(Cc2ccccc2)C(C)=O)cc1</smiles>

(Z)-N-benzyl- $N$-(1-(4-methoxyphenyl)-2-phenylvinyl)acetamide (3c)

Following the general procedure (eluent: petroleum ether/ethyl acetate $=20 / 1$ ), the desired product 3c was obtained as a pale yellow oil ( $83.5 \mathrm{mg}, 87 \%$ yield); ${ }^{1} \mathbf{H}$ NMR $\left(400 \mathrm{MHz}, \mathrm{CDCl}_{3}\right) \delta[\mathrm{ppm}]$ $=7.35-7.29(\mathrm{~m}, 2 \mathrm{H}), 7.25-7.13(\mathrm{~m}, 10 \mathrm{H}), 6.85(\mathrm{~d}, J=8.9 \mathrm{~Hz}, 2 \mathrm{H}), 6.71(\mathrm{~s}, 1 \mathrm{H}), 4.72(\mathrm{~d}, J=$ $13.7 \mathrm{~Hz}, 1 \mathrm{H}), 4.64(\mathrm{~d}, J=13.7 \mathrm{~Hz}, 1 \mathrm{H}), 3.81(\mathrm{~s}, 3 \mathrm{H}), 1.91(\mathrm{~s}, 3 \mathrm{H}) .{ }^{13} \mathbf{C} \mathbf{N M R}\left(101 \mathrm{MHz}, \mathrm{CDCl}_{3}\right) \delta$ $[\mathrm{ppm}]=171.0,159.9,138.9,136.6,134.8,130.3,130.3,128.7,128.1,128.0,127.9,127.6,127.4$ $124.9,114.1,55.3,50.3,22.0$. This compound is known. ${ }^{[3]}$<smiles>COc1cccc(/C(=C/c2ccccc2)N(Cc2ccccc2)C(C)=O)c1</smiles>

3d

(Z)-N-benzyl- $N$-(1-(3-methoxyphenyl)-2-phenylvinyl)acetamide (3d)

Following the general procedure (eluent: petroleum ether/ethyl acetate $=15 / 1$ ), the desired product 3d was obtained as a pale yellow oil ( $84.1 \mathrm{mg}, 87 \%$ yield); ${ }^{1} \mathbf{H}$ NMR $\left(400 \mathrm{MHz}, \mathrm{CDCl}_{3}\right) \delta[\mathrm{ppm}]$ $=7.34(\mathrm{~s}, 7 \mathrm{H}), 7.21-7.13(\mathrm{~m}, 4 \mathrm{H}), 6.79(\mathrm{dt}, J=5.6,1.7 \mathrm{~Hz}, 4 \mathrm{H}), 4.79(\mathrm{~d}, J=13.8 \mathrm{~Hz}, 1 \mathrm{H}), 4.57$ $(\mathrm{d}, J=13.7 \mathrm{~Hz}, 1 \mathrm{H}), 3.64(\mathrm{~s}, 3 \mathrm{H}), 1.92(\mathrm{~s}, 3 \mathrm{H}) .{ }^{13} \mathbf{C}$ NMR $(101 \mathrm{MHz}, \mathrm{CDCl} 3) \delta[\mathrm{ppm}]=171.1$, 159.7, 139.4, 137.87, 136.5, 135.9, 130.3, 129.6, 128.7, 128.6, 128.2, 127.6, 126.8, 126.1, 121.1, $114.8,112.5,55.1,50.4,22.0$. This compound is known. ${ }^{[3]}$ 
<smiles>COc1ccccc1/C(=C/c1ccccc1)N(Cc1ccccc1)C(C)=O</smiles>

$3 e$

(Z)- $N$-benzyl- $N$-(1-(2-methoxyphenyl)-2-phenylvinyl)acetamide (3e)

Following the general procedure (eluent: petroleum ether/ethyl acetate $=10 / 1$ ), the desired product 3e was obtained as a pale yellow oil $\left(83.3 \mathrm{mg}, 86 \%\right.$ yield); ${ }^{1} \mathbf{H}$ NMR $\left(400 \mathrm{MHz}, \mathrm{CDCl}_{3}\right) \delta[\mathrm{ppm}]$ $=7.35-7.25(\mathrm{~m}, 4 \mathrm{H}), 7.18(\mathrm{~d}, J=2.5 \mathrm{~Hz}, 6 \mathrm{H}), 7.13-7.06(\mathrm{~m}, 2 \mathrm{H}), 6.98(\mathrm{t}, J=7.4 \mathrm{~Hz}, 1 \mathrm{H}), 6.83$ (d, $J=8.2 \mathrm{~Hz}, 1 \mathrm{H}), 6.60(\mathrm{~s}, 1 \mathrm{H}), 4.90(\mathrm{~d}, J=13.9 \mathrm{~Hz}, 1 \mathrm{H}), 4.36(\mathrm{~d}, J=13.9 \mathrm{~Hz}, 1 \mathrm{H}), 3.72(\mathrm{~s}, 3 \mathrm{H})$, $1.98(\mathrm{~s}, 3 \mathrm{H}) .{ }^{13} \mathrm{C}$ NMR $\left(101 \mathrm{MHz}, \mathrm{CDCl}_{3}\right) \delta[\mathrm{ppm}]=171.1,157.6,137.9,136.9,135.0,130.8$, $130.0,129.8,129.1,128.5,127.8,127.7,127.1,126.6,120.5,110.7,55.0,49.6,21.8$.

This compound is known. ${ }^{[3]}$<smiles>COc1ccc(/C(=C/c2ccccc2)N(Cc2ccccc2)C(C)=O)c(OC)c1</smiles>

$3 f$

(Z)- $N$-benzyl- $N$-(1-(2,4-dimethoxyphenyl)-2-phenylvinyl)acetamide (3f)

Following the general procedure (eluent: petroleum ether/ethyl acetate $=10 / 1$ ), the desired product 3f was obtained as a pale yellow oil $(100.1 \mathrm{mg}, 96 \%$ yield $) ;{ }^{1} \mathbf{H} \mathbf{~ N M R}\left(400 \mathrm{MHz}, \mathrm{CDCl}_{3}\right) \delta[\mathrm{ppm}]$ $=7.32-7.23(\mathrm{~m}, 2 \mathrm{H}), 7.22-7.13(\mathrm{~m}, 7 \mathrm{H}), 7.09-7.01(\mathrm{~m}, 2 \mathrm{H}), 6.56-6.43(\mathrm{~m}, 2 \mathrm{H}), 6.38(\mathrm{~d}, J=$ $2.4 \mathrm{~Hz}, 1 \mathrm{H}), 4.85(\mathrm{~d}, J=13.9 \mathrm{~Hz}, 1 \mathrm{H}), 4.35(\mathrm{~d}, J=13.9 \mathrm{~Hz}, 1 \mathrm{H}), 3.84$ (s, 3H), 3.70 (s, 3H), 1.93 $(\mathrm{s}, 3 \mathrm{H}) .{ }^{13} \mathrm{C}$ NMR $\left(101 \mathrm{MHz}, \mathrm{CDCl}_{3}\right) \delta[\mathrm{ppm}]=171.3,161.5,158.9,137.8,137.2,135.4,131.7$, 130.0, 128.6, 128.2, 128.0, 127.9, 127.6, 127.2, 119.8, 104.5, 98.7, 55.4, 55.3, 49.8, 22.0.

IR (KBr) v (cm $\left.{ }^{-1}\right)$ 3061, 3027, 2936, 1650, 1503, 1387, 1246, 1030, 979, 754, 696.

HRMS (ESI) m/z: $[\mathrm{M}+\mathrm{H}]^{+}$Calcd for $\mathrm{C}_{25} \mathrm{H}_{26} \mathrm{NO}_{3}$ 388.1907; found 388.1922 . 
<smiles>COc1cc(/C(=C/c2ccccc2)N(Cc2ccccc2)C(C)=O)cc(OC)c1OC</smiles>

$3 g$

(Z)-N-benzyl- $N$-(2-phenyl-1-(3,4,5-trimethoxyphenyl)vinyl)acetamide (3g)

Following the general procedure (eluent: petroleum ether/ethyl acetate $=10 / 1$ ), the desired product 3g was obtained as a pale yellow oil $\left(103.2 \mathrm{mg}, 91 \%\right.$ yield); ${ }^{1} \mathbf{H}$ NMR $\left(400 \mathrm{MHz}, \mathrm{CDCl}_{3}\right) \delta[\mathrm{ppm}]$ $=7.50-7.04(\mathrm{~m}, 10 \mathrm{H}), 6.76(\mathrm{~s}, 1 \mathrm{H}), 6.38(\mathrm{~s}, 2 \mathrm{H}), 5.07(\mathrm{~d}, J=13.7 \mathrm{~Hz}, 1 \mathrm{H}), 4.23(\mathrm{~d}, J=13.7 \mathrm{~Hz}$, 1H), $3.82(\mathrm{~s}, 3 \mathrm{H}), 3.65(\mathrm{~s}, 6 \mathrm{H}), 1.89(\mathrm{~s}, 3 \mathrm{H}) .{ }^{13} \mathbf{C} \mathbf{N M R}\left(101 \mathrm{MHz}, \mathrm{CDCl}_{3}\right) \delta[\mathrm{ppm}]=171.4,153.2$, $140.0,138.3,137.1,134.6,134.2,130.3,128.9,128.4,128.4,128.2,127.7,124.9,103.2,60.8$, $55.9,50.9,22.5$. This compound is known. ${ }^{[3]}$<smiles>CC(=O)N(Cc1ccccc1)/C(=C\c1ccc2c(c1)OCO2)c1ccccc1</smiles>

$3 \mathrm{~h}$

(Z)- $N$-(1-(benzo[d][1,3]dioxol-5-yl)-2-phenylvinyl)- $N$-benzylacetamide (3h)

Following the general procedure (eluent: petroleum ether/ethyl acetate $=10 / 1$ ), the desired product 3h was obtained as a pale yellow oil ( $80.4 \mathrm{mg}, 80 \%$ yield); ${ }^{1} \mathbf{H}$ NMR $\left(400 \mathrm{MHz}, \mathrm{CDCl}_{3}\right) \delta$ [ppm] $=7.36-7.28(\mathrm{~m}, 2 \mathrm{H}), 7.25-7.16(\mathrm{~m}, 6 \mathrm{H}), 7.13(\mathrm{dd}, J=7.6,2.1 \mathrm{~Hz}, 2 \mathrm{H}), 6.81-6.74(\mathrm{~m}, 2 \mathrm{H})$, $6.73(\mathrm{~d}, J=8.6 \mathrm{~Hz}, 1 \mathrm{H}), 6.67(\mathrm{~s}, 1 \mathrm{H}), 5.97(\mathrm{~s}, 2 \mathrm{H}), 4.70(\mathrm{~d}, J=13.7 \mathrm{~Hz}, 1 \mathrm{H}), 4.62(\mathrm{~d}, J=13.7 \mathrm{~Hz}$, $1 \mathrm{H}), 1.90(\mathrm{~s}, 3 \mathrm{H}) .{ }^{13} \mathbf{C}$ NMR $\left(101 \mathrm{MHz}, \mathrm{CDCl}_{3}\right) \delta[\mathrm{ppm}]=171.0,148.2,148.1,139.0,136.6$, 134.7, 132.4, 130.3, 128.7, 128.2, 128.1, 127.7, 125.5, 120.4, 108.3, 106.4, 101.4, 50.3, 22.0.

IR (KBr) v (cm-1) 3067, 3025, 2934, 1652, 1495, 1383, 1241, 934, 758, 708.

HRMS (ESI) m/z: $[\mathrm{M}+\mathrm{H}]^{+}$Calcd for $\mathrm{C}_{24} \mathrm{H}_{22} \mathrm{NO}_{3} 372.1594$; found 372.1612 . 
<smiles>CC(=O)N(Cc1ccccc1)/C(=C\c1ccccc1)c1ccc(F)cc1</smiles>

$3 \mathbf{i}$

(Z)- $N$-benzyl- $N$-(1-(4-fluorophenyl)-2-phenylvinyl)acetamide (3i)

Following the general procedure (eluent: petroleum ether/ethyl acetate $=18 / 1$ ), the desired product 3i was obtained as a pale yellow oil (85.4 mg, 92\% yield); ${ }^{1} \mathbf{H} \mathbf{~ N M R ~}\left(400 \mathrm{MHz}, \mathrm{CDCl}_{3}\right) \delta[\mathrm{ppm}]=$ $7.32-7.23(\mathrm{~m}, 7 \mathrm{H}), 7.21-7.17(\mathrm{~m}, 5 \mathrm{H}), 6.99(\mathrm{t}, J=8.6 \mathrm{~Hz}, 2 \mathrm{H}), 6.75(\mathrm{~s}, 1 \mathrm{H}), 4.71(\mathrm{~d}, J=13.7$ $\mathrm{Hz}, 1 \mathrm{H}), 4.64(\mathrm{~d}, J=13.7 \mathrm{~Hz}, 1 \mathrm{H}), 1.90(\mathrm{~s}, 3 \mathrm{H}) .{ }^{13} \mathbf{C} \mathbf{N M R}\left(101 \mathrm{MHz}, \mathrm{CDCl}_{3}\right) \delta[\mathrm{ppm}]=171.0$, $162.9\left(\mathrm{~d},{ }^{1} J_{C-F}=249.2 \mathrm{~Hz}\right), 138.4,136.6,134.5,134.2\left(\mathrm{~d},{ }^{4} J_{C-F}=3.1 \mathrm{~Hz}\right), 130.3,128.9,128.5$, $128.3,128.3,128.0,127.9\left(\mathrm{~d},{ }^{3} J_{C-F}=8.2 \mathrm{~Hz}\right), 127.8,126.6\left(\mathrm{~d},{ }^{5} J_{C-F}=1.5 \mathrm{~Hz}\right), 115.8\left(\mathrm{~d},{ }^{2} J_{C-F}=\right.$ $21.7 \mathrm{~Hz}), 50.3,22.0$. This compound is known. ${ }^{[3]}$<smiles>CC(=O)N(Cc1ccccc1)/C(=C\c1ccccc1)c1ccc(Cl)cc1</smiles>

3j

(Z)- $N$-benzyl- $N$-(1-(4-chlorophenyl)-2-phenylvinyl)acetamide (3j)

Following the general procedure (eluent: petroleum ether/ethyl acetate $=10 / 1$ ), the desired product 3j was obtained as a pale yellow oil (97.2 $\mathrm{mg}, 99 \%$ yield); ${ }^{1} \mathbf{H} \mathbf{~ N M R}\left(400 \mathrm{MHz}, \mathrm{CDCl}_{3}\right) \delta[\mathrm{ppm}]=$ $7.30-7.24(\mathrm{~m}, 7 \mathrm{H}), 7.22-7.17(\mathrm{~m}, 7 \mathrm{H}), 6.80(\mathrm{~s}, 1 \mathrm{H}), 4.71(\mathrm{~d}, J=13.7 \mathrm{~Hz}, 1 \mathrm{H}), 4.62(\mathrm{~d}, J=13.7$ $\mathrm{Hz}, 1 \mathrm{H}), 1.89$ (s, 3H). ${ }^{13} \mathbf{C}$ NMR (101 MHz, $\left.\mathrm{CDCl}_{3}\right) \delta[\mathrm{ppm}]=171.0,138.3,136.7,136.5,134.5$, 134.4, 130.3, 129.0, 128.9, 128.6, 128.4, 128.3, 127.8, 127.4, 127.1, 50.4, 22.1.

IR (KBr) v (cm-1) 3062, 3029, 2928, 1662, 1492, 1387, 1242, 975, 755, 696.

HRMS (ESI) m/z: $[\mathrm{M}+\mathrm{H}]^{+}$Calcd for $\mathrm{C}_{23} \mathrm{H}_{21} \mathrm{ClNO} 362.1306$; found 362.1323 . 
<smiles>CC(=O)N(Cc1ccccc1)/C(=C\c1ccccc1)c1ccc(Br)cc1</smiles>

$3 \mathbf{k}$

(Z)-N-benzyl- $N$-(1-(4-bromophenyl)-2-phenylvinyl)acetamide (3k)

Following the general procedure (eluent: petroleum ether/ethyl acetate $=10 / 1$ ), the desired product 3k was obtained as a pale yellow oil (92.2 $\mathrm{mg}, 84 \%$ yield); ${ }^{1} \mathbf{H}$ NMR (400 $\left.\mathrm{MHz}, \mathrm{CDCl}_{3}\right) \delta[\mathrm{ppm}]$ $=7.44-7.40(\mathrm{~m}, 2 \mathrm{H}), 7.30-7.23(\mathrm{~m}, 5 \mathrm{H}), 7.19(\mathrm{td}, J=4.9,4.3,2.6 \mathrm{~Hz}, 5 \mathrm{H}), 7.13(\mathrm{~d}, J=8.7 \mathrm{~Hz}$, 2H), $6.81(\mathrm{~s}, 1 \mathrm{H}), 4.70(\mathrm{~d}, J=13.7 \mathrm{~Hz}, 1 \mathrm{H}), 4.62(\mathrm{~d}, J=13.7 \mathrm{~Hz}, 1 \mathrm{H}), 1.89(\mathrm{~s}, 3 \mathrm{H}) .{ }^{13} \mathbf{C}$ NMR $\left(101 \mathrm{MHz}, \mathrm{CDCl}_{3}\right) \delta[\mathrm{ppm}]=170.9,138.3,137.1,136.4,134.3,131.8,130.2,128.8,128.6,128.3$, $128.2,127.8,127.6,127.1,122.6,50.3,22.0$.

IR (KBr) v (cm $\left.{ }^{-1}\right)$ 3061, 3029, 2928, 1661, 1491, 1387, 1242, 975, 754, 696.

HRMS (ESI) m/z: $[\mathrm{M}+\mathrm{H}]^{+}$Calcd for $\mathrm{C}_{23} \mathrm{H}_{21} \mathrm{BrNO}$ 406.0801; found 406.0819.<smiles>CC(=O)N(Cc1ccccc1)/C(=C\c1ccccc1)c1cccc(Br)c1</smiles>

31

(Z)-N-benzyl- $N$-(1-(3-bromophenyl)-2-phenylvinyl)acetamide (3I)

Following the general procedure (eluent: petroleum ether/ethyl acetate $=18 / 1$ ), the desired product 3I was obtained as a pale yellow oil $(106.5 \mathrm{mg}, 97 \%$ yield $) ;{ }^{1} \mathbf{H}$ NMR $\quad\left(400 \mathrm{MHz}, \mathrm{CDCl}_{3}\right) \delta[\mathrm{ppm}]$ $=7.40(\mathrm{dd}, J=4.6,2.3 \mathrm{~Hz}, 2 \mathrm{H}), 7.27(\mathrm{dt}, J=12.6,2.6 \mathrm{~Hz}, 5 \mathrm{H}), 7.22-7.12(\mathrm{~m}, 7 \mathrm{H}), 6.81(\mathrm{~s}, 1 \mathrm{H})$, $4.72(\mathrm{~d}, J=13.7 \mathrm{~Hz}, 1 \mathrm{H}), 4.61(\mathrm{~d}, J=13.7 \mathrm{~Hz}, 1 \mathrm{H}), 1.89(\mathrm{~s}, 3 \mathrm{H}) .{ }^{13} \mathbf{C} \mathbf{N M R}\left(101 \mathrm{MHz}, \mathrm{CDCl}_{3}\right) \delta$ $[\mathrm{ppm}]=170.8,140.2,137.8,136.3,134.1,131.4,130.2,130.1,129.0,128.8,128.7,128.3,128.2$, $127.8,124.5,123.0,50.3,22.0$.

IR (KBr) v (cm-1) 3061, 3028, 2927, 1656, 1493, 1386, 1235, 975, 755, 694.

HRMS (ESI) m/z: $[\mathrm{M}+\mathrm{H}]^{+}$Calcd for $\mathrm{C}_{23} \mathrm{H}_{21} \mathrm{BrNO}$ 406.0801; found 406.0801 . 
<smiles>CC(=O)N(Cc1ccccc1)/C(=C\c1ccccc1)c1ccccc1Br</smiles>

$3 m$

(Z) -N-benzyl- $N$-(1-(2-bromophenyl)-2-phenylvinyl)acetamide (3m)

Following the general procedure (eluent: petroleum ether/ethyl acetate $=20 / 1$ ), the desired product 3m was obtained as a pale yellow oil $\left(98.7 \mathrm{mg}, 90 \%\right.$ yield); ${ }^{1} \mathbf{H}$ NMR $\left(400 \mathrm{MHz}, \mathrm{CDCl}_{3}\right) \delta[\mathrm{ppm}]$ $=7.65(\mathrm{dd}, J=8.0,1.2 \mathrm{~Hz}, 1 \mathrm{H}), 7.43-7.36(\mathrm{~m}, 2 \mathrm{H}), 7.32-7.27(\mathrm{~m}, 2 \mathrm{H}), 7.24(\mathrm{~m}, 3 \mathrm{H}), 7.21-$ $7.16(\mathrm{~m}, 4 \mathrm{H}), 7.05-6.97$ (m, 2H), $6.61(\mathrm{~s}, 1 \mathrm{H}), 4.99(\mathrm{~s}, 1 \mathrm{H}), 4.18(\mathrm{~s}, 1 \mathrm{H}), 1.93(\mathrm{~s}, 3 \mathrm{H}) .{ }^{13} \mathbf{C}$ NMR $\left(101 \mathrm{MHz}, \mathrm{CDCl}_{3}\right) \delta[\mathrm{ppm}]=171.0,137.9,137.3,136.6,134.6,134.3,131.1,131.1,130.1,130.0$, $129.9,128.7,128.3,128.3,127.9,127.6,122.6,49.7,22.2$.

IR (KBr) v (cm-1) 3061, 3028, 2932, 1656, 1492, 1385,1245, 978, 754, 695.

HRMS (ESI) m/z: $[\mathrm{M}+\mathrm{H}]^{+}$Calcd for $\mathrm{C}_{23} \mathrm{H}_{21} \mathrm{BrNO} 406.0801$; found 406.0819 .<smiles>CC(=O)N(Cc1ccccc1)/C(=C\c1ccccc1)c1ccc(C(F)(F)F)cc1</smiles>

$3 n$

(Z)- $N$-benzyl- $N$-(2-phenyl-1-(4-(trifluoromethyl)phenyl)vinyl)acetamide (3n)

Following the general procedure (eluent: petroleum ether/ethyl acetate $=20 / 1$ ), the desired product 3n was obtained as a pale yellow oil (79.8 $\mathrm{mg}, 75 \%$ yield); ${ }^{1} \mathbf{H}$ NMR $\left(400 \mathrm{MHz}, \mathrm{CDCl}_{3}\right) \delta[\mathrm{ppm}]$ $=7.55(\mathrm{~d}, J=8.2 \mathrm{~Hz}, 2 \mathrm{H}), 7.40-7.35(\mathrm{~m}, 2 \mathrm{H}), 7.28(\mathrm{dt}, J=4.0,1.9 \mathrm{~Hz}, 5 \mathrm{H}), 7.25(\mathrm{~d}, J=3.1 \mathrm{~Hz}$, 2H), $7.19(\mathrm{dd}, J=5.0,1.9 \mathrm{~Hz}, 3 \mathrm{H}), 6.91(\mathrm{~s}, 1 \mathrm{H}), 4.74(\mathrm{~d}, J=13.7 \mathrm{~Hz}, 1 \mathrm{H}), 4.63(\mathrm{~d}, J=13.8 \mathrm{~Hz}$, 1H), $1.90(\mathrm{~s}, 3 \mathrm{H}) .{ }^{13} \mathrm{C}$ NMR $\left(101 \mathrm{MHz}, \mathrm{CDCl}_{3}\right) \delta[\mathrm{ppm}]=170.8,141.7,138.0,136.3,134.0$, $130.3\left(\mathrm{~d},{ }^{2} J_{C-F}=32.3 \mathrm{~Hz}\right), 130.2,128.9,128.9,128.7,128.5,128.3,127.8,126.3,125.6\left(\mathrm{q},{ }^{3} J_{C-F}=\right.$ $3.8 \mathrm{~Hz}), 123.9\left(\mathrm{~d},{ }^{1} J_{C-F}=272.2 \mathrm{~Hz}\right), 50.4,22.0$.

IR (KBr) v (cm-1) 3063, 3029, 2931, 1664, 1493, 1388, 1243, 975, 755, 696.

HRMS (ESI) m/z: $[\mathrm{M}+\mathrm{H}]^{+}$Calcd for $\mathrm{C}_{24} \mathrm{H}_{21} \mathrm{~F}_{3} \mathrm{NO}$ 396.1570; found 396.1587 . 
<smiles>CC(=O)N(Cc1ccccc1)C(=Cc1ccccc1)c1ccc(C#N)cc1</smiles>

30

(Z)- $N$-benzyl- $N$-(1-(4-cyanophenyl)-2-phenylvinyl)acetamide (3o)

Following the general procedure (eluent: petroleum ether/ethyl acetate $=15 / 1$ ), the desired product 30 was obtained as a pale yellow oil $\left(63.2 \mathrm{mg}, 66 \%\right.$ yield); $\mathrm{R}_{f}=0.38$ (Petroleum ether/EtOAc $=$ 10/1). ${ }^{1} \mathbf{H}$ NMR $\left(400 \mathrm{MHz}, \mathrm{CDCl}_{3}\right) \delta[\mathrm{ppm}]=7.57-7.49(\mathrm{~m}, 2 \mathrm{H}), 7.34-7.26(\mathrm{~m}, 7 \mathrm{H}), 7.24(\mathrm{dd}$, $J=7.4,2.2 \mathrm{~Hz}, 2 \mathrm{H}), 7.17$ (tt, $J=4.2,2.0 \mathrm{~Hz}, 3 \mathrm{H}), 6.93(\mathrm{~s}, 1 \mathrm{H}), 4.83$ (d, $J=13.7 \mathrm{~Hz}, 1 \mathrm{H}), 4.50$ (d, $J=13.8 \mathrm{~Hz}, 1 \mathrm{H}), 1.87(\mathrm{~s}, 3 \mathrm{H}) .{ }^{13} \mathbf{C} \mathbf{~ N M R}\left(101 \mathrm{MHz}, \mathrm{CDCl}_{3}\right) \delta[\mathrm{ppm}]=170.6,142.6,137.5,136.1$, $133.7,132.3,130.1,129.5,129.2,128.9,128.5,128.2,127.9,126.3,118.3,111.7,50.3,21.9$.

IR (KBr) v (cm-1) 3062, 3030, 2929, 2226, 1663, 1500, 1388, 1242, 975, 754, 696.

HRMS (ESI) m/z: [M+H] $]^{+}$Calcd for $\mathrm{C}_{24} \mathrm{H}_{21} \mathrm{~N}_{2} \mathrm{O}$ 353.1648; found 353.1663 .<smiles>CC(=O)N(Cc1ccccc1)/C(=C\c1ccccc1)c1ccc([N+](=O)[O-])cc1</smiles>

$3 p$

(Z)- $N$-benzyl- $N$-(1-(4-nitrophenyl)-2-phenylvinyl)acetamide (3p)

Following the general procedure (eluent: petroleum ether/ethyl acetate $=10 / 1$ ), the desired product 3p was obtained as a yellow oil $(81.5 \mathrm{mg}, 81 \%$ yield $) ;{ }^{1} \mathbf{H}$ NMR $\left(400 \mathrm{MHz}, \mathrm{CDCl}_{3}\right) \delta[\mathrm{ppm}]=$ $8.10(\mathrm{~d}, J=8.9 \mathrm{~Hz}, 2 \mathrm{H}), 7.37$ (d, $J=8.9 \mathrm{~Hz}, 2 \mathrm{H}), 7.32$ (t, $J=2.7 \mathrm{~Hz}, 5 \mathrm{H}), 7.25$ (dd, $J=7.4,2.1$ Hz, 2H), $7.21-7.13$ (m, 3H), 7.00 (s, 1H), 4.88 (d, $J=13.8 \mathrm{~Hz}, 1 \mathrm{H}), 4.50$ (d, $J=13.8 \mathrm{~Hz}, 1 \mathrm{H})$, $1.89(\mathrm{~s}, 3 \mathrm{H}) .{ }^{13} \mathrm{C}$ NMR $\left(101 \mathrm{MHz}, \mathrm{CDCl}_{3}\right) \delta[\mathrm{ppm}]=170.7,147.3,144.6,137.3,136.1,133.6$, 130.2, 130.1, 129.5, 129.1, 128.7, 128.4, 128.0, 126.5, 123.9, 50.4, 22.1.

IR (KBr) v (cm-1) 3064, 3029, 2932, 1653, 1493, 1391, 1342, 1248, 975, 754, 698.

HRMS (ESI) m/z: [M+H] $]^{+}$Calcd for $\mathrm{C}_{23} \mathrm{H}_{21} \mathrm{~N}_{2} \mathrm{O}_{3}$ 373.1547; found 373.1562. 
<smiles>CC(=O)N(Cc1ccccc1)C(=Cc1ccccc1)c1ccc(-c2ccccc2)cc1</smiles>

$3 q$

(Z)- $N$-(1-([1,1'-biphenyl]-4-yl)-2-phenylvinyl)- $N$-benzylacetamide (3q)

Following the general procedure (eluent: petroleum ether/ethyl acetate $=20 / 1$ ), the desired product 3q was obtained as a pale yellow oil (105.7 mg, 97\% yield); ${ }^{1} \mathbf{H}$ NMR $\left(400 \mathrm{MHz}, \mathrm{CDCl}_{3}\right) \delta[\mathrm{ppm}]$ $=7.66-7.55(\mathrm{~m}, 4 \mathrm{H}), 7.48(\mathrm{t}, J=7.6 \mathrm{~Hz}, 2 \mathrm{H}), 7.42-7.33(\mathrm{~m}, 5 \mathrm{H}), 7.30-7.18(\mathrm{~m}, 8 \mathrm{H}), 6.90(\mathrm{~s}$, 1H), $4.79(\mathrm{~d}, J=13.7 \mathrm{~Hz}, 1 \mathrm{H}), 4.70(\mathrm{~d}, J=13.7 \mathrm{~Hz}, 1 \mathrm{H}), 1.96(\mathrm{~s}, 3 \mathrm{H}) .{ }^{13} \mathbf{C}$ NMR $(101 \mathrm{MHz}$, $\left.\mathrm{CDCl}_{3}\right) \delta[\mathrm{ppm}]=171.1,141.3,140.1,138.8,136.8,136.5,134.5,130.3,128.8,128.8,128.4$, $128.3,128.2,127.7,127.6,127.4,126.9,126.7,126.5,50.4,22.1$. This compound is known. ${ }^{[3]}$<smiles>CC(=O)N(Cc1ccccc1)/C(C)=C\c1ccccc1</smiles>

(Z)-N-benzyl- $N$-(1-phenylprop-1-en-2-yl)acetamide (3r)

Following the general procedure (eluent: petroleum ether/ethyl acetate $=15 / 1$ ), the desired product 3r was obtained as a pale yellow oil (32.8 $\mathrm{mg}, 46 \%$ yield); ${ }^{1} \mathbf{H}$ NMR $\left(400 \mathrm{MHz}, \mathrm{CDCl}_{3}\right) \delta[\mathrm{ppm}]$ $=7.31(\mathrm{dd}, J=6.9,1.8 \mathrm{~Hz}, 3 \mathrm{H}), 7.29-7.19(\mathrm{~m}, 7 \mathrm{H}), 6.25(\mathrm{~s}, 1 \mathrm{H}), 5.33(\mathrm{~d}, J=14.3 \mathrm{~Hz}, 1 \mathrm{H}), 4.02$ $(\mathrm{d}, J=14.3 \mathrm{~Hz}, 1 \mathrm{H}), 2.05(\mathrm{~s}, 3 \mathrm{H}), 1.79(\mathrm{~d}, J=1.3 \mathrm{~Hz}, 3 \mathrm{H}) .{ }^{13} \mathbf{C} \mathbf{N M R}\left(101 \mathrm{MHz}, \mathrm{CDCl}_{3}\right) \delta[\mathrm{ppm}]$ $=169.8,137.5,137.2,134.4,128.9,128.8,128.3,127.8,127.7,127.4,126.8,49.5,24.4,21.6$. This compound is known. ${ }^{[8]}$<smiles>CC(=O)N(Cc1ccc(C)cc1)/C(=C\c1ccccc1)c1ccccc1</smiles>

3s

(Z)- $N$-(1,2-diphenylvinyl)- $N$-(4-methylbenzyl)acetamide (3s) 
Following the general procedure (eluent: petroleum ether/ethyl acetate $=20 / 1$ ), the desired product 3s was obtained as a pale yellow oil ( $82.2 \mathrm{mg}, 89 \%$ yield $)$; ${ }^{1} \mathbf{H} \mathbf{~ N M R}\left(400 \mathrm{MHz}, \mathrm{CDCl}_{3}\right) \delta[\mathrm{ppm}]=$ 7.34 (s, 5H), $7.26-7.13(\mathrm{~m}, 7 \mathrm{H}), 7.00(\mathrm{~d}, J=7.7 \mathrm{~Hz}, 2 \mathrm{H}), 6.81(\mathrm{~s}, 1 \mathrm{H}), 4.79(\mathrm{~d}, J=13.7 \mathrm{~Hz}, 1 \mathrm{H})$, $4.52(\mathrm{~d}, J=13.7 \mathrm{~Hz}, 1 \mathrm{H}), 2.27(\mathrm{~s}, 3 \mathrm{H}), 1.90(\mathrm{~s}, 3 \mathrm{H}) .{ }^{13} \mathbf{C} \mathbf{N M R}\left(101 \mathrm{MHz}, \mathrm{CDCl}_{3}\right) \delta[\mathrm{ppm}]=$ $170.9,139.2,138.0,137.2,134.6,133.4,130.2,128.8,128.7,128.7,128.5,128.2,128.2,126.8$, 126.1, 50.0, 22.0, 21.0.

IR (KBr) v (cm (c) $\left.^{-1}\right) 3055,3025,2926,1660,1493,1387,1240,1030,974,768,695$.

HRMS (ESI) m/z: $[\mathrm{M}+\mathrm{H}]^{+}$Calcd for $\mathrm{C}_{24} \mathrm{H}_{24} \mathrm{NO} 342.1852$; found 342.1858 .<smiles>CC(=O)N(Cc1ccc(F)cc1)/C(=C\c1ccccc1)c1ccccc1</smiles>

(Z)-N-(1,2-diphenylvinyl)- $N$-(4-fluorobenzyl)acetamide (3t)

Following the general procedure (eluent: petroleum ether/ethyl acetate $=20 / 1$ ), the desired product 3t was obtained as a pale yellow oil ( $80.7 \mathrm{mg}, 87 \%$ yield); ${ }^{1} \mathbf{H} \mathbf{N M R}\left(400 \mathrm{MHz}, \mathrm{CDCl}_{3}\right) \delta[\mathrm{ppm}]=$ $7.33(\mathrm{t}, J=2.7 \mathrm{~Hz}, 5 \mathrm{H}), 7.30-7.22(\mathrm{~m}, 5 \mathrm{H}), 7.17(\mathrm{dd}, J=7.4,2.4 \mathrm{~Hz}, 2 \mathrm{H}), 6.90-6.79(\mathrm{~m}, 3 \mathrm{H})$, $4.75(\mathrm{~d}, J=13.8 \mathrm{~Hz}, 1 \mathrm{H}), 4.55(\mathrm{~d}, J=13.8 \mathrm{~Hz}, 1 \mathrm{H}), 1.91(\mathrm{~s}, 3 \mathrm{H}) .{ }^{13} \mathbf{C}$ NMR $\left(101 \mathrm{MHz}, \mathrm{CDCl}_{3}\right) \delta$ $[\mathrm{ppm}]=171.0,162.2\left(\mathrm{~d},{ }^{1} J_{C-F}=246.3 \mathrm{~Hz}\right), 138.9,137.7,134.4,132.4(\mathrm{~d}, J=3.3 \mathrm{~Hz}), 131.9(\mathrm{~d}$, $\left.{ }^{3} J_{C-F}=8.2 \mathrm{~Hz}\right), 128.8,128.7,128.7,128.4,128.2,126.9,126.0,114.9\left(\mathrm{~d},{ }^{2} J_{C-F}=21.2 \mathrm{~Hz}\right), 49.5$, 21.9.

IR (KBr) v (cm-1) 3057, 3026, 2934, 1657, 1509, 1388, 1223, 1031, 977, 769, 695.

HRMS (ESI) m/z: $[\mathrm{M}+\mathrm{H}]^{+}$Calcd for $\mathrm{C}_{23} \mathrm{H}_{21} \mathrm{FNO} 346.1602$; found 346.1608 .<smiles>CC(=O)N(C)/C(=C\c1ccccc1)c1ccccc1</smiles>

$3 u$

(Z)- $N$-(1,2-diphenylvinyl)- $N$-methylacetamide (3u)

Following the general procedure (eluent: petroleum ether/ethyl acetate $=20 / 1$ ), the desired product 3u was obtained as a pale yellow oil (61.0 mg, $90 \%$ yield); ${ }^{1} \mathbf{H}$ NMR $\left(400 \mathrm{MHz}, \mathrm{CDCl}_{3}\right) \delta[\mathrm{ppm}]$ 
$=7.51-7.27(\mathrm{~m}, 10 \mathrm{H}), 6.88(\mathrm{~s}, 1 \mathrm{H}), 3.11(\mathrm{~s}, 3 \mathrm{H}), 1.87(\mathrm{~s}, 3 \mathrm{H}) .{ }^{13} \mathbf{C}$ NMR $\left(101 \mathrm{MHz}, \mathrm{CDCl}_{3}\right) \delta$ $[\mathrm{ppm}]=171.0,140.2,137.1,134.6,129.0,129.0,128.8,128.4,128.3,125.6,125.3,34.1,21.3$. This compound is known. ${ }^{[9]}$<smiles>CCC(=O)N(Cc1ccccc1)/C(=C\c1ccccc1)c1ccccc1</smiles>

(Z)-N-benzyl- $N$-(1,2-diphenylvinyl)propionamide (3v)

Following the general procedure (eluent: petroleum ether/ethyl acetate $=20 / 1$ ), the desired product 3v was obtained as a pale yellow oil $\left(79.7 \mathrm{mg}, 86 \%\right.$ yield); ${ }^{1} \mathbf{H}$ NMR $\left(400 \mathrm{MHz}, \mathrm{CDCl}_{3}\right) \delta[\mathrm{ppm}]$ $=7.40-7.30(\mathrm{~m}, 7 \mathrm{H}), 7.27-7.16(\mathrm{~m}, 8 \mathrm{H}), 6.86(\mathrm{~s}, 1 \mathrm{H}), 4.77(\mathrm{~d}, J=13.7 \mathrm{~Hz}, 1 \mathrm{H}), 4.67(\mathrm{~d}, J=$ $13.7 \mathrm{~Hz}, 1 \mathrm{H}), 2.21$ (p, $J=7.5 \mathrm{~Hz}, 2 \mathrm{H}), 0.99$ (t, $J=7.4 \mathrm{~Hz}, 3 \mathrm{H}) .{ }^{13} \mathbf{C}$ NMR $\left(101 \mathrm{MHz}, \mathrm{CDCl}_{3}\right) \delta$ $[\mathrm{ppm}]=174.3,138.7,138.0,136.6,134.4,130.2,128.6,128.6,128.4,128.2,128.2,128.1,127.5$, $126.6,126.0,50.5,26.7,9.2$.

IR (KBr) v (cm-1) 3060, 3028, 2936, 1659, 1494, 1392, 1224, 1071, 970, 763, 696.

HRMS (ESI) m/z: $[\mathrm{M}+\mathrm{H}]^{+}$Calcd for $\mathrm{C}_{24} \mathrm{H}_{24} \mathrm{NO} 342.1852$; found 342.1861 .<smiles>CC(=O)N(Cc1ccccc1)C(=Cc1ccc(C)cc1)c1ccccc1</smiles>

(Z)-N-benzyl- $N$-(1-phenyl-2-(p-tolyl)vinyl)acetamide (4b)

Following the general procedure (eluent: petroleum ether/ethyl acetate $=20 / 1$ ), the desired product 4b was obtained as a pale yellow oil (91.7 mg, 99\% yield); ${ }^{1} \mathbf{H}$ NMR (400 MHz, $\left.\mathrm{CDCl}_{3}\right) \delta[\mathrm{ppm}]$ $=7.37-7.30(\mathrm{~m}, 7 \mathrm{H}), 7.22-7.17(\mathrm{~m}, 3 \mathrm{H}), 7.08(\mathrm{q}, J=8.2 \mathrm{~Hz}, 4 \mathrm{H}), 6.81(\mathrm{~s}, 1 \mathrm{H}), 4.81(\mathrm{~d}, J=$ $13.7 \mathrm{~Hz}, 1 \mathrm{H}), 4.62(\mathrm{~d}, J=13.7 \mathrm{~Hz}, 1 \mathrm{H}), 2.32(\mathrm{~s}, 3 \mathrm{H}), 1.93(\mathrm{~s}, 3 \mathrm{H}) .{ }^{13} \mathbf{C} \mathbf{N M R}\left(101 \mathrm{MHz}, \mathrm{CDCl}_{3}\right) \delta$ $[\mathrm{ppm}]=171.1,138.5,138.4,138.2,136.7,131.9,130.4,129.5,128.8,128.5,128.3,128.2,127.7$, $127.0,126.1,50.4,22.1,21.3$. This compound is known. ${ }^{[8]}$ 
<smiles>CC(=O)N(Cc1ccccc1)C(=Cc1cccc(C)c1)c1ccccc1</smiles>

(Z)- $N$-benzyl- $N$-(1-phenyl-2-(m-tolyl)vinyl)acetamide (4c)

Following the general procedure (eluent: petroleum ether/ethyl acetate $=15 / 1$ ), the desired product $4 \mathbf{c}$ was obtained as a pale yellow oil $\left(84.5 \mathrm{mg}, 92 \%\right.$ yield); ${ }^{1} \mathbf{H}$ NMR $\left(400 \mathrm{MHz}, \mathrm{CDCl}_{3}\right) \delta[\mathrm{ppm}]$ $=7.39-7.31(\mathrm{~m}, 7 \mathrm{H}), 7.23-7.19(\mathrm{~m}, 3 \mathrm{H}), 7.13(\mathrm{t}, J=7.6 \mathrm{~Hz}, 1 \mathrm{H}), 7.00(\mathrm{dd}, J=18.6,7.6 \mathrm{~Hz}$, 2H), 6.90 (s, 1H), 6.79 (s, 1H), 4.89 (d, $J=13.7 \mathrm{~Hz}, 1 \mathrm{H}), 4.46$ (d, $J=13.7 \mathrm{~Hz}, 1 \mathrm{H}), 2.21(\mathrm{~s}, 3 \mathrm{H})$, $1.91(\mathrm{~s}, 3 \mathrm{H}) .{ }^{13} \mathrm{C}$ NMR $\left(101 \mathrm{MHz}, \mathrm{CDCl}_{3}\right) \delta[\mathrm{ppm}]=171.0,139.0,138.3,138.1,136.7,134.6$, $130.4,129.2,128.9,128.8,128.6,128.3,127.7,127.4,126.2,125.5,50.3,22.0,21.4$.

IR (KBr) v (cm-1) 3060, 3029, 2928, 1660, 1493, 1388, 1245, 975, 750, 697.

HRMS (ESI) m/z: [M+H] $]^{+}$Calcd for $\mathrm{C}_{24} \mathrm{H}_{24} \mathrm{NO} 342.1852$; found 342.1865 .<smiles>CC(=O)N(Cc1ccccc1)/C(=C\c1ccccc1C)c1ccccc1</smiles>

(Z)-N-benzyl- $N$-(1-phenyl-2-(o-tolyl)vinyl)acetamide (4d)

Following the general procedure (eluent: petroleum ether/ethyl acetate $=15 / 1$ ), the desired product 4d was obtained as a pale yellow oil (90.6 mg, 98\% yield); ${ }^{1} \mathbf{H}$ NMR (400 MHz, CDCl3) $\delta$ [ppm] $=7.34(\mathrm{qd}, J=5.7,5.0,2.2 \mathrm{~Hz}, 7 \mathrm{H}), 7.26(\mathrm{q}, J=2.8 \mathrm{~Hz}, 3 \mathrm{H}), 7.23-7.14(\mathrm{~m}, 2 \mathrm{H}), 7.07-7.01(\mathrm{~m}$, 1H), 6.89 (d, $J=7.0 \mathrm{~Hz}, 2 \mathrm{H}), 4.70$ (d, $J=13.8 \mathrm{~Hz}, 1 \mathrm{H}), 4.56$ (d, $J=13.8 \mathrm{~Hz}, 1 \mathrm{H}), 2.35$ (s, 3H), $1.90(\mathrm{~s}, 3 \mathrm{H}) .{ }^{13} \mathbf{C}$ NMR $\left(101 \mathrm{MHz}, \mathrm{CDCl}_{3}\right) \delta[\mathrm{ppm}]=171.2,140.3,138.6,137.0,136.5,133.9$, $130.5,130.4,128.9,128.7,128.4,128.3,127.8,126.9,126.4,126.4,124.6,50.7,22.4,20.3$.

IR (KBr) v (cm-1) 3060, 3028, 2924, 1660, 1493, 1384, 1241, 975, 757, 697.

HRMS (ESI) m/z: $[\mathrm{M}+\mathrm{H}]^{+}$Calcd for $\mathrm{C}_{24} \mathrm{H}_{24} \mathrm{NO} 342.1852$; found 342.1868 . 
<smiles>CC(=O)N(Cc1ccccc1)C(=Cc1cc(C)cc(C)c1)c1ccccc1</smiles>

(Z)- $N$-benzyl- $N$-(2-(3,5-dimethylphenyl)-1-phenylvinyl)acetamide (4e)

Following the general procedure (eluent: petroleum ether/ethyl acetate $=15 / 1$ ), the desired product 4e was obtained as a pale yellow oil (91.9 mg, 96\% yield); $\left.{ }^{1} \mathbf{H} \mathbf{~ N M R ~ ( 4 0 0 ~ M H z , ~} \mathrm{CDCl}_{3}\right) \delta[\mathrm{ppm}]$ $=7.42-7.33(\mathrm{~m}, 7 \mathrm{H}), 7.22(\mathrm{dd}, J=5.0,2.0 \mathrm{~Hz}, 3 \mathrm{H}), 6.85(\mathrm{~d}, J=1.9 \mathrm{~Hz}, 1 \mathrm{H}), 6.79-6.71(\mathrm{~m}, 3 \mathrm{H})$, $4.99(\mathrm{~d}, J=13.7 \mathrm{~Hz}, 1 \mathrm{H}), 4.37(\mathrm{~d}, J=13.7 \mathrm{~Hz}, 1 \mathrm{H}), 2.19$ (s, 6H), $1.92(\mathrm{~s}, 3 \mathrm{H}) .{ }^{13} \mathbf{C}$ NMR $(101$ $\left.\mathrm{MHz}, \mathrm{CDCl}_{3}\right) \delta[\mathrm{ppm}]=171.1,138.6,138.1,138.1,136.7,134.5,130.5,130.2,128.9,128.6$, $128.4,127.7,127.7,126.2,126.1,50.3,22.0,21.2$.

IR (KBr) v (cm-1) 3060, 3028, 2920, 1659, 1492, 1389, 1245, 975, 762, 698.

HRMS (ESI) m/z: $[\mathrm{M}+\mathrm{H}]^{+}$Calcd for $\mathrm{C}_{25} \mathrm{H}_{26} \mathrm{NO} 356.2009$; found 356.2025 .<smiles>CC(=O)N(Cc1ccccc1)/C(=C\c1ccc(C(C)(C)C)cc1)c1ccccc1</smiles>

(Z)- $N$-benzyl- $N$-(2-(4-(tert-butyl)phenyl)-1-phenylvinyl)acetamide (4f)

Following the general procedure (eluent: petroleum ether/ethyl acetate $=20 / 1$ ), the desired product 4f was obtained as a pale yellow oil (87.0 mg, $84 \%$ yield); ${ }^{1} \mathbf{H} \mathbf{~ N M R ~}\left(400 \mathrm{MHz}, \mathrm{CDCl}_{3}\right) \delta[\mathrm{ppm}]=$ $7.33-7.29(\mathrm{~m}, 7 \mathrm{H}), 7.29-7.25(\mathrm{~m}, 2 \mathrm{H}), 7.20-7.12(\mathrm{~m}, 5 \mathrm{H}), 6.82(\mathrm{~s}, 1 \mathrm{H}), 4.74(\mathrm{~d}, J=13.7 \mathrm{~Hz}$, 1H), $4.66(\mathrm{~d}, J=13.8 \mathrm{~Hz}, 1 \mathrm{H}), 1.92(\mathrm{~s}, 3 \mathrm{H}), 1.31(\mathrm{~s}, 9 \mathrm{H}) .{ }^{13} \mathbf{C ~ N M R}\left(101 \mathrm{MHz}, \mathrm{CDCl}_{3}\right) \delta[\mathrm{ppm}]=$ $171.2,151.6,138.6,138.2,136.7,131.8,130.4,128.7,128.4,128.2,127.6,126.8,126.0,125.7$, $50.5,34.6,31.2,22.1$.

IR (KBr) v (cm-1) 3061, 3029, 2961, 1660, 1493, 1386, 1242, 974, 760, 697.

HRMS (ESI) m/z: $[\mathrm{M}+\mathrm{H}]^{+}$Calcd for $\mathrm{C}_{27} \mathrm{H}_{30} \mathrm{NO} 384.2322$; found 384.2340 . 
<smiles>CC(=O)N(Cc1ccccc1)C(=Cc1ccc(C)c(Cl)c1)c1ccccc1</smiles>

(Z)- $N$-benzyl- $N$-(2-(3-chloro-4-methylphenyl)-1-phenylvinyl)acetamide (4g)

Following the general procedure (eluent: petroleum ether/ethyl acetate $=15 / 1$ ), the desired product 4g was obtained as a pale yellow oil $(93,2 \mathrm{mg}, 92 \%) ;{ }^{1} \mathbf{H} \mathbf{~ N M R}\left(400 \mathrm{MHz}, \mathrm{CDCl}_{3}\right) \delta[\mathrm{ppm}]=7.41$ - $7.30(\mathrm{~m}, 7 \mathrm{H}), 7.26-7.21(\mathrm{~m}, 3 \mathrm{H}), 7.17-7.10(\mathrm{~m}, 1 \mathrm{H}), 7.05(\mathrm{dd}, J=8.1,4.1 \mathrm{~Hz}, 1 \mathrm{H}), 6.94-$ $6.87(\mathrm{~m}, 1 \mathrm{H}), 6.77-6.70(\mathrm{~m}, 1 \mathrm{H}), 4.97(\mathrm{~d}, J=13.7 \mathrm{~Hz}, 1 \mathrm{H}), 4.42$ (d, $J=13.7 \mathrm{~Hz}, 1 \mathrm{H}), 2.33$ (s, 3H), 1.93 (s, 3H). ${ }^{13} \mathbf{C}$ NMR (101 MHz, $\left.\mathrm{CDCl}_{3}\right) \delta[\mathrm{ppm}]=170.9,139.5,137.6,136.4,136.3$, $134.7,133.9,131.2,130.3,129.0,128.9,128.9,128.4,127.8,126.2,125.9,125.9,50.3,22.0,19.9$. IR (KBr) v (cm-1) 3053, 3027, 2929, 1648, 1494, 1389, 1242, 975, 758, 701.

HRMS (ESI) m/z: $[\mathrm{M}+\mathrm{H}]^{+}$Calcd for $\mathrm{C}_{24} \mathrm{H}_{22} \mathrm{ClNO} 376.1463$; found 376.1478 .<smiles>COc1ccc(/C=C(/c2ccccc2)N(Cc2ccccc2)C(C)=O)cc1</smiles>

(Z)-N-benzyl- $N$-(2-(4-methoxyphenyl)-1-phenylvinyl)acetamide (4h)

Following the general procedure (eluent: petroleum ether/ethyl acetate $=10 / 1$ ), the desired product 4h was obtained as a pale yellow oil (92.5 mg, 96\% yield); ${ }^{1} \mathbf{H}$ NMR (400 MHz, $\left.\mathrm{CDCl}_{3}\right) \delta$ [ppm] $=7.31(\mathrm{q}, J=2.3 \mathrm{~Hz}, 7 \mathrm{H}), 7.18(\mathrm{dd}, J=4.4,2.1 \mathrm{~Hz}, 3 \mathrm{H}), 7.13(\mathrm{~d}, J=8.8 \mathrm{~Hz}, 2 \mathrm{H}), 6.80-6.72(\mathrm{~m}$, 3H), $4.82(\mathrm{~d}, J=13.7 \mathrm{~Hz}, 1 \mathrm{H}), 4.56(\mathrm{~d}, J=13.7 \mathrm{~Hz}, 1 \mathrm{H}), 3.78(\mathrm{~s}, 3 \mathrm{H}), 1.92(\mathrm{~s}, 3 \mathrm{H}) .{ }^{13} \mathbf{C}$ NMR $\left(101 \mathrm{MHz}, \mathrm{CDCl}_{3}\right) \delta[\mathrm{ppm}]=171.3,159.6,138.1,137.2,136.6,130.4,129.8,128.8,128.3,128.2$, $127.7,127.3,126.6,125.9,114.2,55.2,50.3,22.0$. This compound is known. ${ }^{[4]}$ 
<smiles>COc1cccc(/C=C(\c2ccccc2)N(Cc2ccccc2)C(C)=O)c1</smiles>

(Z)- $N$-benzyl- $N$-(2-(3-methoxyphenyl)-1-phenylvinyl)acetamide (4i)

Following the general procedure (eluent: petroleum ether/ethyl acetate $=15 / 1$ ), the desired product 4i was obtained as a pale yellow oil (94.7 mg, 98\% yield); ${ }^{1} \mathbf{H} \mathbf{~ N M R ~}\left(400 \mathrm{MHz}, \mathrm{CDCl}_{3}\right) \delta[\mathrm{ppm}]=$ $7.33(\mathrm{~s}, 7 \mathrm{H}), 7.22-7.11(\mathrm{~m}, 4 \mathrm{H}), 6.78(\mathrm{~d}, J=6.3 \mathrm{~Hz}, 4 \mathrm{H}), 4.78(\mathrm{~d}, J=13.8 \mathrm{~Hz}, 1 \mathrm{H}), 4.57(\mathrm{~d}, J=$ $13.8 \mathrm{~Hz}, 1 \mathrm{H}), 3.64(\mathrm{~s}, 3 \mathrm{H}), 1.92(\mathrm{~s}, 3 \mathrm{H}) .{ }^{13} \mathbf{C}$ NMR $\left(101 \mathrm{MHz}, \mathrm{CDCl}_{3}\right) \delta[\mathrm{ppm}]=171.1,159.7$, $139.4,137.8,136.5,135.9,130.3,129.7,128.8,128.6,128.2,127.6,126.8,126.1,121.1,114.8$ $112.5,55.1,50.4,22.0$.

IR (KBr) v (cm-1) 3061, 3029, 2935, 1656, 1492, 1387, 1270, 974, 763, 695.

HRMS (ESI) m/z: $[\mathrm{M}+\mathrm{H}]^{+}$Calcd for $\mathrm{C}_{24} \mathrm{H}_{24} \mathrm{NO}_{2}$ 358.1802; found 358.1816.<smiles>CC(=O)N(Cc1ccccc1)/C(=C\c1ccc2c(c1)OCO2)c1ccccc1</smiles>

(Z)-N-(2-(benzo[d][1,3]dioxol-5-yl)-1-phenylvinyl)- $N$-benzylacetamide (4j)

Following the general procedure (eluent: petroleum ether/ethyl acetate $=10 / 1$ ), the desired product 4j was obtained as a pale yellow oil $(100.1 \mathrm{mg}, 99 \%$ yield $) ;{ }^{1} \mathbf{H}$ NMR $\left(400 \mathrm{MHz}, \mathrm{CDCl}_{3}\right) \delta[\mathrm{ppm}]$ $=7.34-7.27(\mathrm{~m}, 7 \mathrm{H}), 7.22-7.17(\mathrm{~m}, 3 \mathrm{H}), 6.73-6.66(\mathrm{~m}, 3 \mathrm{H}), 6.59(\mathrm{~s}, 1 \mathrm{H}), 5.91(\mathrm{~d}, J=1.2 \mathrm{~Hz}$, 2H), $4.85(\mathrm{~d}, J=13.7 \mathrm{~Hz}, 1 \mathrm{H}), 4.49(\mathrm{~d}, J=13.7 \mathrm{~Hz}, 1 \mathrm{H}), 1.92(\mathrm{~s}, 3 \mathrm{H}) .{ }^{13} \mathbf{C}$ NMR $(101 \mathrm{MHz}$, $\left.\mathrm{CDCl}_{3}\right) \delta[\mathrm{ppm}]=171.1,148.0,147.7,138.0,137.6,136.6,130.4,128.9,128.8,128.4,128.3$, $127.7,126.8,126.0,123.5,108.4,107.7,101.2,50.2,22.0$.

IR (KBr) v (cm-1) 3061, 3029, 2930, 1654, 1489, 1386, 1259, 975, 760, 697.

HRMS (ESI) m/z: $[\mathrm{M}+\mathrm{H}]^{+}$Calcd for $\mathrm{C}_{24} \mathrm{H}_{22} \mathrm{NO}_{3}$ 372.1594; found 372.1610. 
<smiles>CC(=O)N(Cc1ccccc1)/C(=C\c1ccc(F)cc1)c1ccccc1</smiles>

(Z)- $N$-benzyl- $N$-(2-(4-fluorophenyl)-1-phenylvinyl)acetamide (4k)

Following the general procedure (eluent: petroleum ether/ethyl acetate $=15 / 1$ ), the desired product 4k was obtained as a pale yellow oil (76.0 mg ,82\% yield); ${ }^{1} \mathbf{H}$ NMR (400 MHz, $\left.\mathrm{CDCl}_{3}\right) \delta[\mathrm{ppm}]$ $=7.37-7.29(\mathrm{~m}, 7 \mathrm{H}), 7.21-7.16(\mathrm{~m}, 3 \mathrm{H}), 7.09(\mathrm{dd}, J=8.7,5.6 \mathrm{~Hz}, 2 \mathrm{H}), 6.88(\mathrm{t}, J=8.7 \mathrm{~Hz}, 2 \mathrm{H})$, $6.78(\mathrm{~s}, 1 \mathrm{H}), 4.91(\mathrm{~d}, J=13.7 \mathrm{~Hz}, 1 \mathrm{H}), 4.42$ (d, $J=13.7 \mathrm{~Hz}, 1 \mathrm{H}), 1.91(\mathrm{~s}, 3 \mathrm{H}) .{ }^{13} \mathbf{C}$ NMR (101 $\left.\mathrm{MHz}, \mathrm{CDCl}_{3}\right) \delta[\mathrm{ppm}]=170.7,162.1\left(\mathrm{~d},{ }^{1} J_{C-F}=249.7 \mathrm{~Hz}\right), 138.7,137.5,136.3,130.6\left(\mathrm{~d},{ }^{4} J_{C-F}=\right.$ $3.6 \mathrm{~Hz}), 130.2,129.9\left(\mathrm{~d},{ }^{3} J_{C-F}=8.0 \mathrm{~Hz}\right), 128.7,128.6,128.1,127.6,125.9,125.8,115.5\left(\mathrm{~d},{ }^{2} J_{C-F}=\right.$ $21.5 \mathrm{~Hz}), 50.1,21.7$.

IR (KBr) v (cm-1) 3062, 3031, 2930, 1658, 1507, 1387, 1236, 975, 758, 698.

HRMS (ESI) m/z: $[\mathrm{M}+\mathrm{H}]^{+}$Calcd for $\mathrm{C}_{23} \mathrm{H}_{21} \mathrm{FNO} 346.1602$; found 346.1613 .<smiles>CC(=O)N(Cc1ccccc1)/C(=C\c1ccc(Cl)cc1)c1ccccc1</smiles>

(Z)-N-benzyl-N-(2-(4-chlorophenyl)-1-phenylvinyl)acetamide (4I)

Following the general procedure (eluent: petroleum ether/ethyl acetate $=20 / 1$ ), the desired product $4 \mathbf{l}$ was obtained as a pale yellow oil ( $86.0 \mathrm{mg}, 88 \%$ yield); ${ }^{1} \mathbf{H} \mathbf{~ N M R ~}\left(400 \mathrm{MHz}, \mathrm{CDCl}_{3}\right) \delta[\mathrm{ppm}]=$ $7.38-7.29(\mathrm{~m}, 7 \mathrm{H}), 7.23-7.17(\mathrm{~m}, 3 \mathrm{H}), 7.15(\mathrm{~d}, J=8.6 \mathrm{~Hz}, 2 \mathrm{H}), 7.01(\mathrm{~d}, J=8.6 \mathrm{~Hz}, 2 \mathrm{H}), 6.76$ (s, 1H), $4.94(\mathrm{~d}, J=13.7 \mathrm{~Hz}, 1 \mathrm{H}), 4.40(\mathrm{~d}, J=13.6 \mathrm{~Hz}, 1 \mathrm{H}), 1.91(\mathrm{~s}, 3 \mathrm{H}) .{ }^{13} \mathbf{C}$ NMR (101 MHz, $\left.\mathrm{CDCl}_{3}\right) \delta[\mathrm{ppm}]=171.0,139.7,137.5,136.3,134.0,133.1,130.3,129.4,129.3,129.0,128.9$, $128.4,127.9,126.2,125.9,50.4,21.9$.

IR (KBr) v (cm-1) 3061, 3029, 2930, 1659, 1493, 1387, 1242, 975, 761, 698.

HRMS (ESI) m/z: $[\mathrm{M}+\mathrm{H}]^{+}$Calcd for $\mathrm{C}_{23} \mathrm{H}_{21} \mathrm{ClNO} 362.1306$; found 362.1320 . 
<smiles>CC(=O)N(Cc1ccccc1)/C(=C\c1ccc(C(F)(F)F)cc1)c1ccccc1</smiles>

(Z)- $N$-benzyl- $N$-(1-phenyl-2-(4-(trifluoromethyl)phenyl)vinyl)acetamide (4m)

Following the general procedure (eluent: petroleum ether/ethyl acetate $=15 / 1$ ), the desired product 4m was obtained as a pale yellow oil $\left(73.9 \mathrm{mg}, 70 \%\right.$ yield); ${ }^{1} \mathbf{H} \mathbf{~ N M R}\left(400 \mathrm{MHz}, \mathrm{CDCl}_{3}\right) \delta[\mathrm{ppm}]$ $=7.40(\mathrm{~d}, J=4.9 \mathrm{~Hz}, 7 \mathrm{H}), 7.34-7.29(\mathrm{~m}, 2 \mathrm{H}), 7.21(\mathrm{dt}, J=4.8,1.7 \mathrm{~Hz}, 3 \mathrm{H}), 7.15(\mathrm{~d}, J=8.2 \mathrm{~Hz}$, 2H), $6.83(\mathrm{~s}, 1 \mathrm{H}), 4.99(\mathrm{~d}, J=13.7 \mathrm{~Hz}, 1 \mathrm{H}), 4.35(\mathrm{~d}, J=13.7 \mathrm{~Hz}, 1 \mathrm{H}), 1.90(\mathrm{~s}, 3 \mathrm{H}) .{ }^{13} \mathbf{C}$ NMR $\left(101 \mathrm{MHz}, \mathrm{CDCl}_{3}\right) \delta[\mathrm{ppm}]=170.6,141.3,138.0,137.2,136.2,130.3,129.7\left(\mathrm{~d},{ }^{2} J_{C-F}=32.6 \mathrm{~Hz}\right)$, $129.2,129.0,128.3,128.2,127.9,126.3,125.6,125.5\left(\mathrm{q},{ }^{3} J_{C-F}=3.8 \mathrm{~Hz}\right), 123.8\left(\mathrm{~d},{ }^{1} J_{C-F}=272.0\right.$ $\mathrm{Hz}), 50.3,21.8$.

IR (KBr) v (cm-1) 3063, 3032, 2934, 1664, 1493, 1389, 1241, 976, 757, 698.

HRMS (ESI) m/z: $[\mathrm{M}+\mathrm{H}]^{+}$Calcd for $\mathrm{C}_{24} \mathrm{H}_{21} \mathrm{~F}_{3} \mathrm{NO}$ 396.1570; found 396.1583 .<smiles>CC(=O)N(Cc1ccccc1)/C(=C\c1ccc(-c2ccccc2)cc1)c1ccccc1</smiles>

(Z)- $N$-(2-([1,1'-biphenyl]-4-yl)-1-phenylvinyl)- $N$-benzylacetamide (4n)

Following the general procedure (eluent: petroleum ether/ethyl acetate $=15 / 1$ ), the desired product 4n was obtained as a pale yellow oil $\left(107.1 \mathrm{mg}, 98 \%\right.$ yield); ${ }^{1} \mathbf{H} \mathbf{~ N M R}\left(400 \mathrm{MHz}, \mathrm{CDCl}_{3}\right) \delta[\mathrm{ppm}]$ $=7.62-7.55(\mathrm{~m}, 2 \mathrm{H}), 7.51-7.41(\mathrm{~m}, 4 \mathrm{H}), 7.40-7.31(\mathrm{~m}, 8 \mathrm{H}), 7.27-7.17(\mathrm{~m}, 5 \mathrm{H}), 6.86(\mathrm{~s}, 1 \mathrm{H})$, $4.89(\mathrm{~d}, J=13.7 \mathrm{~Hz}, 1 \mathrm{H}), 4.56(\mathrm{~d}, J=13.7 \mathrm{~Hz}, 1 \mathrm{H}), 1.96(\mathrm{~s}, 3 \mathrm{H}) .{ }^{13} \mathbf{C}$ NMR $\left(101 \mathrm{MHz}, \mathrm{CDCl}_{3}\right) \delta$ $[\mathrm{ppm}]=171.1,140.8,140.1,139.0,137.8,136.4,133.5,130.3,128.8,128.8,128.7,128.6,128.2$, 127.7, 127.5, 127.3, 126.8, 126.6, 126.1, 50.3, 22.0.

IR (KBr) v (cm-1) 3059, 3029, 2932, 1655, 1491, 1387, 1242, 975, 763, 696. 
HRMS (ESI) m/z: [M+H] $]^{+}$Calcd for $\mathrm{C}_{29} \mathrm{H}_{25} \mathrm{NO}$ 404.2009; found 404.2009.<smiles>CC(=O)N(Cc1ccccc1)/C(=C\c1cccc2ccccc12)c1ccccc1</smiles>

(Z)- $N$-benzyl- $N$-(2-(naphthalen-1-yl)-1-phenylvinyl)acetamide (4o)

Following the general procedure (eluent: petroleum ether/ethyl acetate $=20 / 1$ ), the desired product 4o was obtained as a pale yellow oil (91.7 $\mathrm{mg}, 90 \%$ yield); ${ }^{1} \mathbf{H}$ NMR (400 MHz, $\left.\mathrm{CDCl}_{3}\right) \delta[\mathrm{ppm}]$ $=8.01(\mathrm{dd}, J=6.2,3.5 \mathrm{~Hz}, 1 \mathrm{H}), 7.86(\mathrm{dd}, J=6.2,3.3 \mathrm{~Hz}, 1 \mathrm{H}), 7.77(\mathrm{~d}, J=8.2 \mathrm{~Hz}, 1 \mathrm{H}), 7.51(\mathrm{dt}$, $J=6.4,3.4 \mathrm{~Hz}, 2 \mathrm{H}), 7.39-7.33(\mathrm{~m}, 6 \mathrm{H}), 7.31-7.27(\mathrm{~m}, 2 \mathrm{H}), 7.27-7.20(\mathrm{~m}, 4 \mathrm{H}), 6.94(\mathrm{~d}, J=$ $7.2 \mathrm{~Hz}, 1 \mathrm{H}), 4.62(\mathrm{~d}, J=13.7 \mathrm{~Hz}, 1 \mathrm{H}), 4.51(\mathrm{~d}, J=13.8 \mathrm{~Hz}, 1 \mathrm{H}), 1.84(\mathrm{~s}, 3 \mathrm{H}) .{ }^{13} \mathbf{C}$ NMR $(101$ $\left.\mathrm{MHz}, \mathrm{CDCl}_{3}\right) \delta[\mathrm{ppm}]=171.4,141.5,138.5,137.0,133.8,131.6,131.5,130.3,128.9,128.8$, $128.8,128.4,127.7,126.5,126.5,126.0,125.8,125.2,124.0,123.5,50.6,22.7$.

IR (KBr) v (cm-1) 3059, 3030, 2929, 1658, 1493, 1385, 1233, 976, 762, 698.

HRMS (ESI) m/z: $[\mathrm{M}+\mathrm{H}]^{+}$Calcd for $\mathrm{C}_{27} \mathrm{H}_{23} \mathrm{NO} 378.1852$; found 378.1870 .<smiles>CC(=O)N(Cc1ccccc1)C(=Cc1ccsc1)c1ccccc1</smiles>

(Z)-N-benzyl- $N$-(1-phenyl-2-(thiophen-3-yl)vinyl)acetamide (4p)

Following the general procedure (eluent: petroleum ether/ethyl acetate $=15 / 1$ ), the desired product $4 \mathbf{p}$ was obtained as a pale yellow oil (43.2 $\mathrm{mg}, 48 \%$ yield); ${ }^{1} \mathbf{H} \mathbf{~ N M R}\left(400 \mathrm{MHz}, \mathrm{CDCl}_{3}\right) \delta[\mathrm{ppm}]$ $=7.37-7.26(\mathrm{~m}, 7 \mathrm{H}), 7.19-7.12(\mathrm{~m}, 5 \mathrm{H}), 6.93(\mathrm{~s}, 1 \mathrm{H}), 6.87(\mathrm{dd}, J=4.9,1.6 \mathrm{~Hz}, 1 \mathrm{H}), 4.88(\mathrm{~d}, J$ $=13.7 \mathrm{~Hz}, 1 \mathrm{H}), 4.48(\mathrm{~d}, J=13.7 \mathrm{~Hz}, 1 \mathrm{H}), 1.93(\mathrm{~s}, 3 \mathrm{H}) .{ }^{13} \mathbf{C} \mathbf{N M R}\left(101 \mathrm{MHz}, \mathrm{CDCl}_{3}\right) \delta[\mathrm{ppm}]=$ 171.1, 137.6, 137.2, 136.4, 135.9, 130.2, 128.8, 128.4, 128.1, 127.6, 127.0, 125.9, 125.8, 125.5, 121.5, 50.2, 21.7 .

IR (KBr) v (cm-1) 3086, 3061, 3030, 2931, 1652, 1492, 1389, 1237, 1030, 976, 697. 
HRMS (ESI) m/z: [M+H] $]^{+}$Calcd for $\mathrm{C}_{21} \mathrm{H}_{20} \mathrm{NOS} 334.1260$; found 334.1268.<smiles>CC(=O)N(Cc1ccccc1)C(Cc1ccccc1)c1ccccc1</smiles>

$N$-benzyl- $N$-(1,2-diphenylethyl)acetamide (5)

Following the general procedure (eluent: petroleum ether/ethyl acetate $=15 / 1$ ), the desired product 5 was obtained as a pale yellow oil $\left(58.0 \mathrm{mg}, 88 \%\right.$ yield); ${ }^{1} \mathbf{H}$ NMR $\left(400 \mathrm{MHz}, \mathrm{CDCl}_{3}\right)$ for two conformers: $\delta[\mathrm{ppm}]=7.54-6.84(\mathrm{~m}, 15 \mathrm{H}), 6.20$ and $5.28(2 \times \mathrm{t}, 1 \mathrm{H}), 4.68$ and $4.49(2 \times \mathrm{d}, 1 \mathrm{H})$, $4.30(\mathrm{~d}, J=17.0 \mathrm{~Hz}, 1 \mathrm{H}), 3.38$ and $3.25(2 \times \mathrm{q}, 2 \mathrm{H}), 1.95(\mathrm{~d}, J=13.7 \mathrm{~Hz}, 3 \mathrm{H}) .{ }^{13} \mathbf{C} \mathbf{N M R}(101$ $\left.\mathrm{MHz}, \mathrm{CDCl}_{3}\right) \delta[\mathrm{ppm}]=171.7,171.2,139.1,138.8,138.1,137.8,137.4,129.1,128.9,128.5$, $128.3,128.3,128.2,127.9,127.7,127.6,126.9,126.7,126.5,126.2,126.1,63.3,58.2,48.8,45.8$, $37.7,37.2,22.6,21.9$.

IR (KBr) v (cm-1) 3063, 3028, 2921, 1625, 1493, 1361, 1259, 1027, 959, 696.

HRMS (ESI) m/z: $[\mathrm{M}+\mathrm{H}]^{+}$Calcd for $\mathrm{C}_{23} \mathrm{H}_{24} \mathrm{NO} 330.1852$; found 330.1859 .<smiles>CC(=O)N(Cc1ccccc1)C(=C(c1ccccc1)c1ccccc1)c1ccccc1</smiles>

$N$-benzyl- $N$-(1,2,2-triphenylvinyl)acetamide (6)

Following the general procedure (eluent: petroleum ether/ethyl acetate $=15 / 1$ ), the desired product 6 was obtained as a pale yellow oil (49.2 mg, 61\% yield); ${ }^{\mathbf{1}} \mathbf{H} \mathbf{~ N M R}\left(400 \mathrm{MHz}, \mathrm{CDCl}_{3}\right) \delta[\mathrm{ppm}]=$ $7.33-7.29(\mathrm{~m}, 5 \mathrm{H}), 7.23(\mathrm{dd}, J=5.2,2.0 \mathrm{~Hz}, 1 \mathrm{H}), 7.14-6.99(\mathrm{~m}, 6 \mathrm{H}), 6.97-6.84(\mathrm{~m}, 6 \mathrm{H}), 4.70$ $(\mathrm{d}, J=13.9 \mathrm{~Hz}, 1 \mathrm{H}), 4.25(\mathrm{~d}, J=13.9 \mathrm{~Hz}, 1 \mathrm{H}), 1.98(\mathrm{~s}, 3 \mathrm{H}) .{ }^{13} \mathbf{C} \mathbf{N M R}\left(101 \mathrm{MHz}, \mathrm{CDCl}_{3}\right) \delta[\mathrm{ppm}]$ $=171.4,142.0,141.2,139.3,138.0,137.9,137.6,131.3,130.4,130.4,129.2,128.5,128.4,128.0$, $128.0,127.9,127.7,127.6,127.1,50.5,23.1$.

IR (KBr) v (cm-1) 3055, 3027, 2956, 2924, 1662, 1491, 1377, 1232, 1027, 985, 697.

HRMS (ESI) m/z: [M+H] $]^{+}$Calcd for $\mathrm{C}_{29} \mathrm{H}_{26} \mathrm{NO} 404.2009$; found 404.2019. 


\section{References}

[1] Guan, Z.-H.; Zhang, Z.-Y.; Ren, Z.-H.; Wang, Y.-Y.; Zhang, X. J. Org. Chem. 2011, 76, 339-341.

[2] Yu, W.; Chen, J.; Gao, K.; Liu, Z.; Zhang, Y. Org. Lett. 2014, 16, 4870-4873.

[3] Pankajakshan, S.; Xu, Y.-H.; Cheng, J. K.; Low, M. T.; Loh, T.-P. Angew. Chem., Int. Ed. 2012, 51, 57015705.

[4] Chang, X.-H.; Wang, Z.-L.; Zhao, M.; Yang, C.; Li, J.-J.; Ma, W.-W.; Xu, Y.-H. Org. Lett. 2020, 22, 13261330.

[5] Manoso, A. S.; Ahn, C.; Soheili, A.; Handy, C. J.; Correia, R.; Seganish, W. M.; DeShong, P. J. Org. Chem. 2004, 69, 8305-8314.

[6] Yu, Jingxun.; Liu, Jun.; Shi, Guangfa.; Shao, Changdong.; Zhang, Yanghui. Angew. Chem., Int. Ed. 2015, 54, $4079-4082$.

[7] Qiao, B.; Cao, H.-Q.; Huang, Y.-J.; Zhang, Y.; Nie, J.; Zhang, F.-G.; Ma, J.-A. Chin. J. Chem. 2018, 36, 809814.

[8] Brettle, R.; Shibib, S. M.; Wheeler, K. J. J. Chem. Soc., Perkin Trans. 1. 1985, 831-836.

[9] Tatsushi Imahori.; Chieko Hori.; Yoshinori Kondo. Adv. Synth. Catal. 2004, 346, 1090-1092. 


\section{5、 ${ }^{1} \mathrm{H}$ NMR, ${ }^{13} \mathrm{C}$ NMR spectra}

$N$-benzyl- $N$-(1-(2,4-dimethoxyphenyl)vinyl)acetamide (1f) ${ }^{1} \mathrm{H} \mathrm{NMR}\left(400 \mathrm{MHz}, \mathrm{CDCl}_{3}\right)$

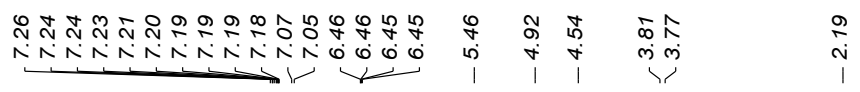<smiles>C=C(c1ccc(OC)cc1OC)N(Cc1ccccc1)C(C)=O</smiles>

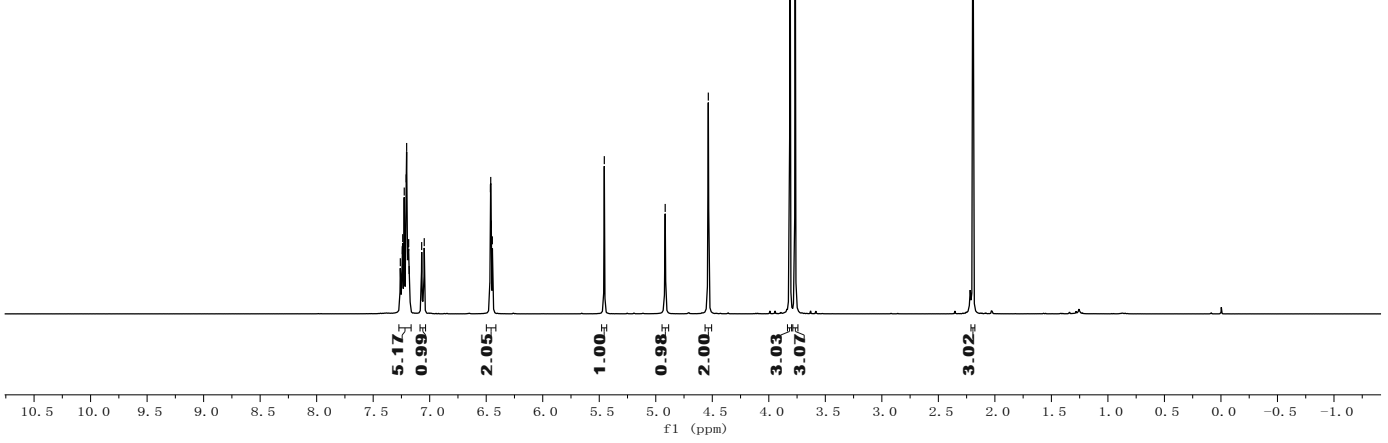

$N$-benzyl- $N$-(1-(2,4-dimethoxyphenyl)vinyl)acetamide (1f)

${ }^{13} \mathrm{C}$ NMR $\left(101 \mathrm{MHz}, \mathrm{CDCl}_{3}\right)$

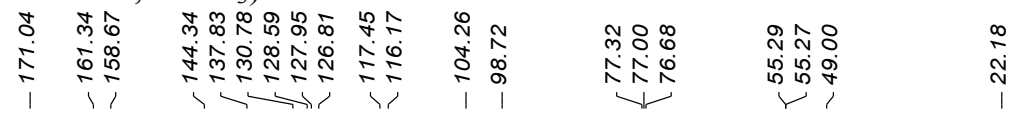

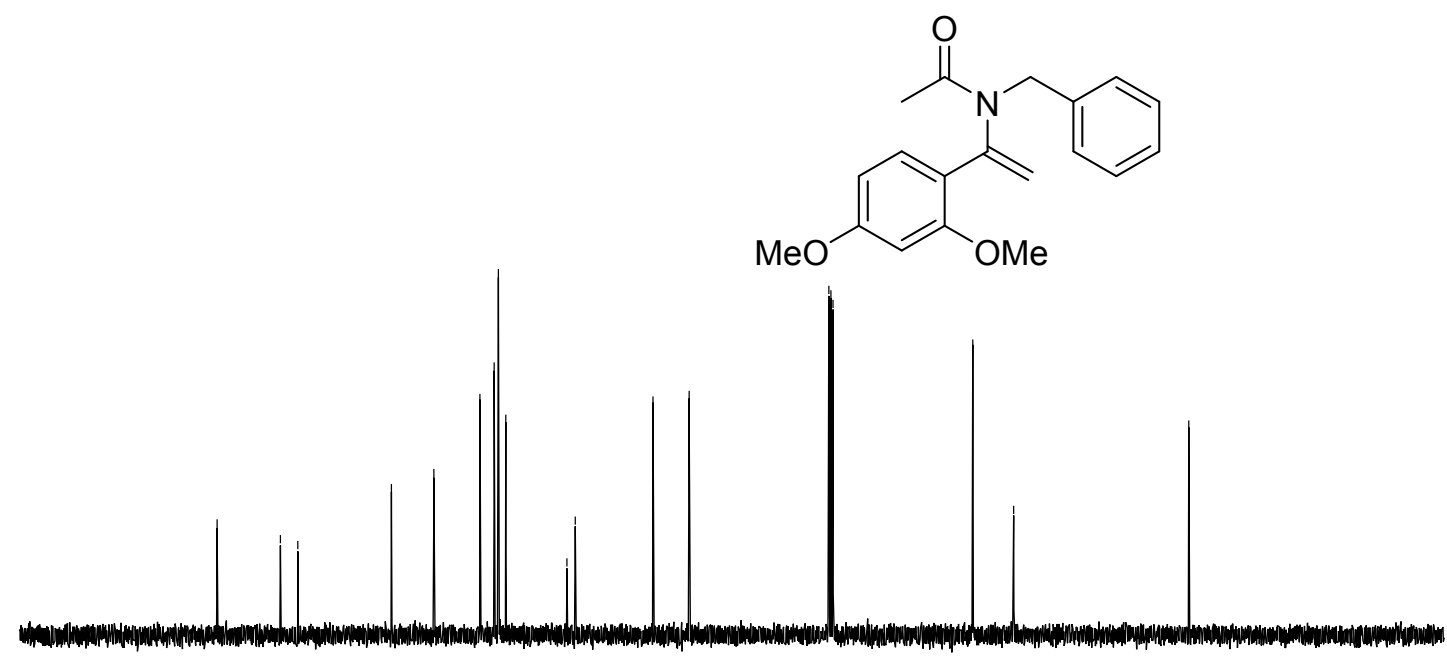

$N$-(1-(benzo[d][1,3]dioxol-5-yl)vinyl)- $N$-benzylacetamide (1h) 
${ }^{1} \mathrm{H}$ NMR (400 MHz, $\mathrm{CDCl}_{3}$ )

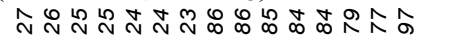

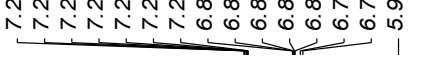

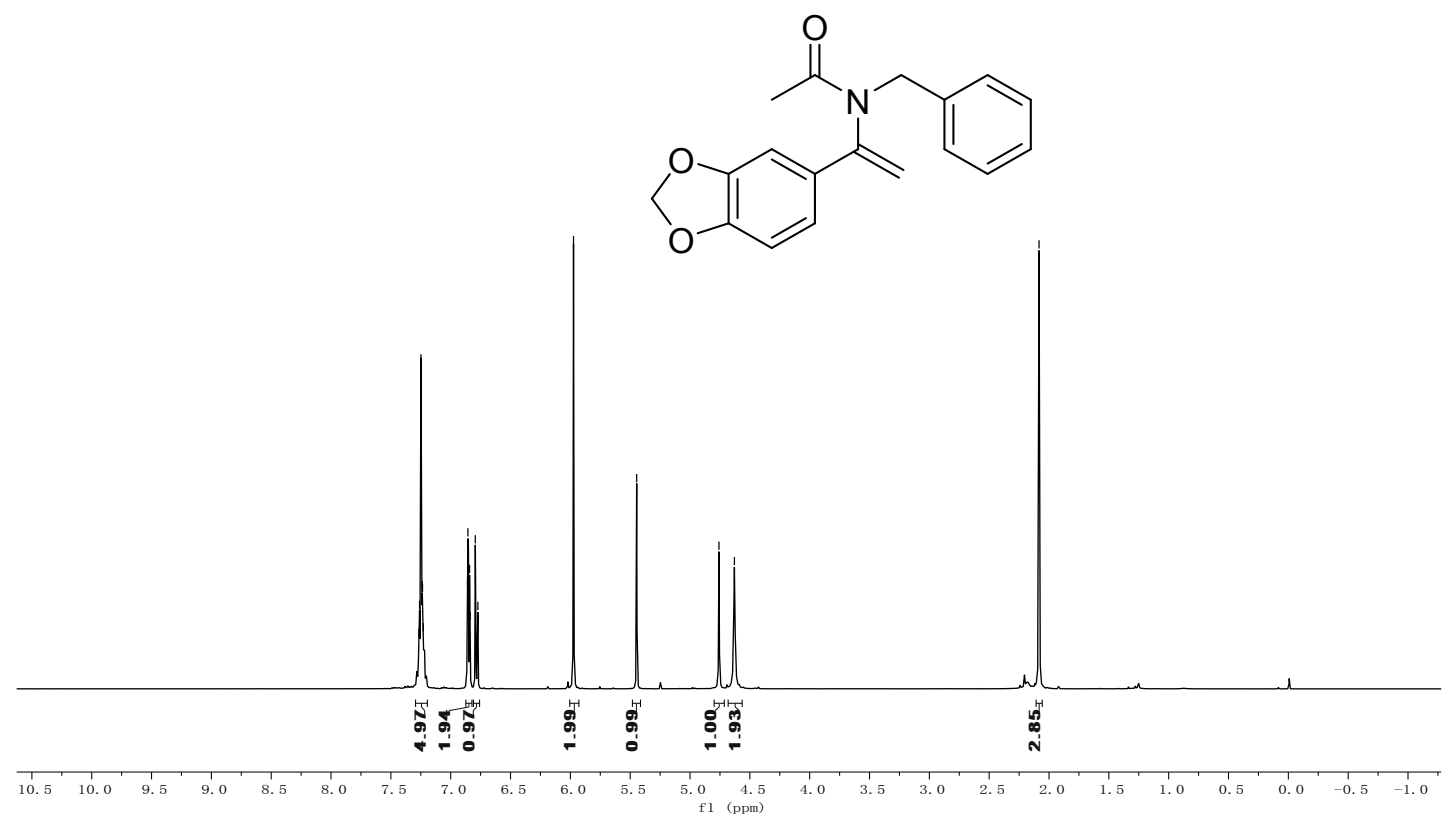

$N$-(1-(benzo[d][1,3]dioxol-5-yl)vinyl)- $N$-benzylacetamide (1h) ${ }^{13} \mathrm{C} \mathrm{NMR}\left(101 \mathrm{MHz}, \mathrm{CDCl}_{3}\right)$

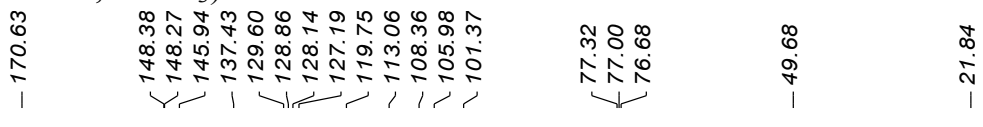

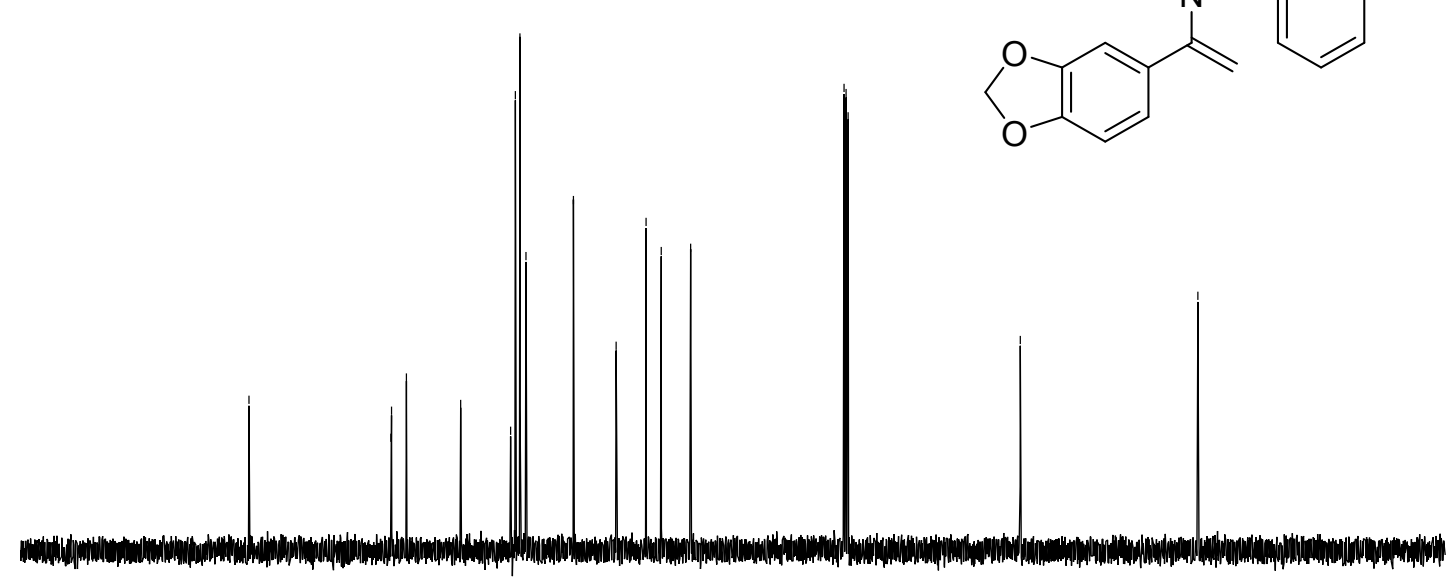<smiles>C=C(c1ccc2c(c1)OCO2)N(Cc1ccccc1)C(C)=O</smiles>

$N$-benzyl- $N$-(1-(4-bromophenyl)vinyl)acetamide (1k)

${ }^{1} \mathrm{H} \mathrm{NMR}\left(400 \mathrm{MHz}, \mathrm{CDCl}_{3}\right)$ 
<smiles>C=C(c1ccc(Br)cc1)N(Cc1ccccc1)C(C)=O</smiles>

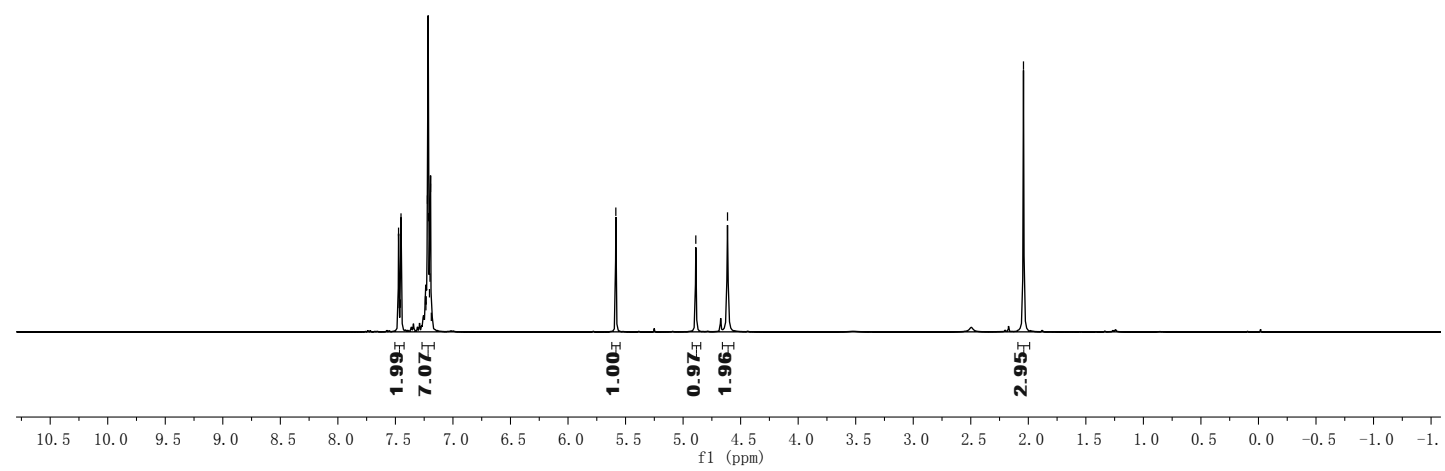

$N$-benzyl- $N$-(1-(4-bromophenyl)vinyl)acetamide (1k)

${ }^{13} \mathrm{C}$ NMR (101 MHz, $\mathrm{CDCl}_{3}$ )

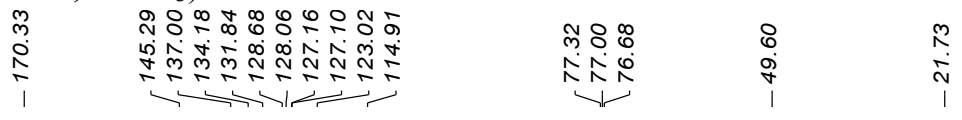

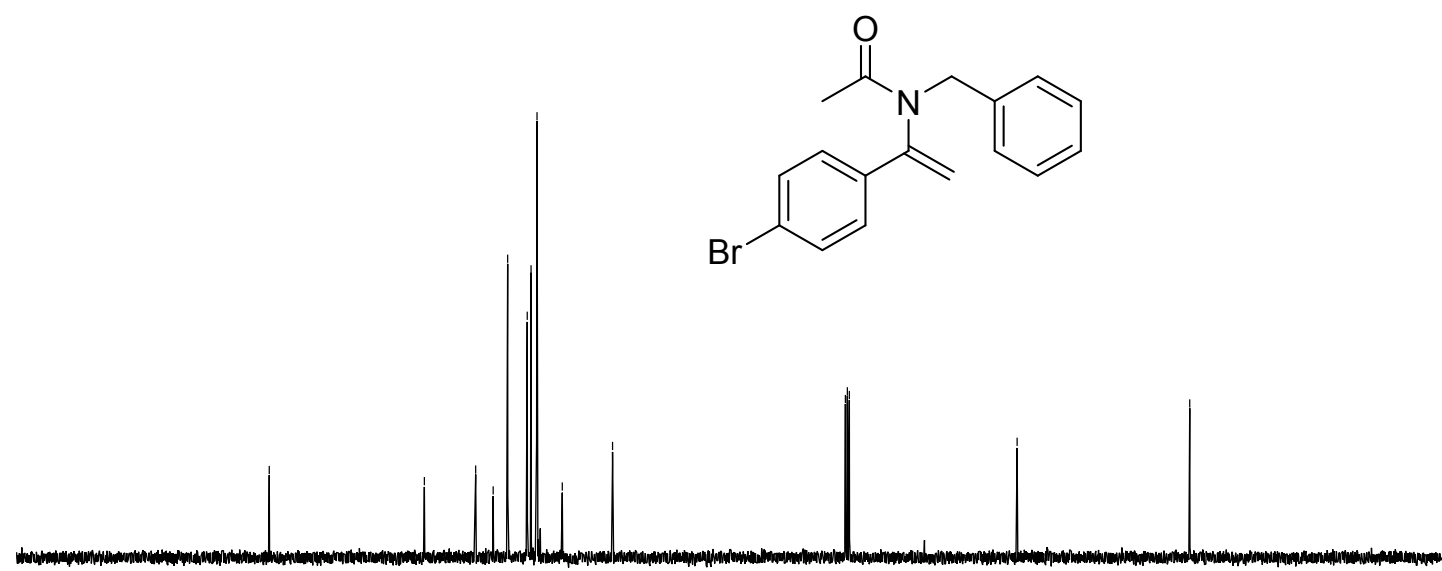

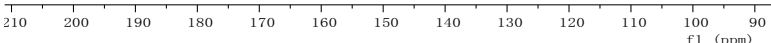

$N$-benzyl- $N$-(1-(3-bromophenyl)vinyl)acetamide (11)

${ }^{1} \mathrm{H}$ NMR (400 MHz, $\mathrm{CDCl}_{3}$ ) 
<smiles>C=C(c1cccc(Br)c1)N(Cc1ccccc1)C(C)=O</smiles>

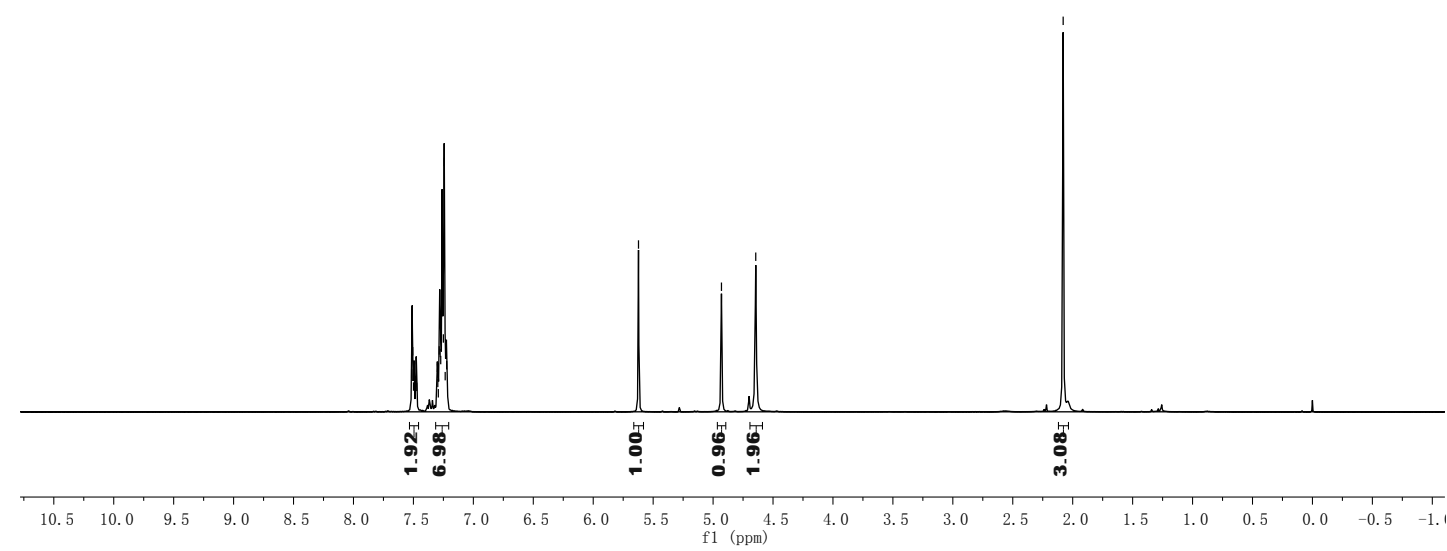

$N$-benzyl- $N$-(1-(3-bromophenyl)vinyl)acetamide (11) ${ }^{13} \mathrm{C}$ NMR (101 MHz, $\left.\mathrm{CDCl}_{3}\right)$

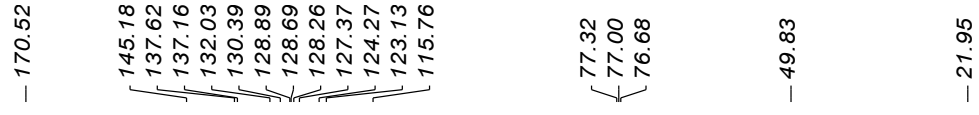

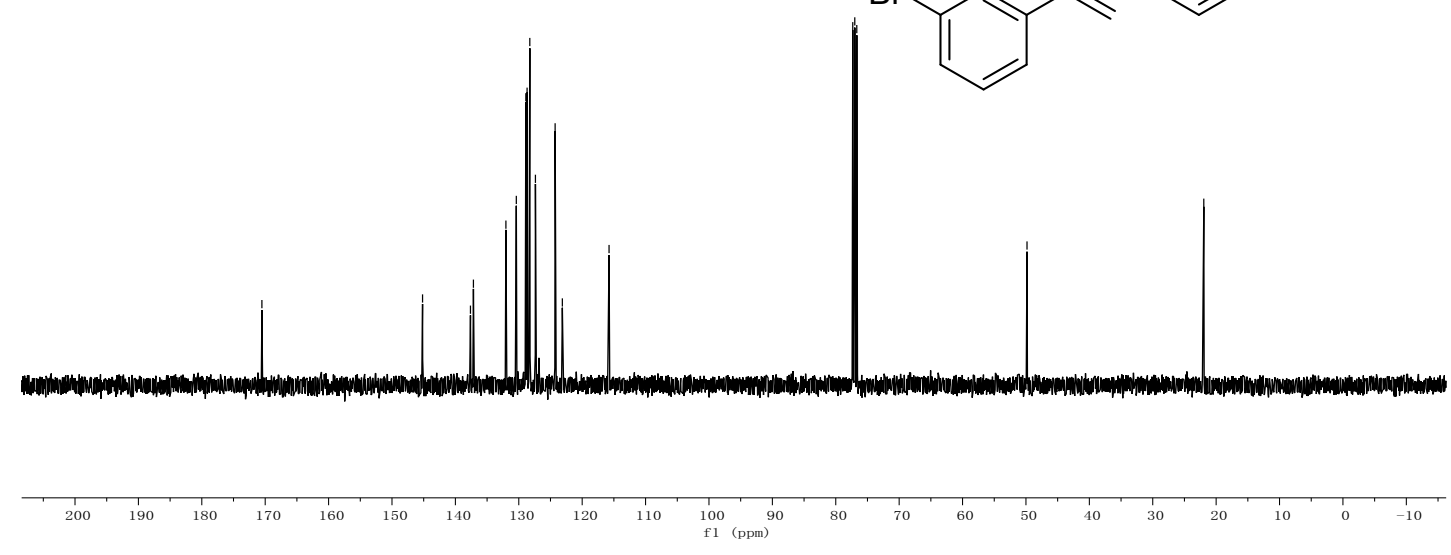

$N$-benzyl- $N$-(1-(2-bromophenyl)vinyl)acetamide (1 $\mathbf{m})$

${ }^{1} \mathrm{H}$ NMR (400 MHz, $\mathrm{CDCl}_{3}$ ) 
<smiles>C=C(c1ccccc1Br)N(Cc1ccccc1)C(C)=O</smiles>

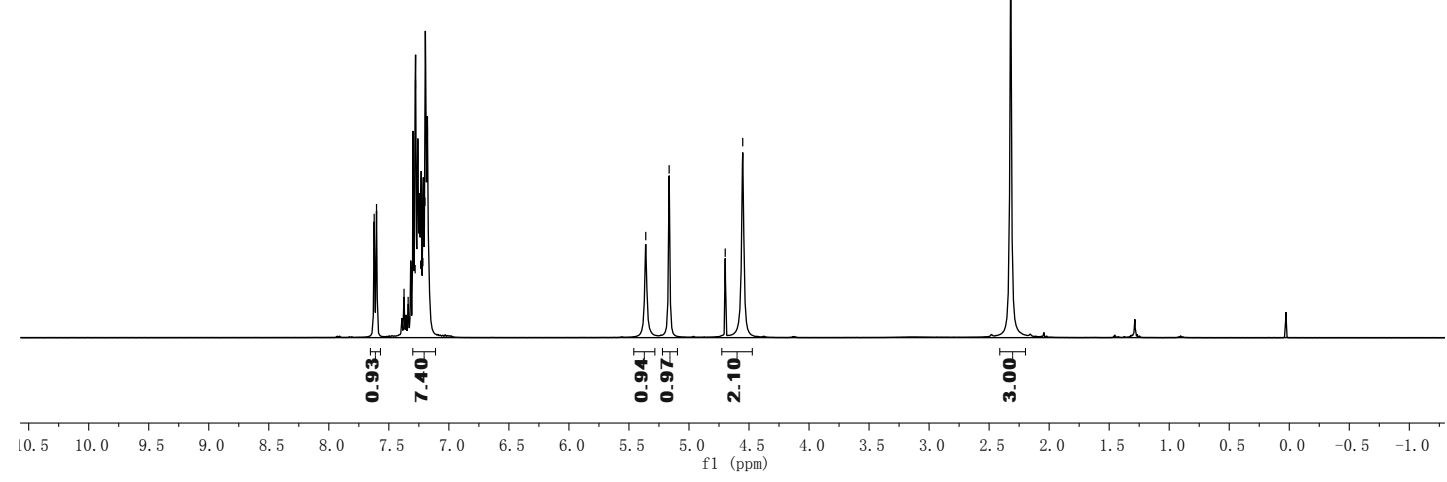

$N$-benzyl- $N$-(1-(2-bromophenyl)vinyl)acetamide (1 m) ${ }^{13} \mathrm{C} \mathrm{NMR}\left(101 \mathrm{MHz}, \mathrm{CDCl}_{3}\right)$

\begin{tabular}{|c|c|c|c|}
\hline $\begin{array}{l}0 \\
0 \\
0 \\
2\end{array}$ & 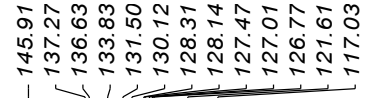 & 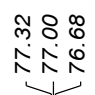 & $\begin{array}{l}\hat{0} \\
\text { के } \\
\infty \\
\text { के } \\
\end{array}$ \\
\hline
\end{tabular}

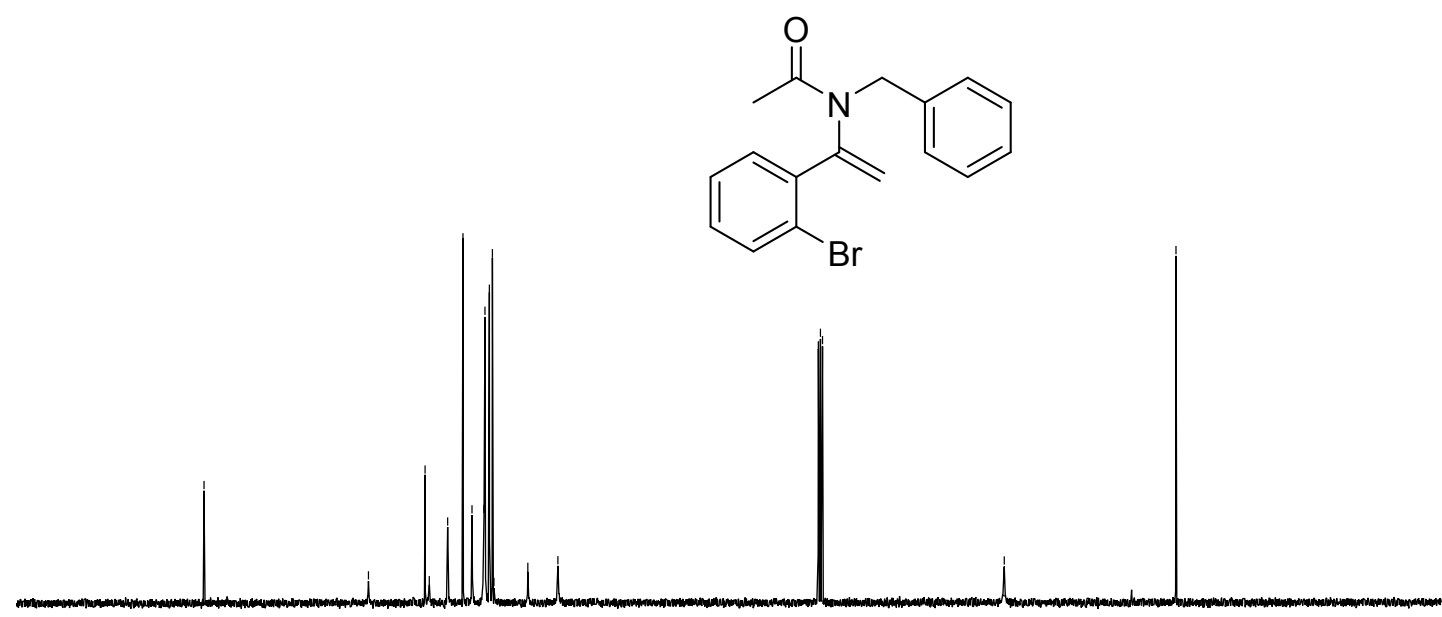

$N$-benzyl- $N$-(1-(4-(trifluoromethyl)phenyl)vinyl)acetamide (1n)

${ }^{1} \mathrm{H} \mathrm{NMR}\left(400 \mathrm{MHz}, \mathrm{CDCl}_{3}\right)$ 


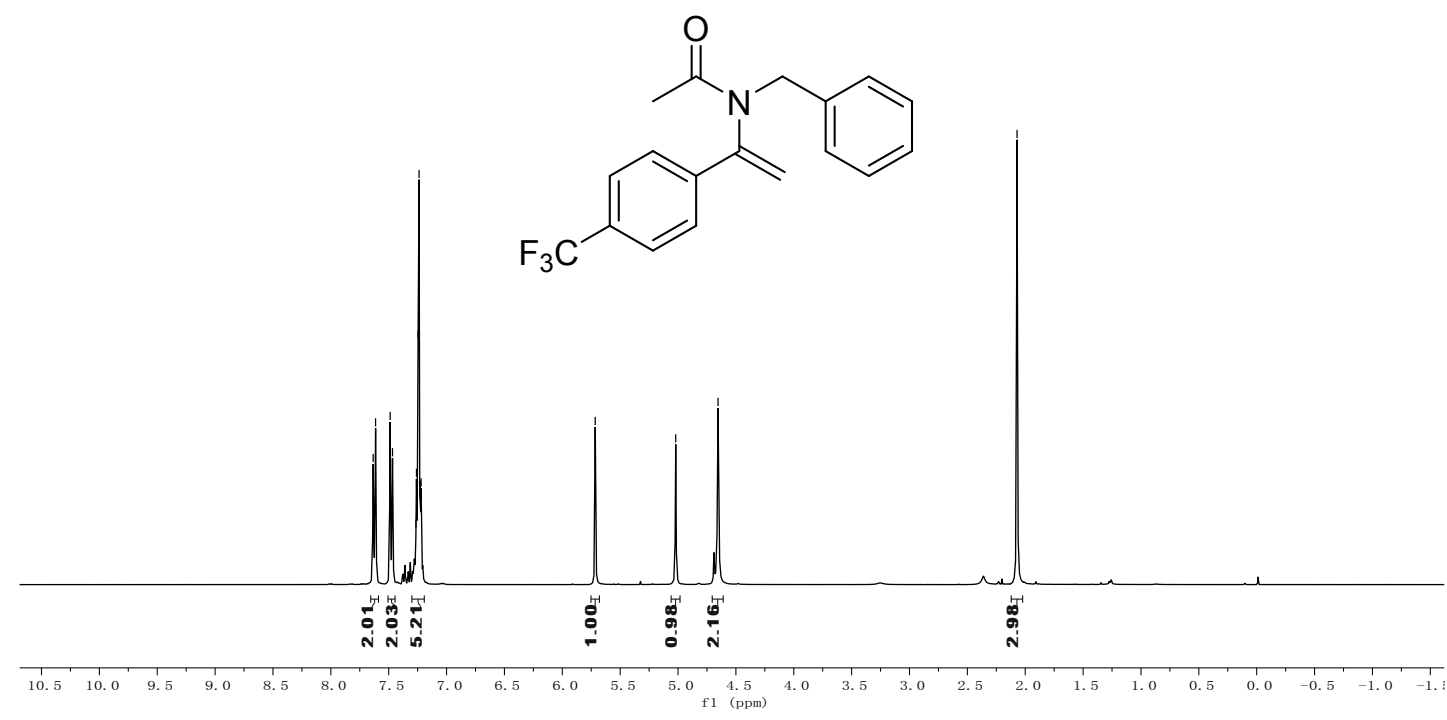

$N$-benzyl- $N$-(1-(4-(trifluoromethyl)phenyl)vinyl)acetamide (1n)

${ }^{13} \mathrm{C}$ NMR (101 MHz, $\left.\mathrm{CDCl}_{3}\right)$

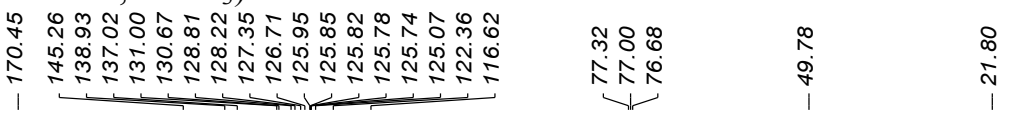
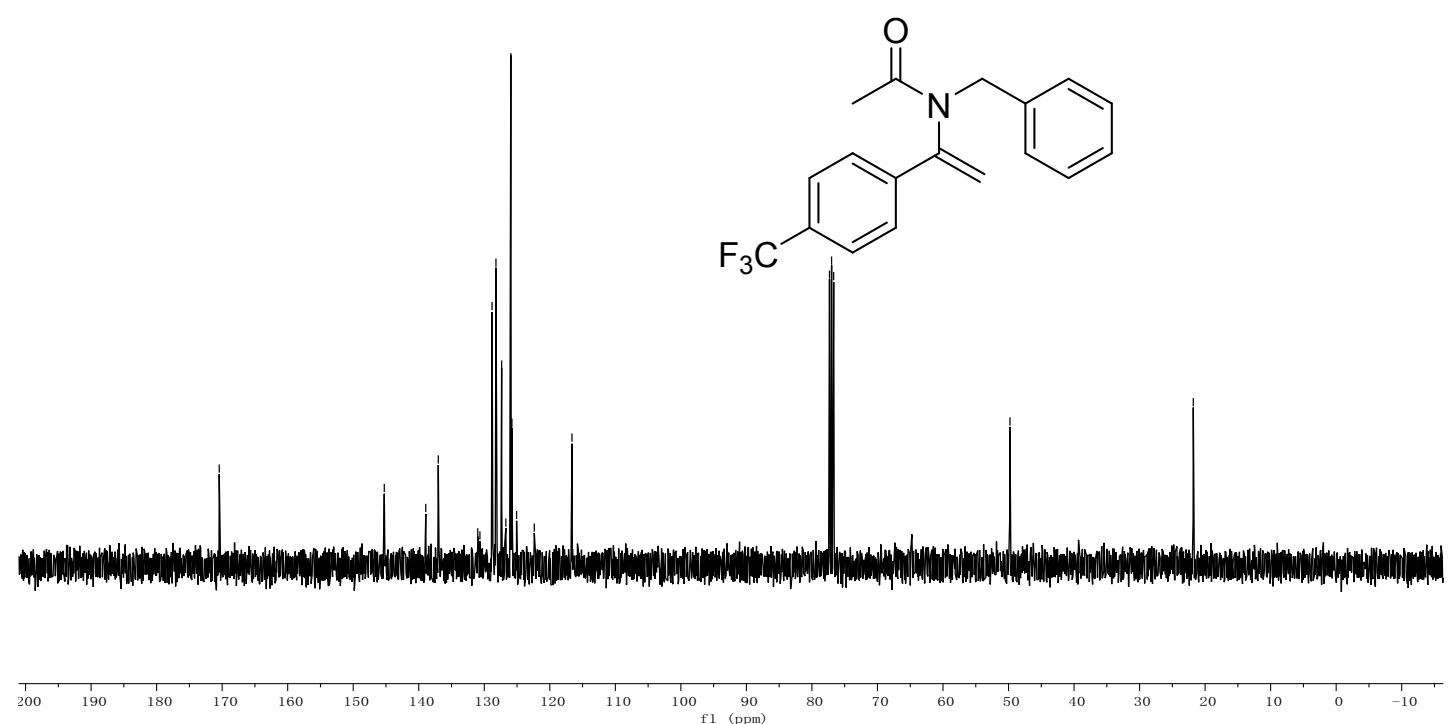

$N$-benzyl- $N$-(1-(4-nitrophenyl)vinyl)acetamide (1p)

${ }^{1} \mathrm{H}$ NMR (400 MHz, $\mathrm{CDCl}_{3}$ ) 


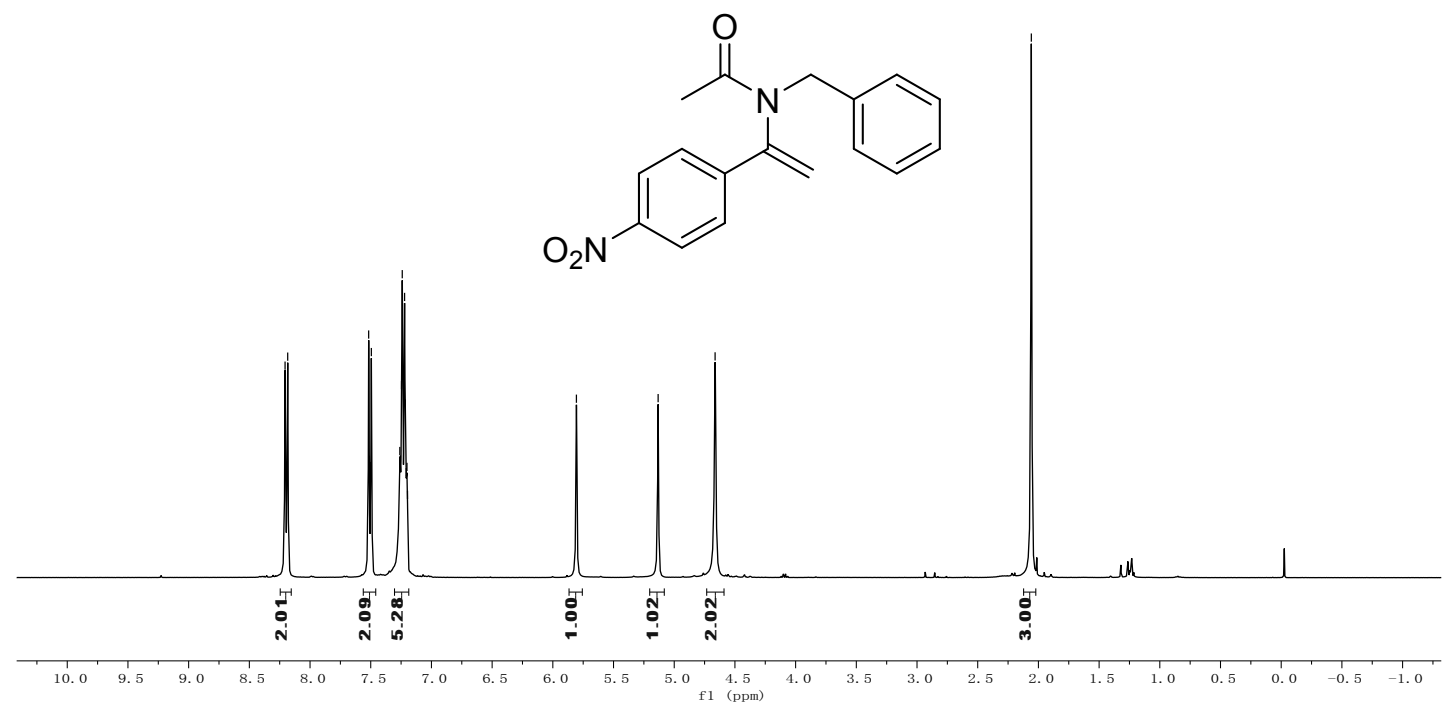

$N$-benzyl- $N$-(1-(4-nitrophenyl)vinyl)acetamide (1p) ${ }^{13} \mathrm{C} \mathrm{NMR}\left(101 \mathrm{MHz}, \mathrm{CDCl}_{3}\right)$

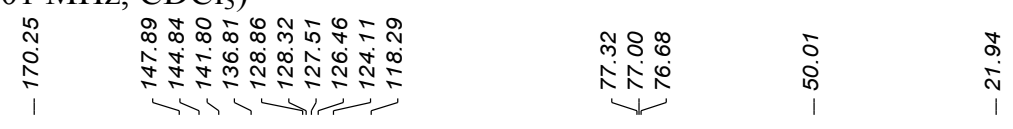
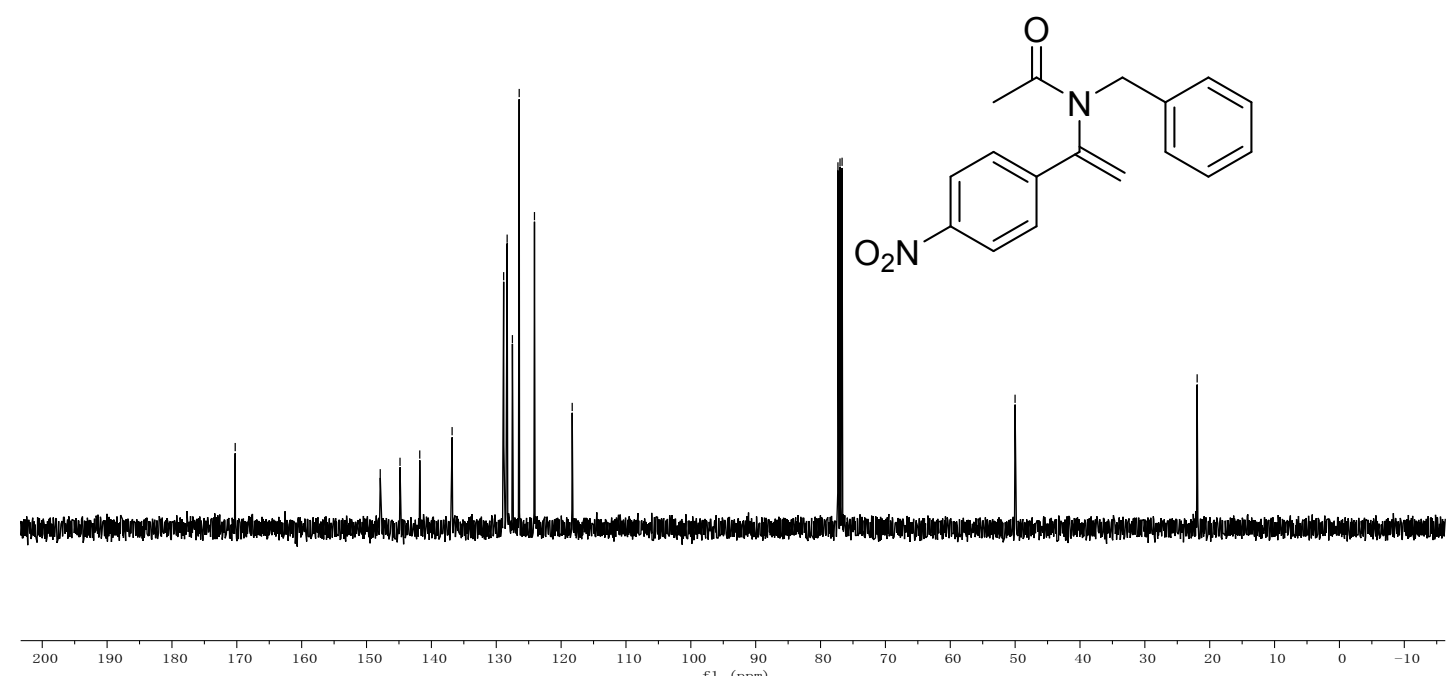

(Z)- $N$-benzyl- $N$-(1,2-diphenylvinyl)acetamide (3a)

${ }^{1} \mathrm{H}$ NMR (400 MHz, $\mathrm{CDCl}_{3}$ ) 


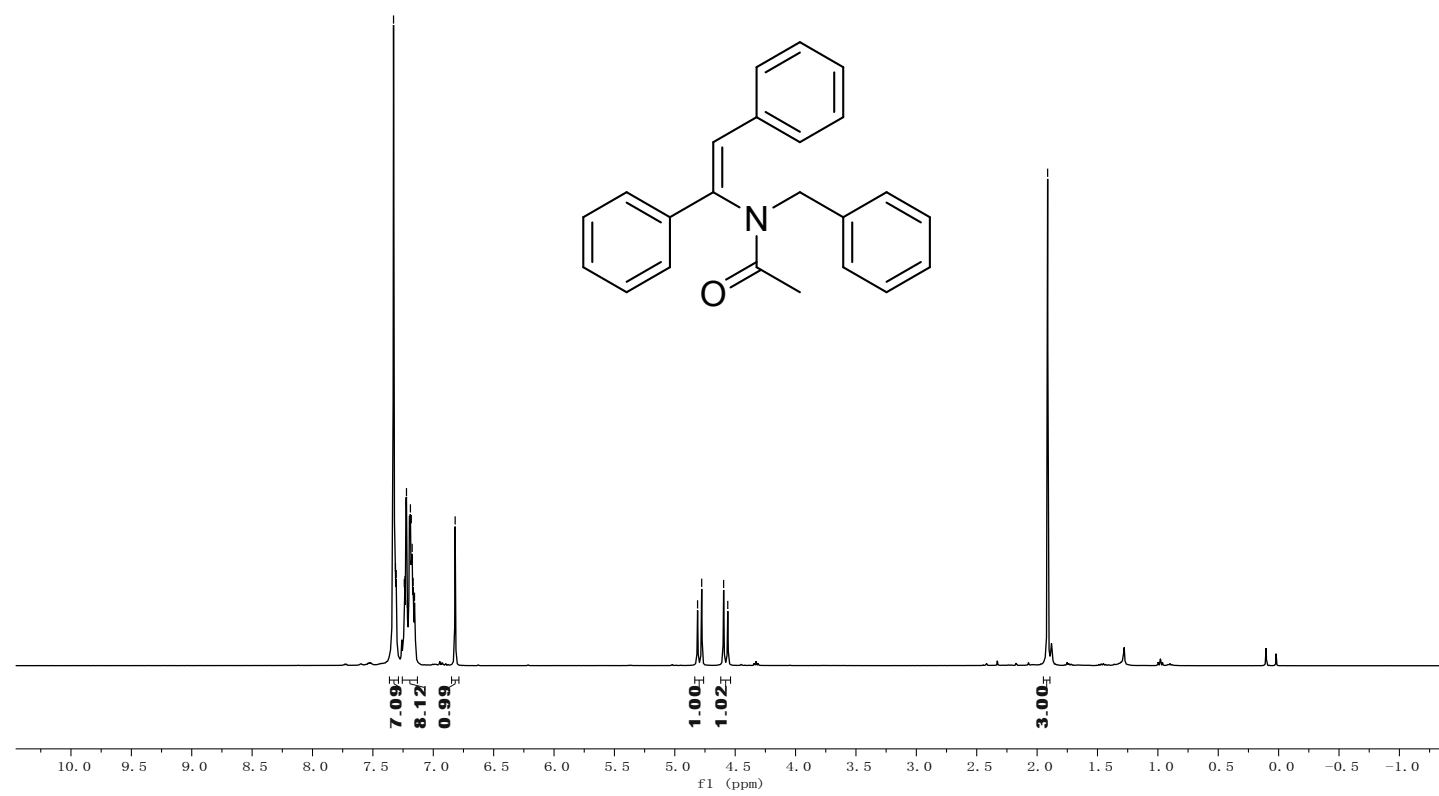

(Z)- $N$-benzyl- $N$-(1,2-diphenylvinyl)acetamide (3a)

${ }^{13} \mathrm{C}$ NMR (101 MHz, $\mathrm{CDCl}_{3}$ )

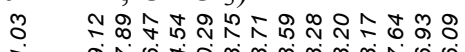

望
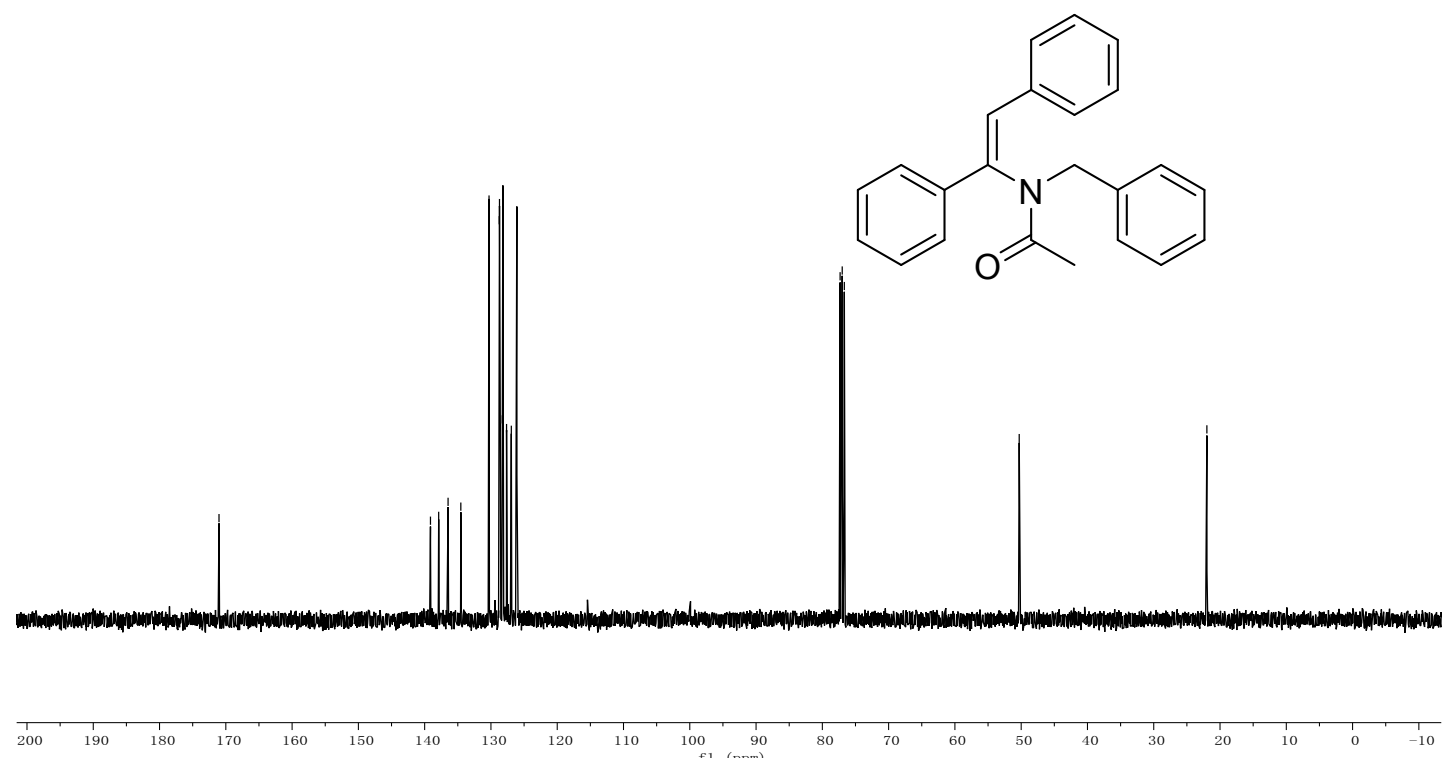

(Z)- $N$-benzyl- $N$-(2-phenyl-1-(p-tolyl)vinyl)acetamide (3b)

${ }^{1} \mathrm{H}$ NMR $\left(400 \mathrm{MHz}, \mathrm{CDCl}_{3}\right)$ 


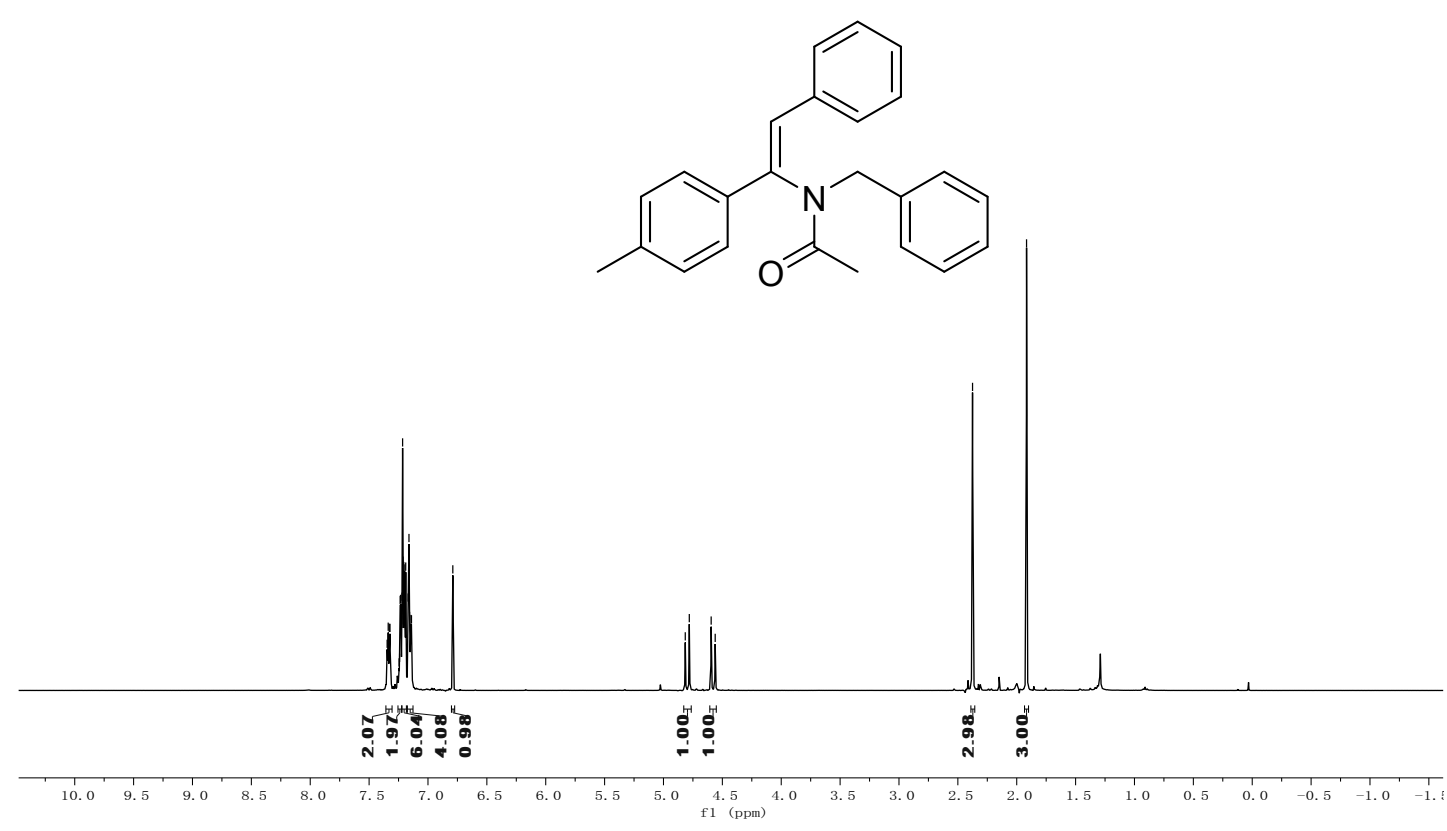

(Z)-N-benzyl- $N$-(2-phenyl-1-(p-tolyl)vinyl)acetamide (3b)

${ }^{13} \mathrm{C}$ NMR (101 MHz, $\left.\mathrm{CDCl}_{3}\right)$

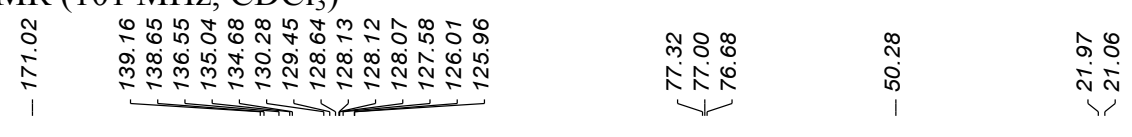
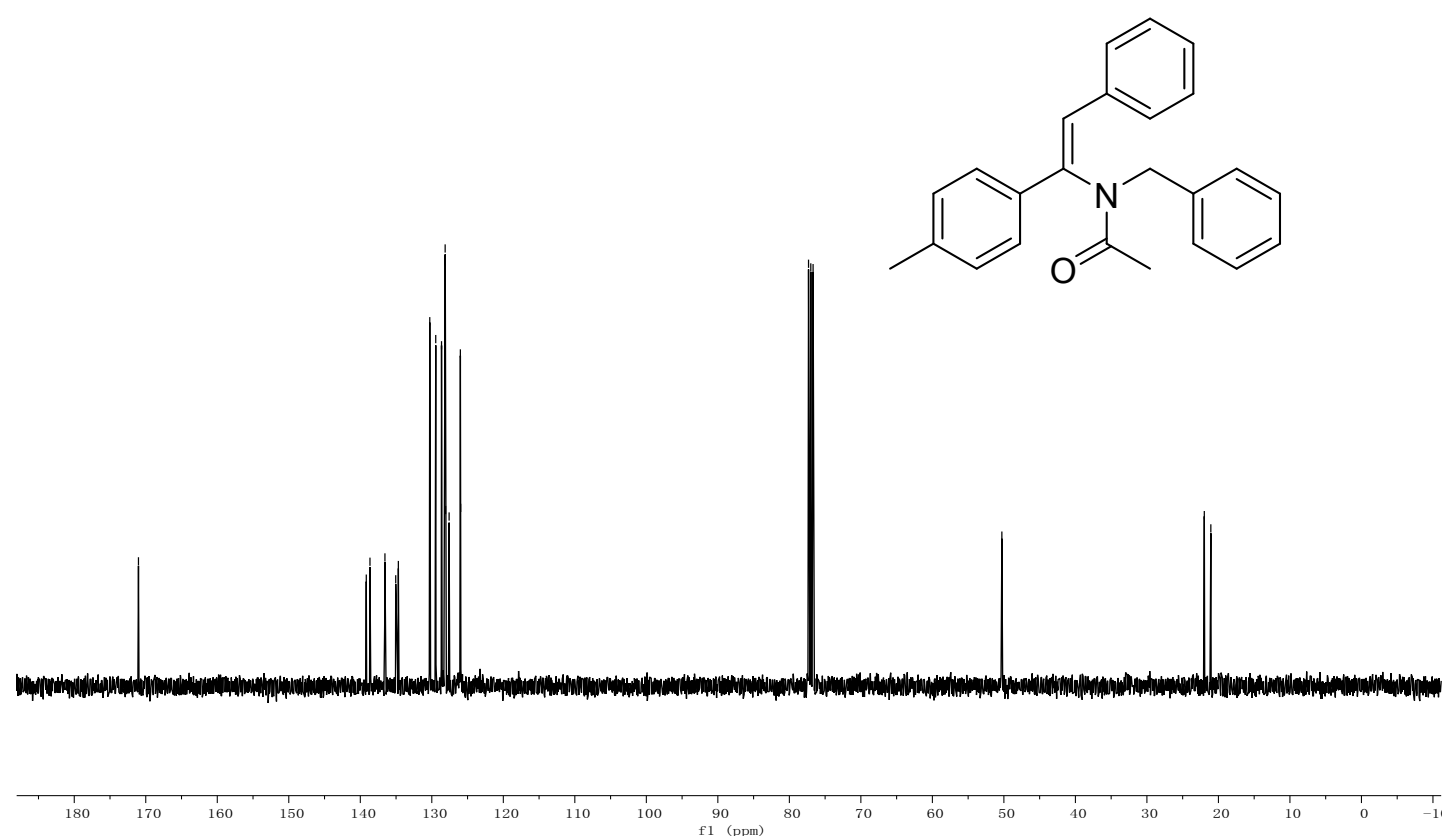

(Z)- $N$-benzyl- $N$-(1-(4-methoxyphenyl)-2-phenylvinyl)acetamide (3c)

${ }^{1} \mathrm{H} \mathrm{NMR}\left(400 \mathrm{MHz}, \mathrm{CDCl}_{3}\right)$ 
<smiles>COc1ccc(/C(=C/c2ccccc2)N(Cc2ccccc2)C(C)=O)cc1</smiles>

$$
\mathrm{MeO}
$$

(Z)- $N$-benzyl- $N$-(1-(4-methoxyphenyl)-2-phenylvinyl)acetamide (3c)

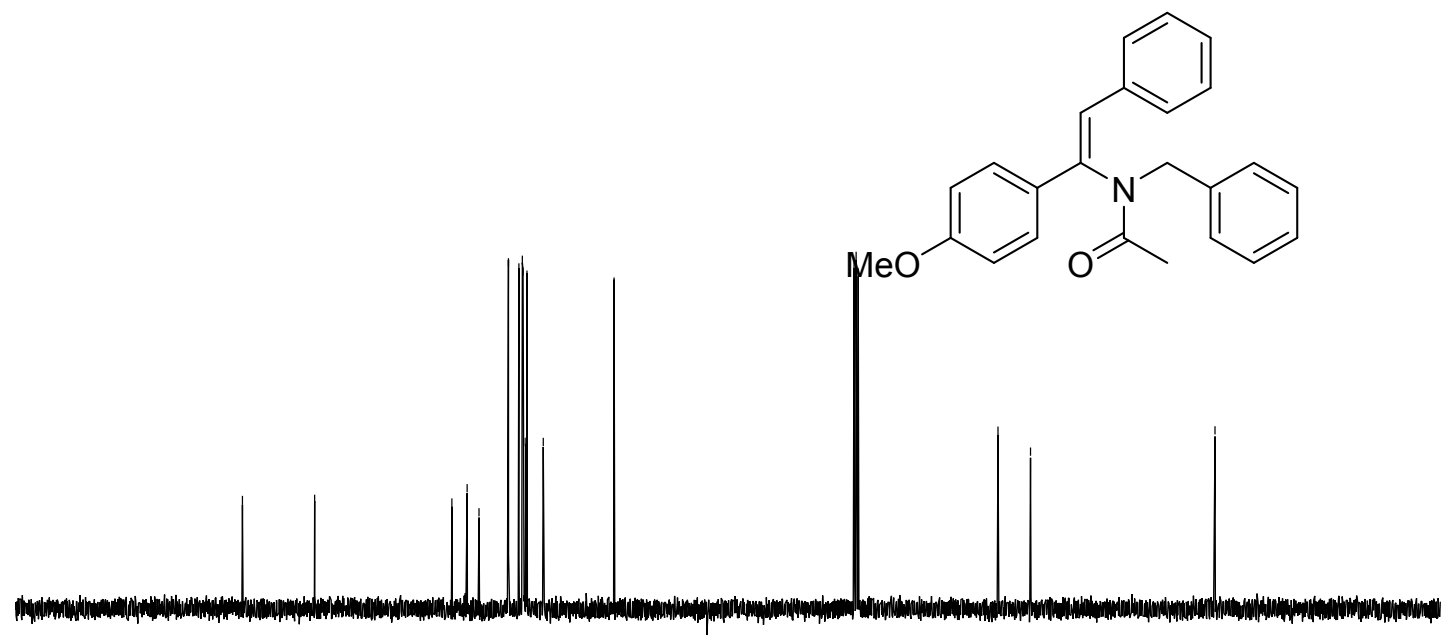

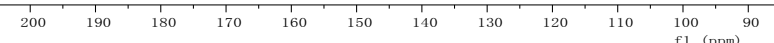

(Z)- $N$-benzyl- $N$-(1-(3-methoxyphenyl)-2-phenylvinyl)acetamide (3d)

${ }^{1} \mathrm{H}$ NMR (400 MHz, $\left.\mathrm{CDCl}_{3}\right)$ 


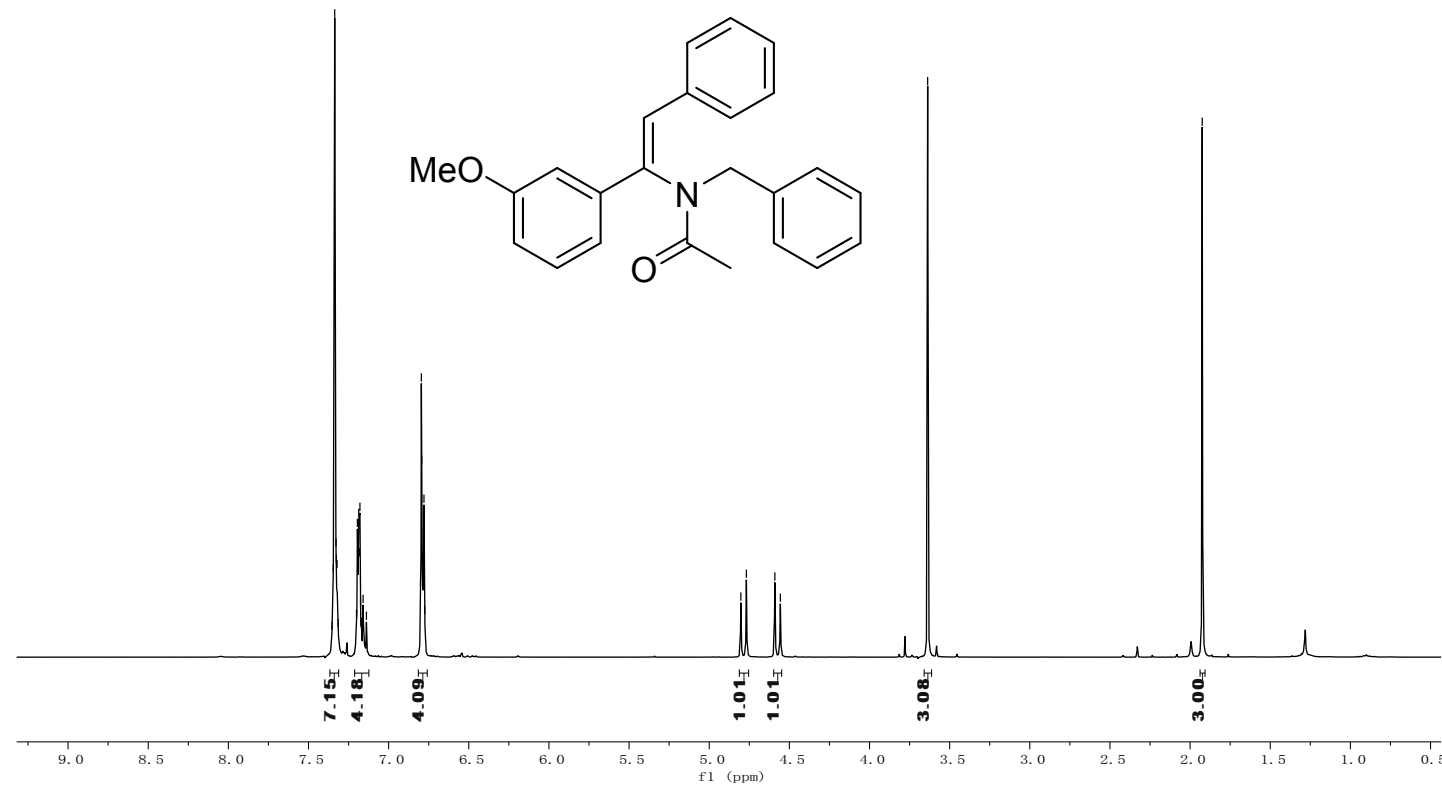

(Z)- $N$-benzyl- $N$-(1-(3-methoxyphenyl)-2-phenylvinyl)acetamide (3d) ${ }^{13} \mathrm{C}$ NMR $\left(101 \mathrm{MHz}, \mathrm{CDCl}_{3}\right)$

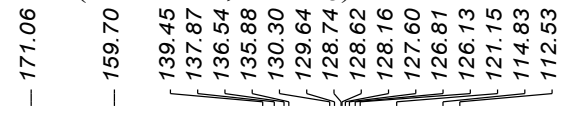

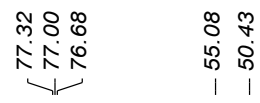

के

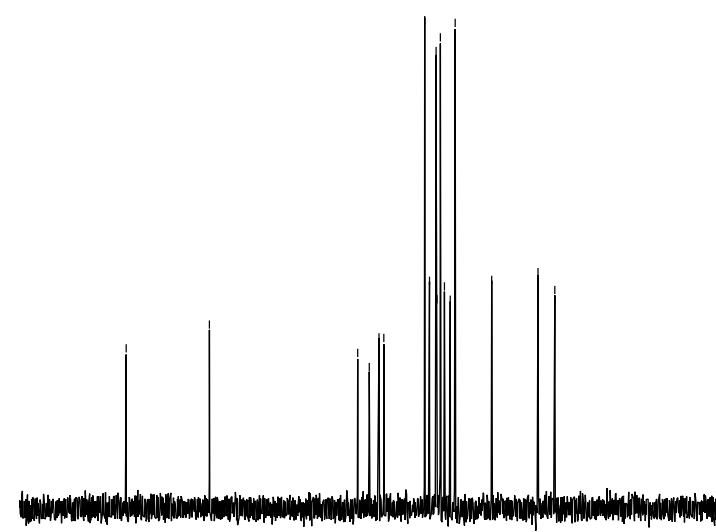<smiles>COc1cccc(/C(=C/c2ccccc2)N(Cc2ccccc2)C(C)=O)c1</smiles>

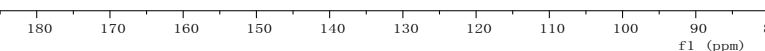

(Z)- $N$-benzyl- $N$-(1-(2-methoxyphenyl)-2-phenylvinyl)acetamide (3e)

${ }^{1} \mathrm{H} \mathrm{NMR}\left(400 \mathrm{MHz}, \mathrm{CDCl}_{3}\right)$ 


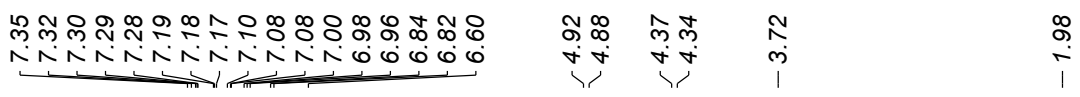<smiles>COc1ccccc1/C(=C/c1ccccc1)N(Cc1ccccc1)C(C)=O</smiles>

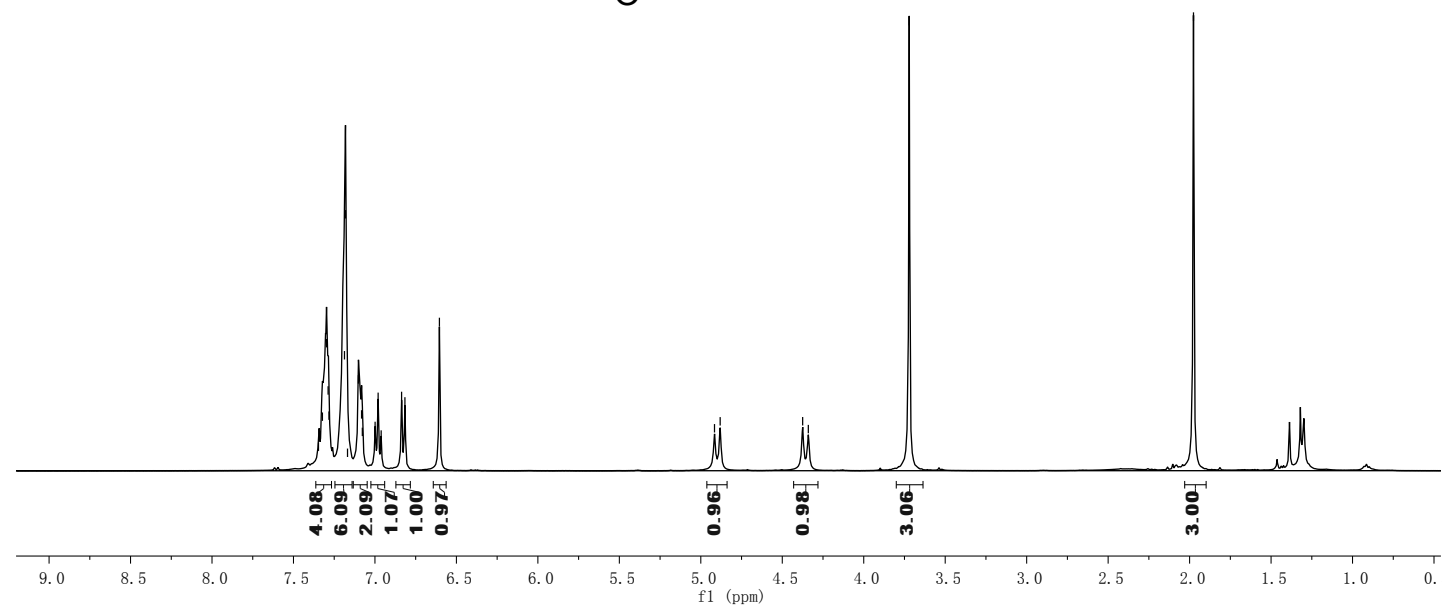

(Z)- $N$-benzyl- $N$-(1-(2-methoxyphenyl)-2-phenylvinyl)acetamide (3e)

${ }^{13} \mathrm{C}$ NMR $\left(101 \mathrm{MHz}, \mathrm{CDCl}_{3}\right)$

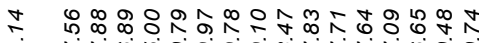

年

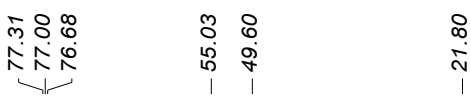

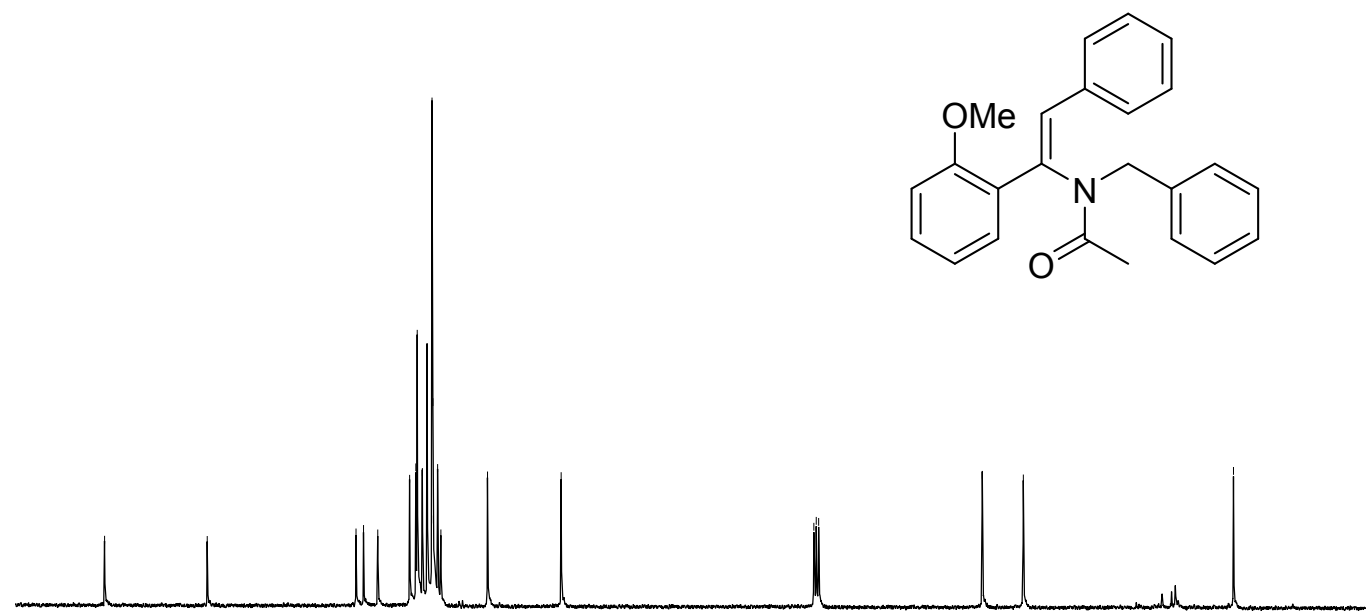

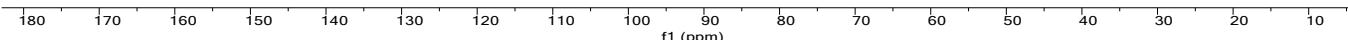

(Z)- $N$-benzyl- $N$-(1-(2,4-dimethoxyphenyl)-2-phenylvinyl)acetamide (3f)

${ }^{1} \mathrm{H}$ NMR $\left(400 \mathrm{MHz}, \mathrm{CDCl}_{3}\right)$ 


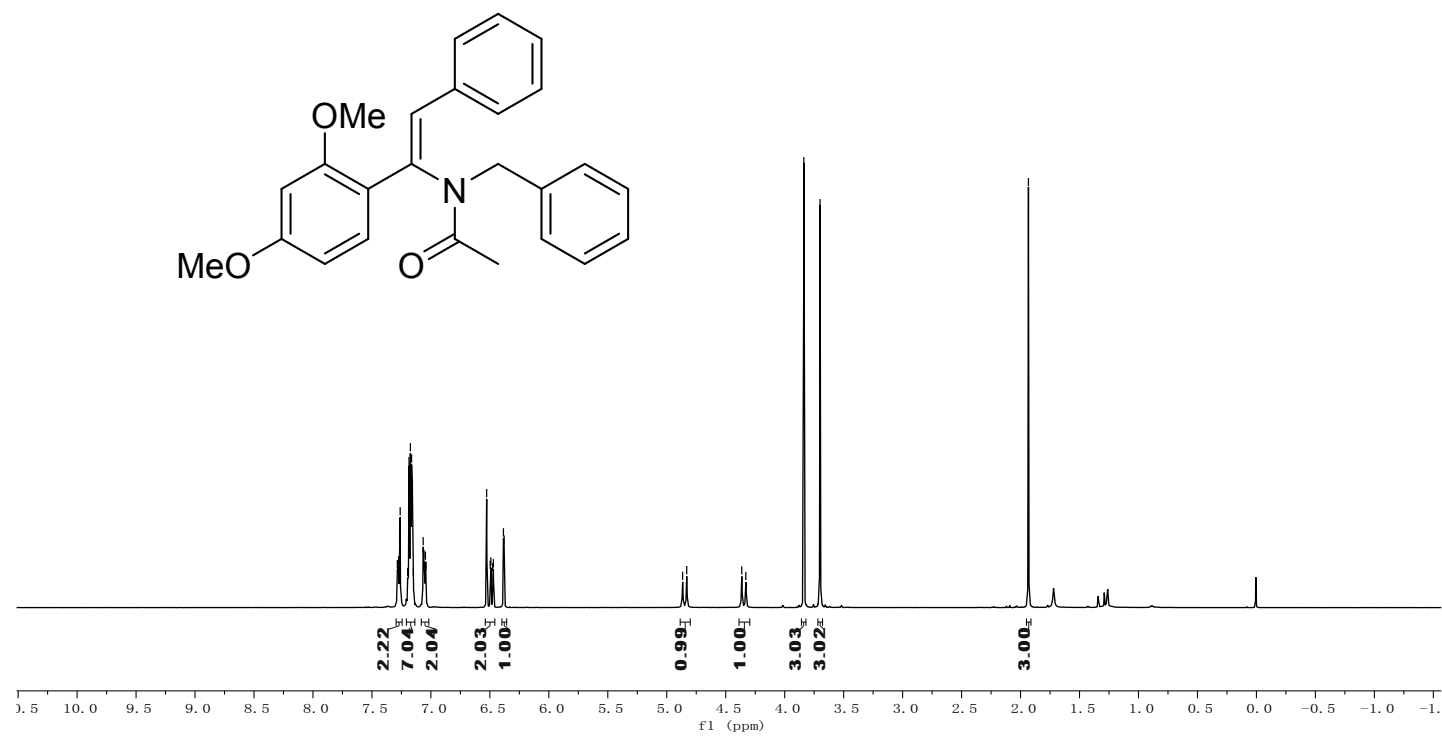

(Z)- $N$-benzyl- $N$-(1-(2,4-dimethoxyphenyl)-2-phenylvinyl)acetamide (3f) ${ }^{13} \mathrm{C}$ NMR $\left(101 \mathrm{MHz}, \mathrm{CDCl}_{3}\right)$

mos

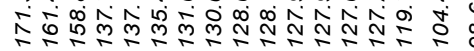

mo $8:$

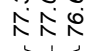

$\forall \stackrel{\sim}{\sim} \stackrel{\infty}{\stackrel{\infty}{\infty}}$

它综字

8

少

ลั.<smiles>COc1ccc(/C(=C/c2ccccc2)N(Cc2ccccc2)C(C)=O)c(OC)c1</smiles>

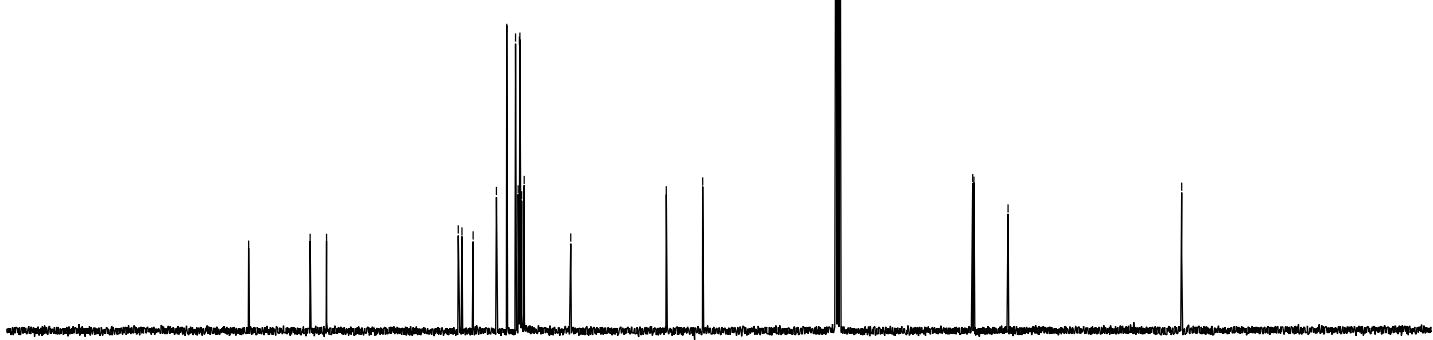

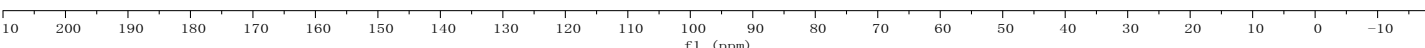

(Z)- $N$-benzyl- $N$-(2-phenyl-1-(3,4,5-trimethoxyphenyl)vinyl)acetamide (3g) ${ }^{1} \mathrm{H}$ NMR $\left(400 \mathrm{MHz}, \mathrm{CDCl}_{3}\right)$ 


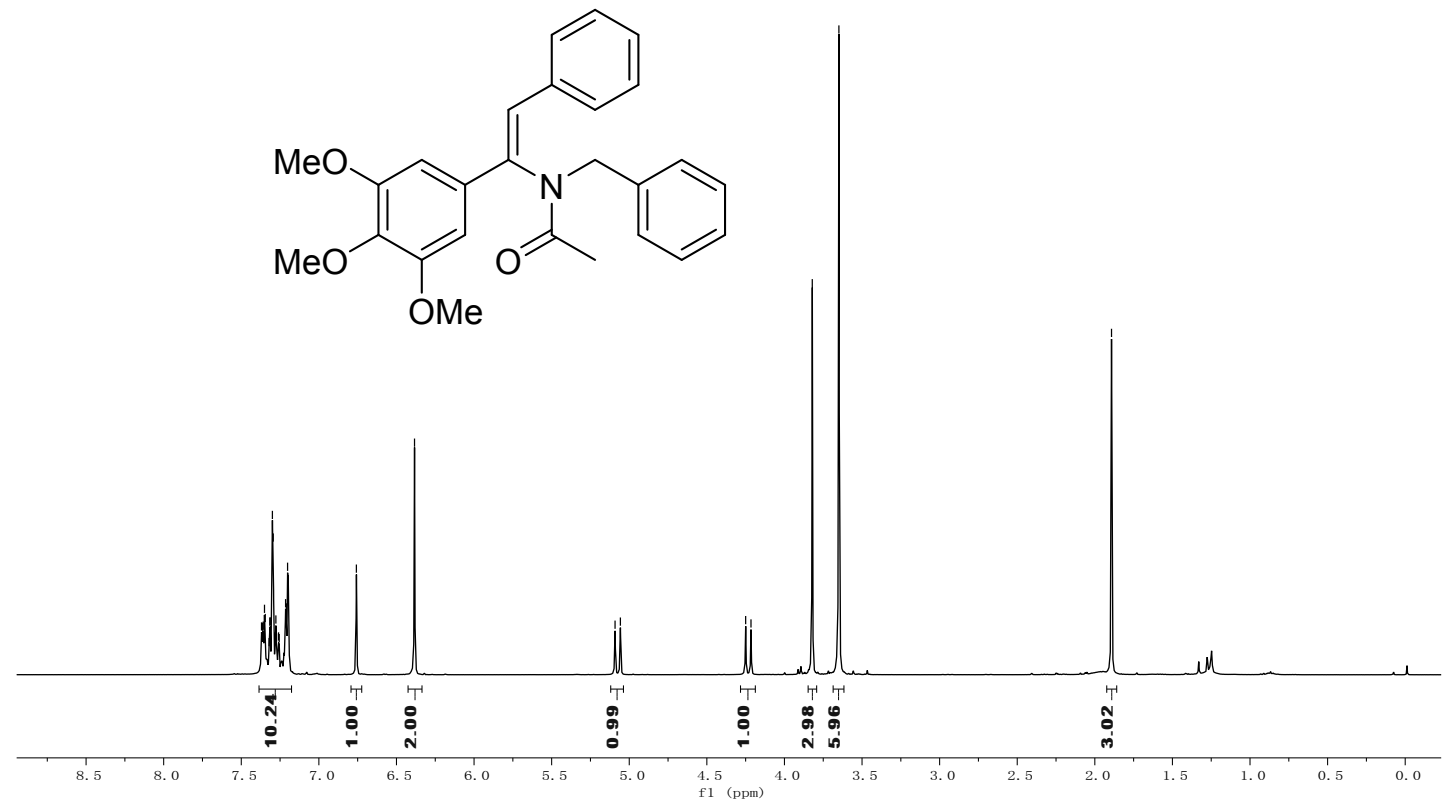

(Z)- $N$-benzyl- $N$-(2-phenyl-1-(3,4,5-trimethoxyphenyl)vinyl)acetamide (3g) ${ }^{13} \mathrm{C}$ NMR (101 MHz, $\left.\mathrm{CDCl}_{3}\right)$

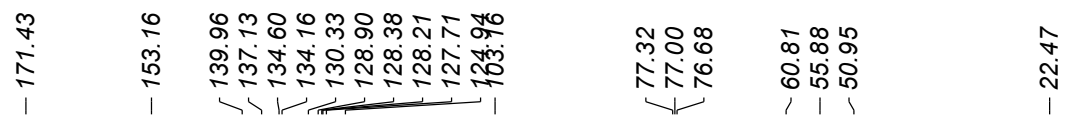

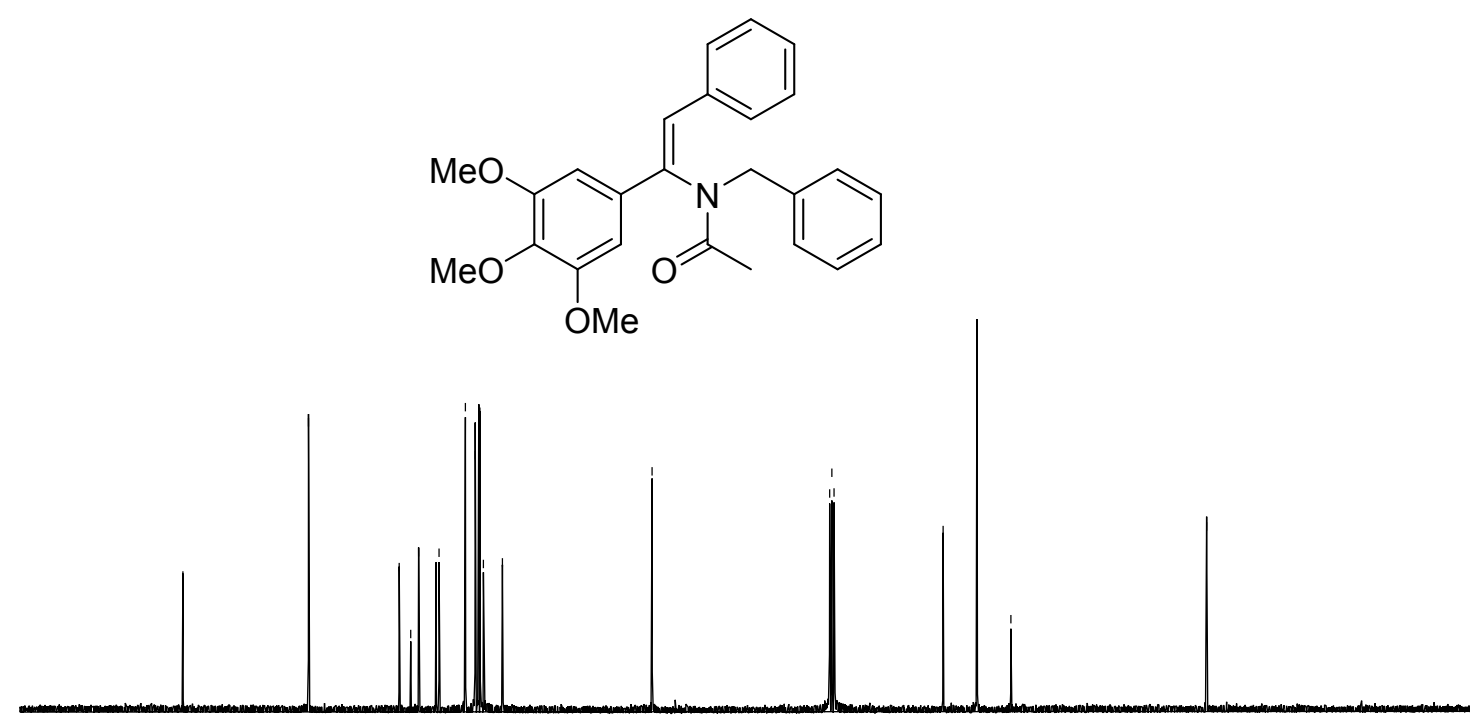

(Z)- $N$-(1-(benzo[d][1,3]dioxol-5-yl)-2-phenylvinyl)- $N$-benzylacetamide (3h)

${ }^{1} \mathrm{H}$ NMR (400 MHz, $\mathrm{CDCl}_{3}$ ) 
<smiles>CC(=O)N(Cc1ccccc1)/C(=C\c1ccccc1)c1ccc2c(c1)OCO2</smiles>

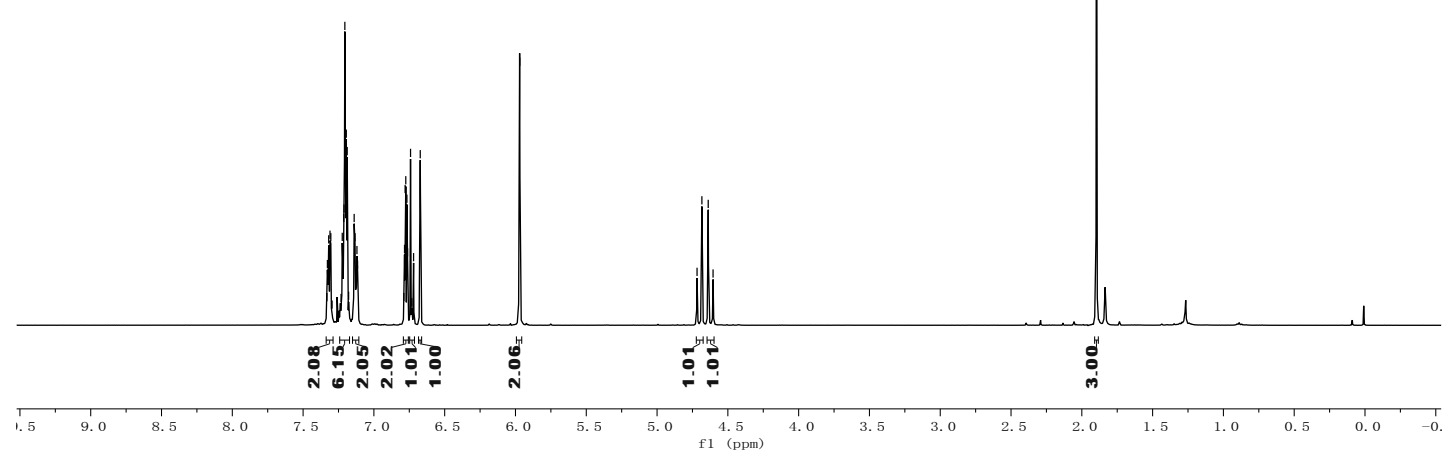

(Z)- $N$-(1-(benzo[d][1,3]dioxol-5-yl)-2-phenylvinyl)- $N$-benzylacetamide (3h)

\section{${ }^{13} \mathrm{C} \mathrm{NMR}\left(101 \mathrm{MHz}, \mathrm{CDCl}_{3}\right)$}

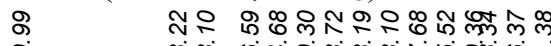

ลे

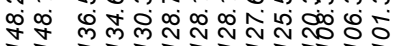

年

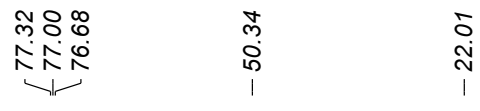<smiles>CC(=O)N(Cc1ccccc1)/C(=C\c1ccccc1)c1ccc2c(c1)OCO2</smiles>
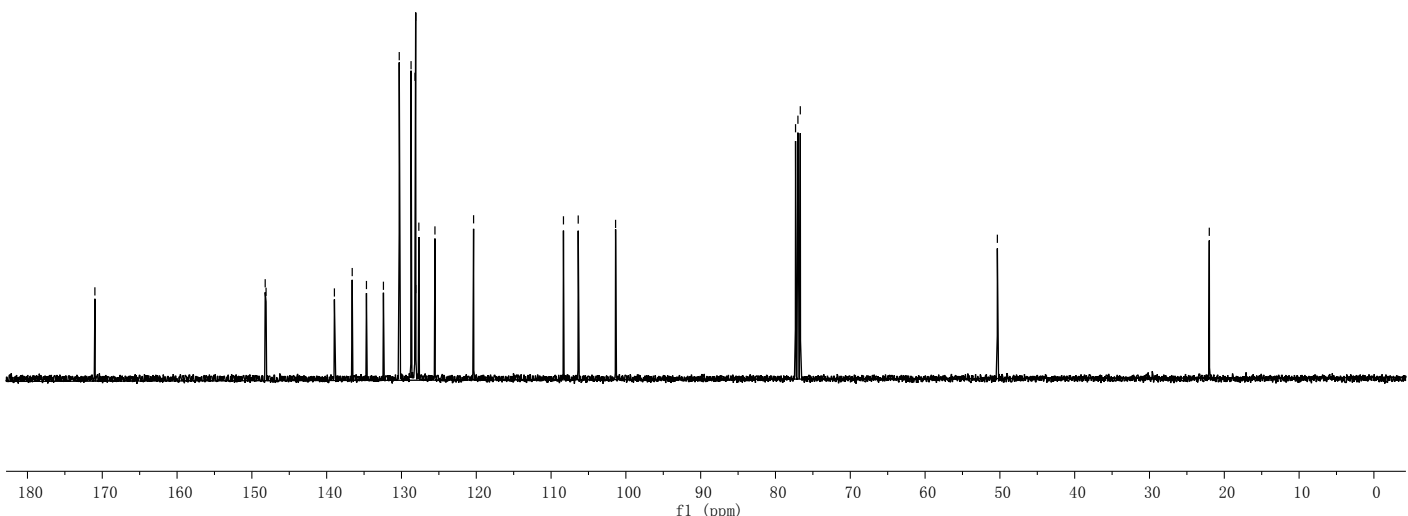

(Z)- $N$-benzyl- $N$-(1-(4-fluorophenyl)-2-phenylvinyl)acetamide (3i)

${ }^{1} \mathrm{H}$ NMR $\left(400 \mathrm{MHz}, \mathrm{CDCl}_{3}\right)$ 
<smiles>CC(=O)N(Cc1ccccc1)/C(=C\c1ccccc1)c1ccc(F)cc1</smiles>

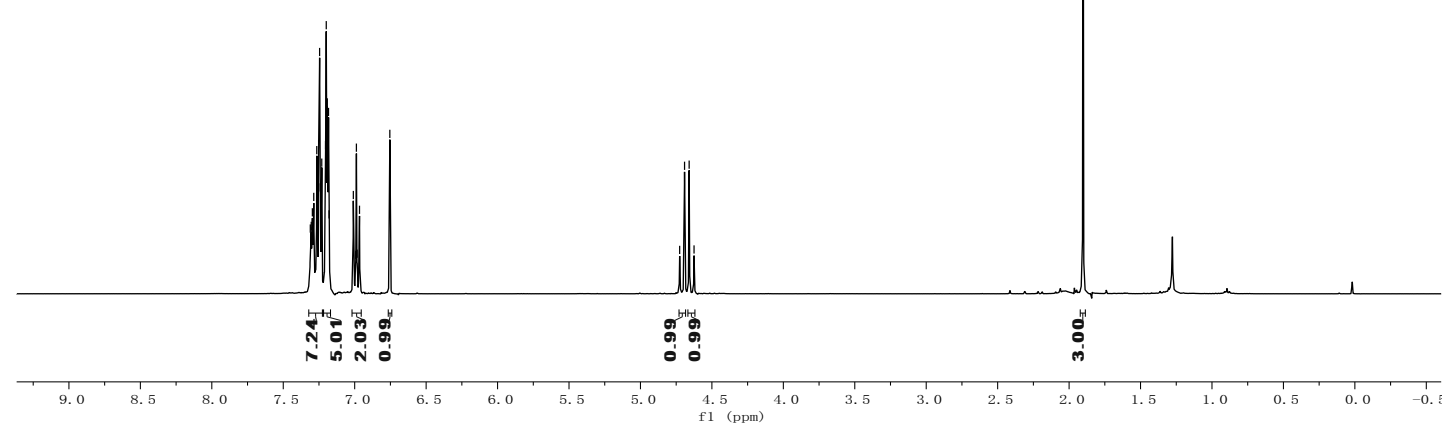

(Z)- $N$-benzyl- $N$-(1-(4-fluorophenyl)-2-phenylvinyl)acetamide (3i)

${ }^{13} \mathrm{C}$ NMR (101 MHz, $\mathrm{CDCl}_{3}$ )

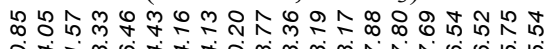

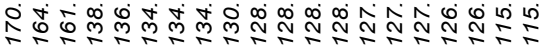

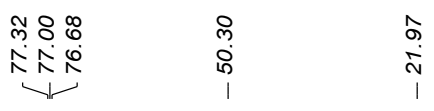

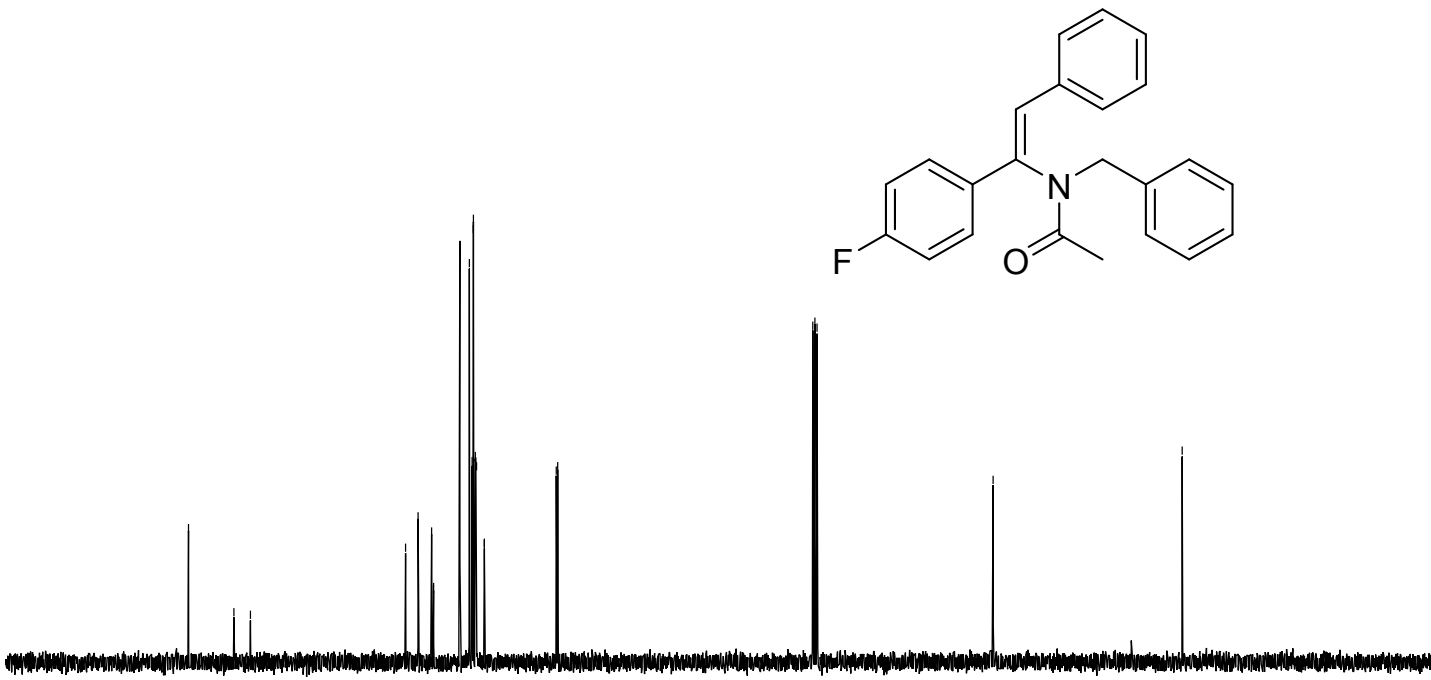

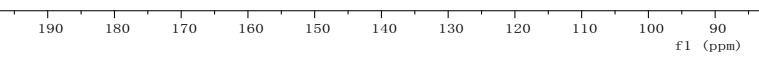

(Z)- $N$-benzyl- $N$-(1-(4-chlorophenyl)-2-phenylvinyl)acetamide (3j)

${ }^{1} \mathrm{H}$ NMR (400 MHz, $\mathrm{CDCl}_{3}$ ) 
<smiles>CC(=O)N(Cc1ccccc1)/C(=C\c1ccccc1)c1ccc(Cl)cc1</smiles>

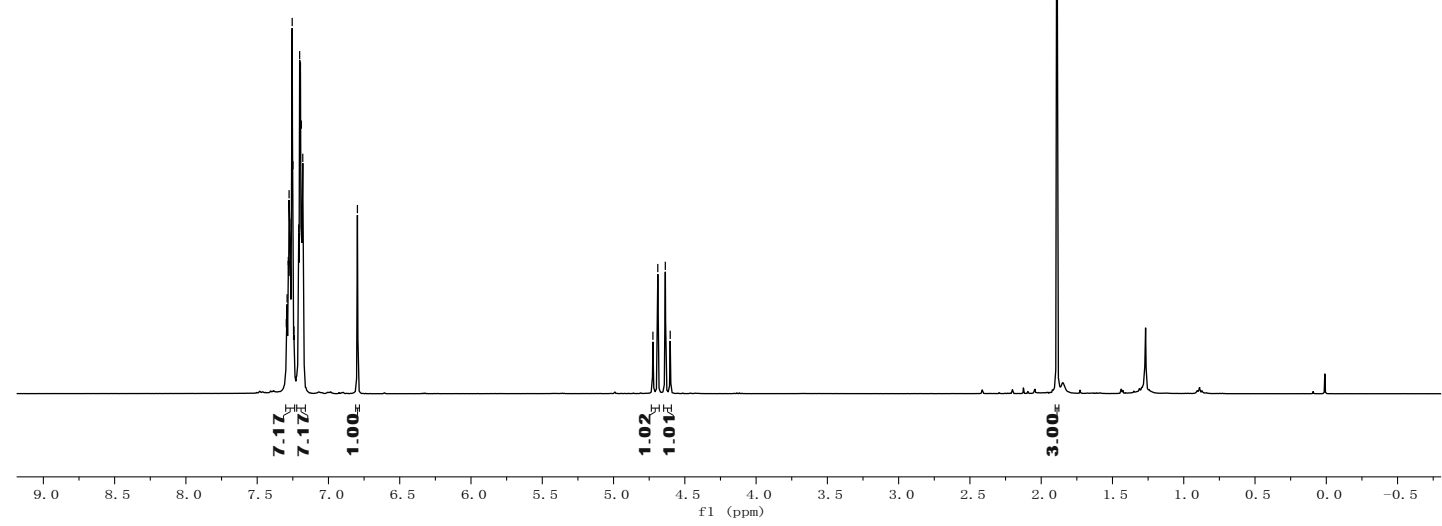

(Z)- $N$-benzyl- $N$-(1-(4-chlorophenyl)-2-phenylvinyl)acetamide (3j) ${ }^{13} \mathrm{C}$ NMR (101 MHz, $\mathrm{CDCl}_{3}$ )

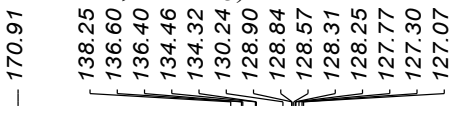

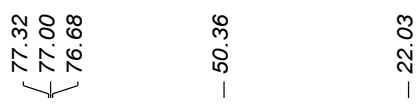

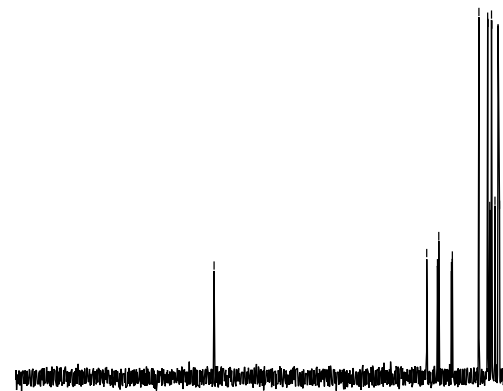<smiles>CC(=O)N(Cc1ccccc1)/C(=C\c1ccccc1)c1ccc(Cl)cc1</smiles>

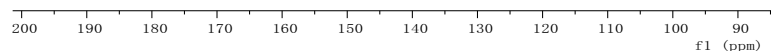

(Z)- $N$-benzyl- $N$-(1-(4-bromophenyl)-2-phenylvinyl)acetamide (3k) ${ }^{1} \mathrm{H}$ NMR $\left(400 \mathrm{MHz}, \mathrm{CDCl}_{3}\right)$ 
<smiles>CC(=O)N(Cc1ccccc1)/C(=C\c1ccccc1)c1ccc(Br)cc1</smiles>

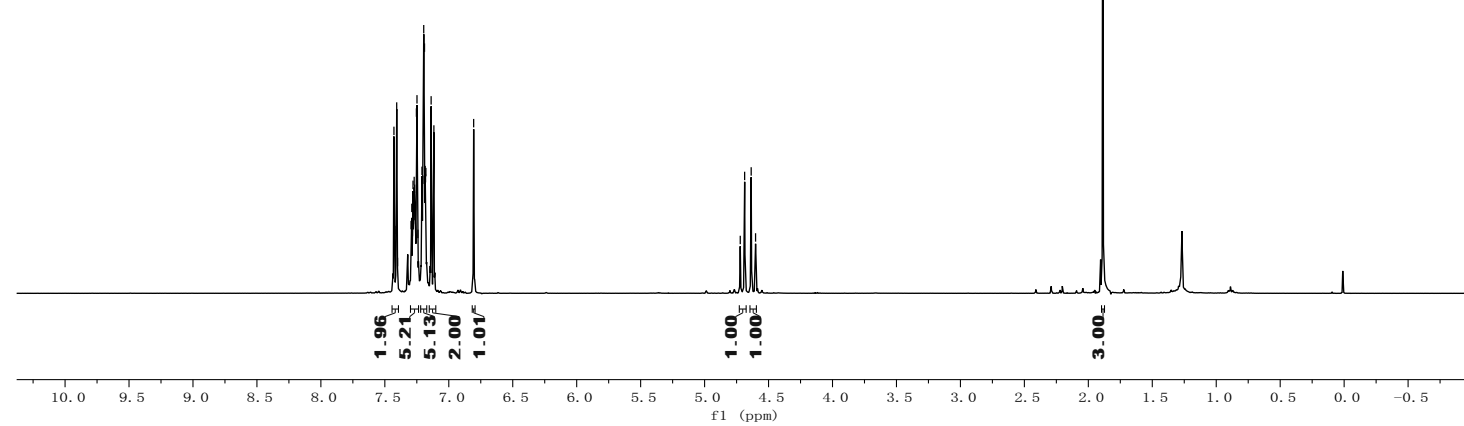

(Z)- $N$-benzyl- $N$-(1-(4-bromophenyl)-2-phenylvinyl)acetamide (3k)

${ }^{13} \mathrm{C}$ NMR (101 MHz, $\mathrm{CDCl}_{3}$ )

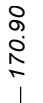

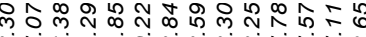

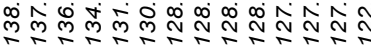

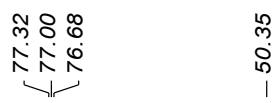

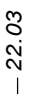

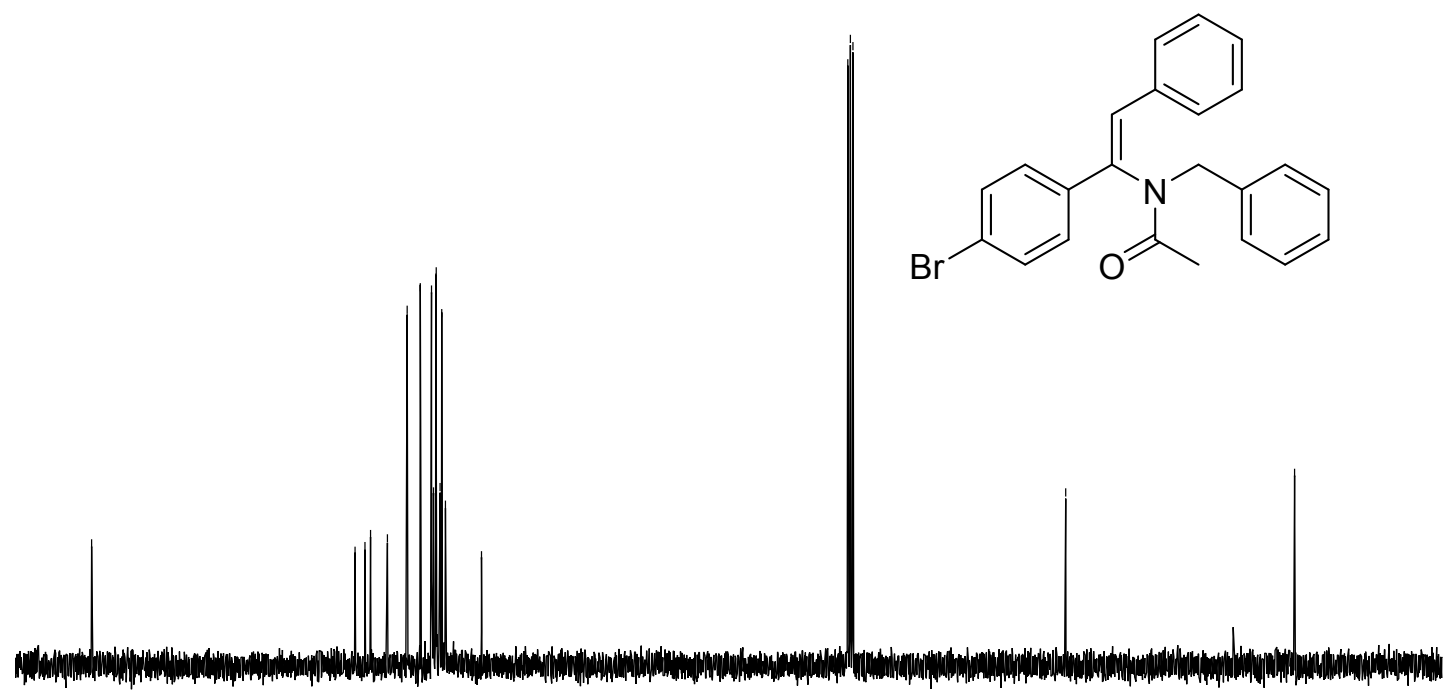

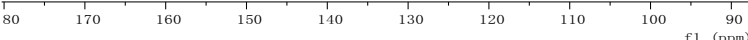

(Z)- $N$-benzyl- $N$-(1-(3-bromophenyl)-2-phenylvinyl)acetamide (3I)

${ }^{1} \mathrm{H}$ NMR $\left(400 \mathrm{MHz}, \mathrm{CDCl}_{3}\right)$ 
<smiles>CC(=O)N(Cc1ccccc1)/C(=C\c1ccccc1)c1cccc(Br)c1</smiles>

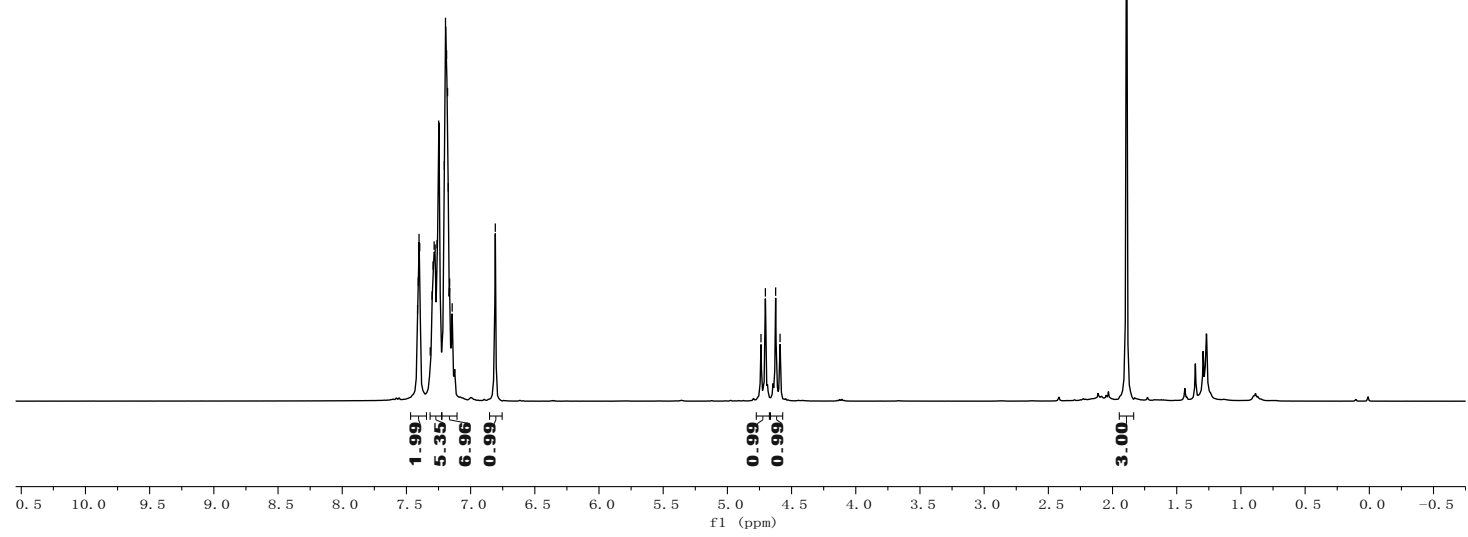

(Z)- $N$-benzyl- $N$-(1-(3-bromophenyl)-2-phenylvinyl)acetamide (3I) ${ }^{13} \mathrm{C}$ NMR (101 MHz, $\mathrm{CDCl}_{3}$ )

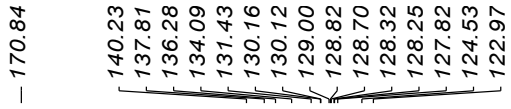

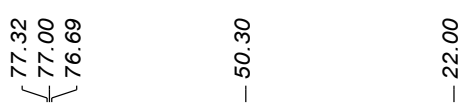

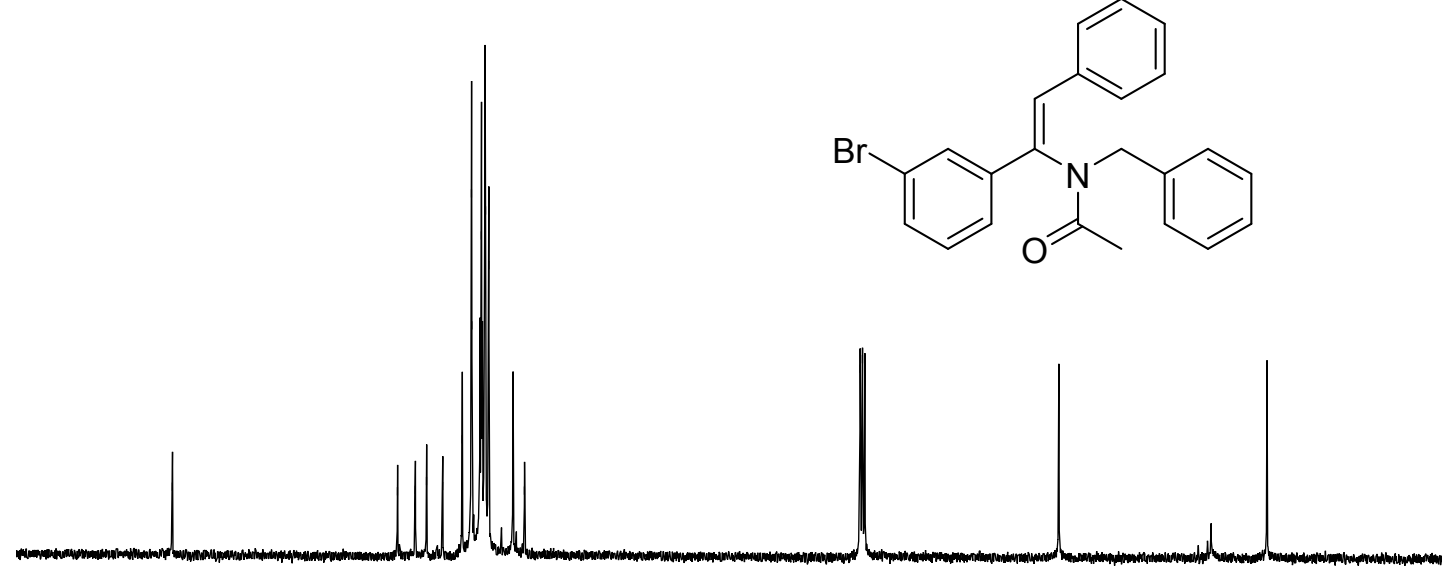

(Z)- $N$-benzyl- $N$-(1-(2-bromophenyl)-2-phenylvinyl)acetamide (3m) ${ }^{1} \mathrm{H}$ NMR $\left(400 \mathrm{MHz}, \mathrm{CDCl}_{3}\right)$ 
<smiles>CC(=O)N(Cc1ccccc1)/C(=C\c1ccccc1)c1ccccc1Br</smiles>

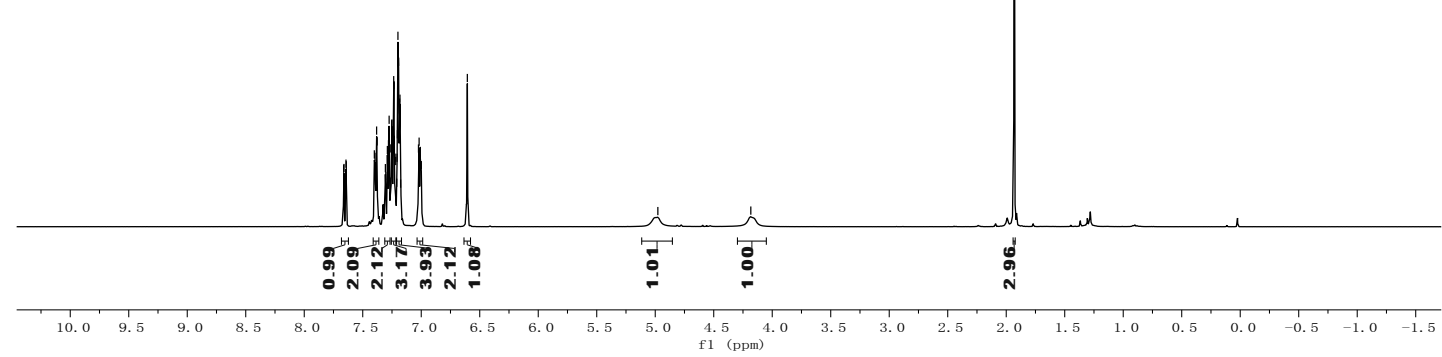

(Z)-N-benzyl-N-(1-(2-bromophenyl)-2-phenylvinyl)acetamide (3 m) ${ }^{13} \mathrm{C}$ NMR $\left(101 \mathrm{MHz}, \mathrm{CDCl}_{3}\right)$

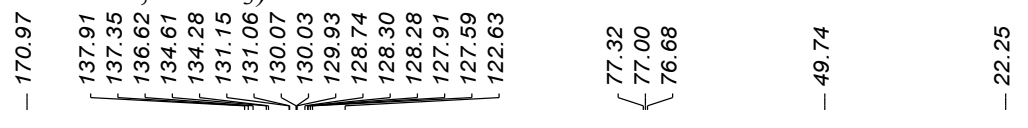

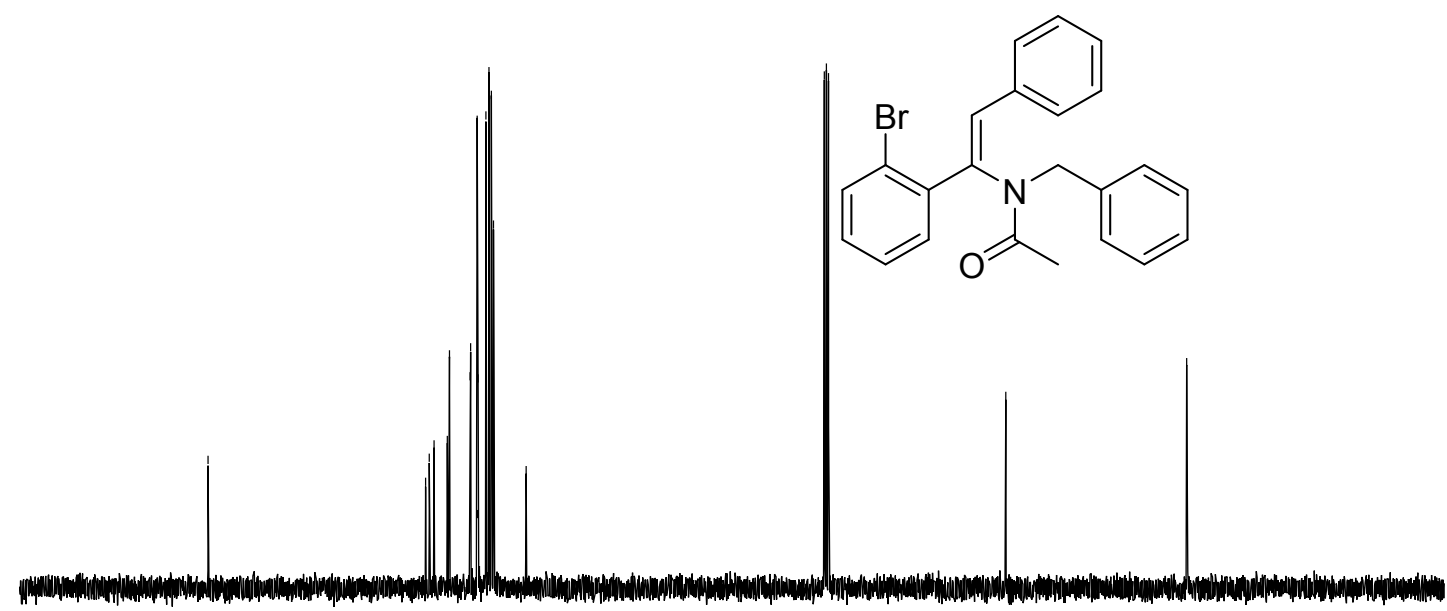

(Z)- $N$-benzyl- $N$-(2-phenyl-1-(4-(trifluoromethyl)phenyl)vinyl)acetamide (3n) ${ }^{1} \mathrm{H}$ NMR $\left(400 \mathrm{MHz}, \mathrm{CDCl}_{3}\right)$ 
<smiles>CC(=O)N(Cc1ccccc1)/C(=C\c1ccccc1)c1ccc(C(F)(F)F)cc1</smiles>

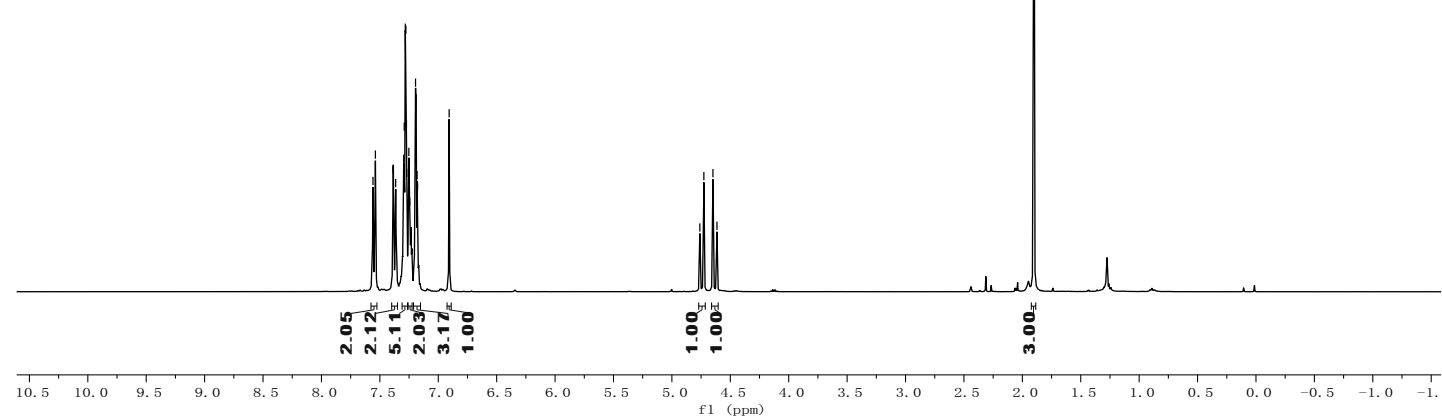

(Z)- $N$-benzyl- $N$-(2-phenyl-1-(4-(trifluoromethyl)phenyl)vinyl)acetamide (3n) ${ }^{13} \mathrm{C}$ NMR $\left(101 \mathrm{MHz}, \mathrm{CDCl}_{3}\right)$

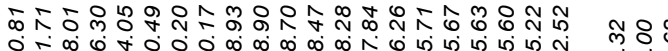

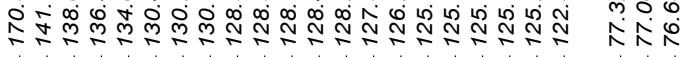

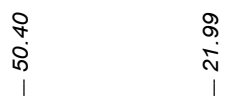
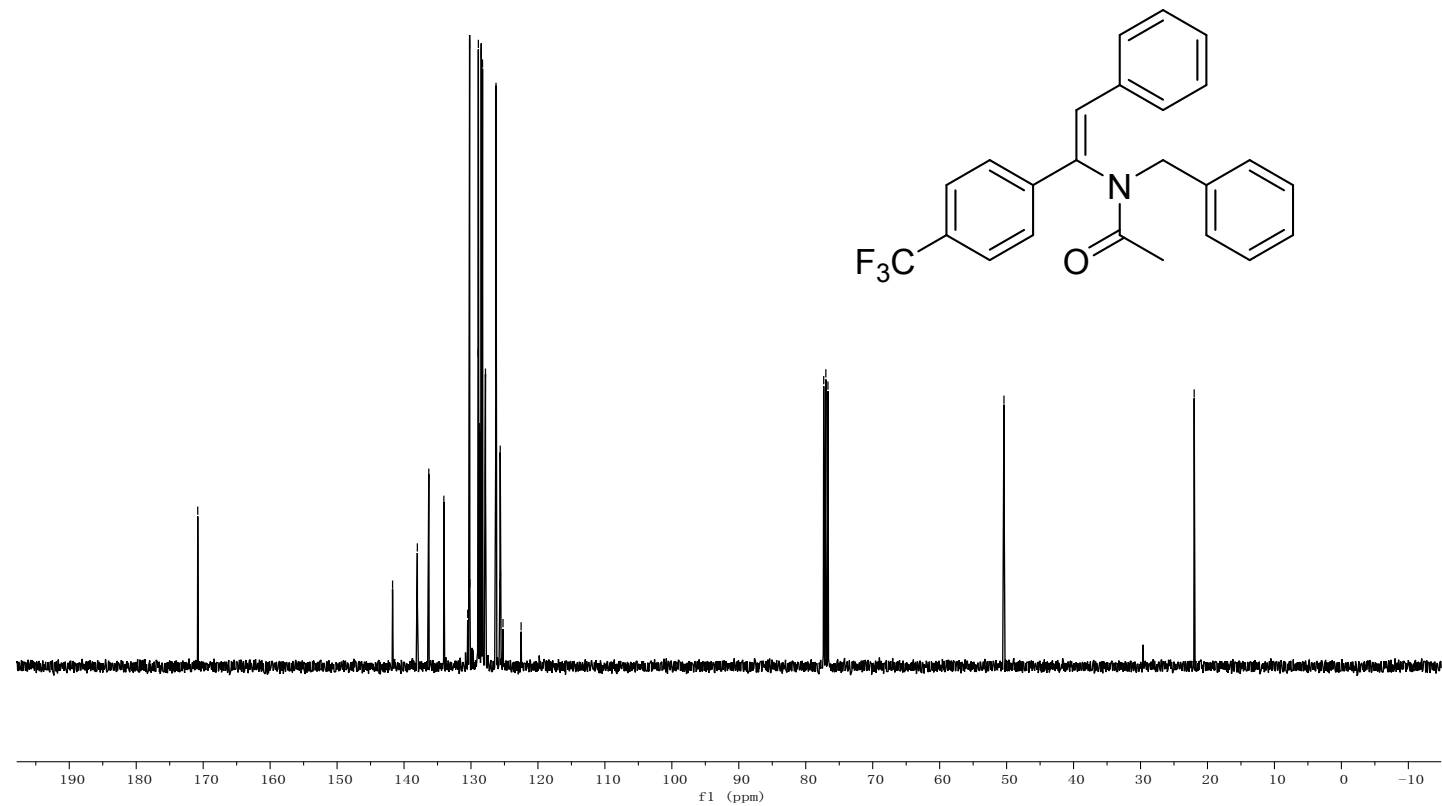

(Z)- $N$-benzyl- $N$-(1-(4-cyanophenyl)-2-phenylvinyl)acetamide (3o) ${ }^{1} \mathrm{H}$ NMR $\left(400 \mathrm{MHz}, \mathrm{CDCl}_{3}\right)$ 


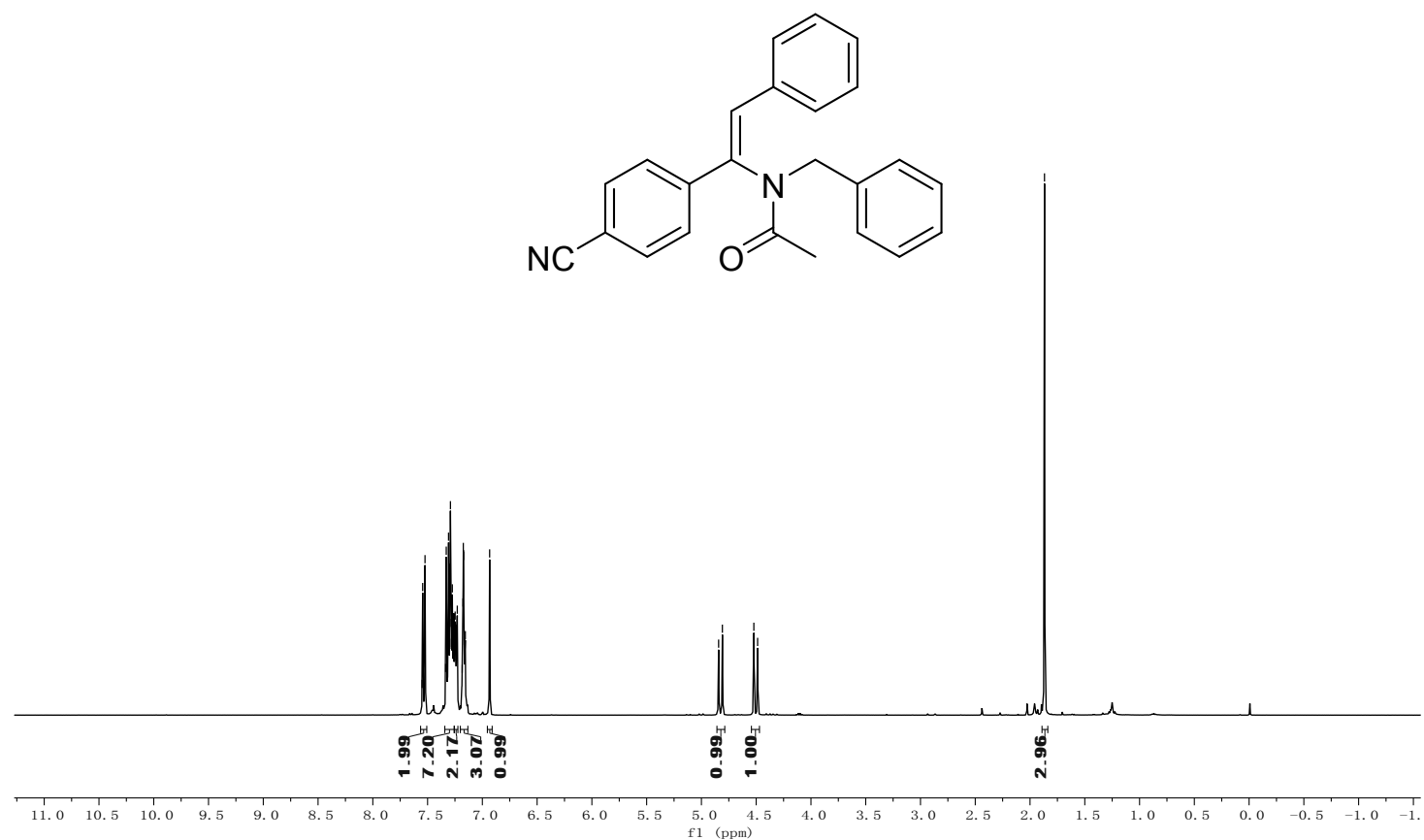

(Z)-N-benzyl- $N$-(1-(4-cyanophenyl)-2-phenylvinyl)acetamide (3o)

${ }^{13} \mathrm{C}$ NMR (101 MHz, $\left.\mathrm{CDCl}_{3}\right)$

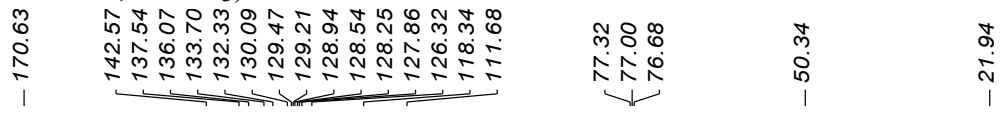

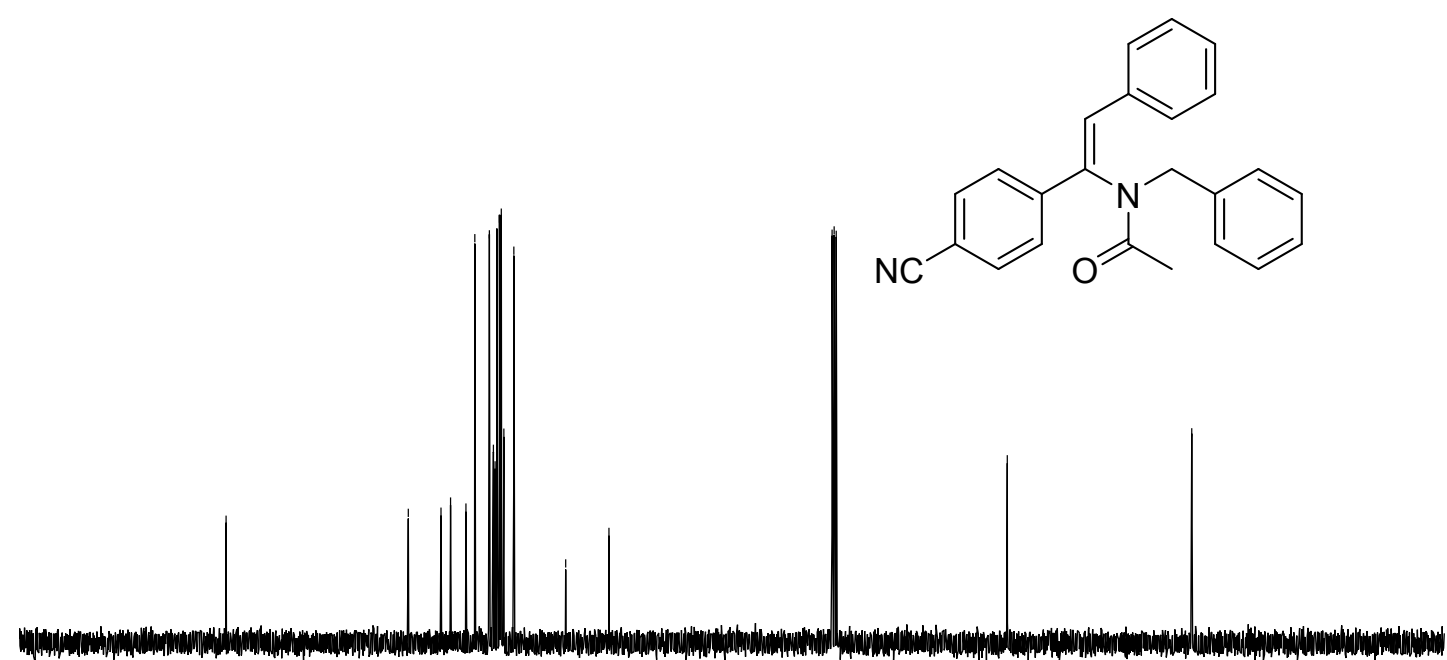

(Z)-N-benzyl- $N$-(1-(4-nitrophenyl)-2-phenylvinyl)acetamide (3p)

${ }^{1} \mathrm{H}$ NMR $\left(400 \mathrm{MHz}, \mathrm{CDCl}_{3}\right)$ 
요 \&

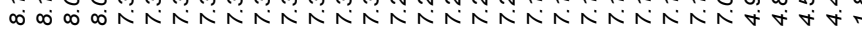<smiles>CC(=O)N(Cc1ccccc1)/C(=C\c1ccccc1)c1ccc([N+](=O)[O-])cc1</smiles>

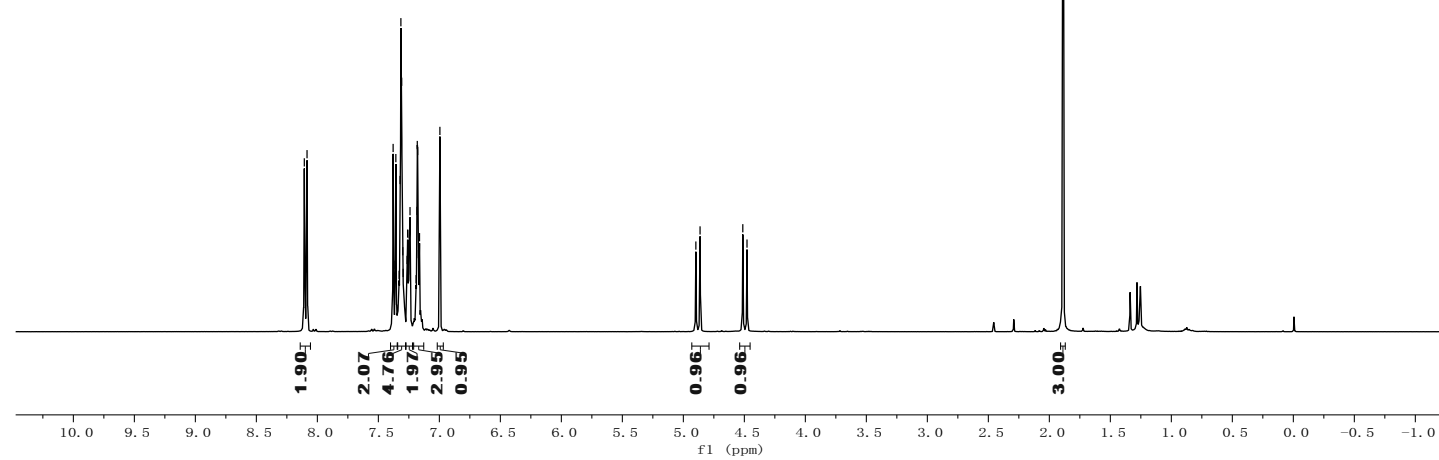

(Z)- $N$-benzyl- $N$-(1-(4-nitrophenyl)-2-phenylvinyl)acetamide (3p) ${ }^{13} \mathrm{C}$ NMR $\left(101 \mathrm{MHz}, \mathrm{CDCl}_{3}\right)$

ㄴ.

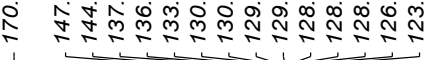

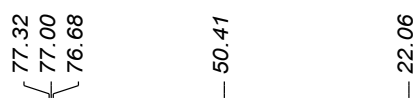

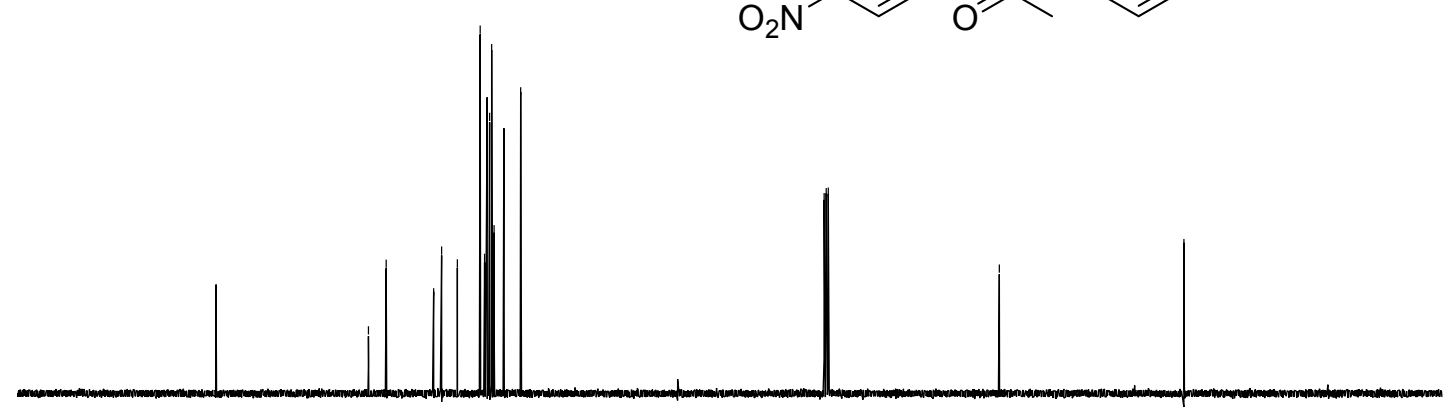<smiles>CC(=O)N(Cc1ccccc1)/C(=C\c1ccccc1)c1ccc([N+](=O)[O-])cc1</smiles>

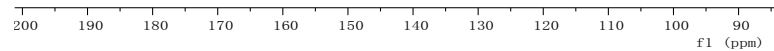

(Z)-N-(1-([1,1'-biphenyl]-4-yl)-2-phenylvinyl)- $N$-benzylacetamide (3q)

${ }^{1} \mathrm{H}$ NMR $\left(400 \mathrm{MHz}, \mathrm{CDCl}_{3}\right)$ 
<smiles>CC(=O)N(Cc1ccccc1)/C(=C\c1ccccc1)c1ccc(-c2ccccc2)cc1</smiles>

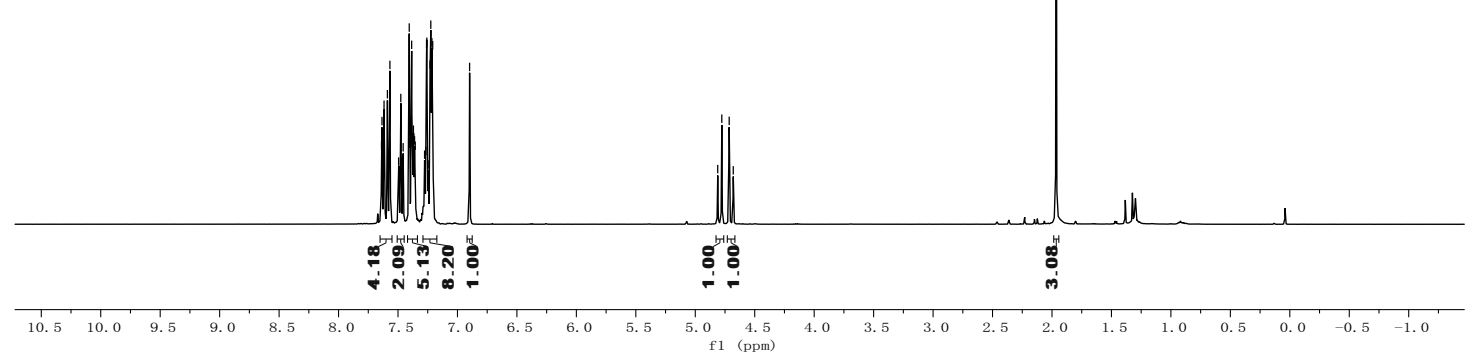

(Z)- $N$-(1-([1,1'-biphenyl]-4-yl)-2-phenylvinyl)- $N$-benzylacetamide (3q) ${ }^{13} \mathrm{C}$ NMR $\left(101 \mathrm{MHz}, \mathrm{CDCl}_{3}\right)$

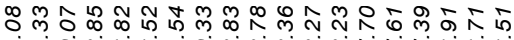

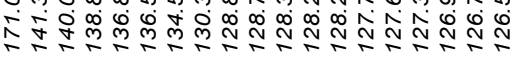

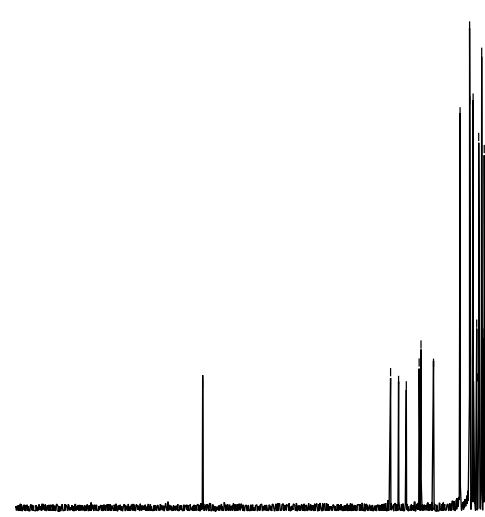<smiles>CC(=O)N(Cc1ccccc1)C(=Cc1ccccc1)c1ccc(-c2ccccc2)cc1</smiles>

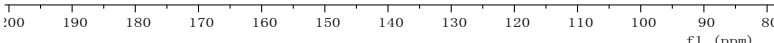

(Z)- $N$-benzyl- $N$-(1-phenylprop-1-en-2-yl)acetamide (3r) ${ }^{1} \mathrm{H}$ NMR $\left(400 \mathrm{MHz}, \mathrm{CDCl}_{3}\right)$ 
<smiles>CC(=O)N(Cc1ccccc1)/C(C)=C\c1ccccc1</smiles>

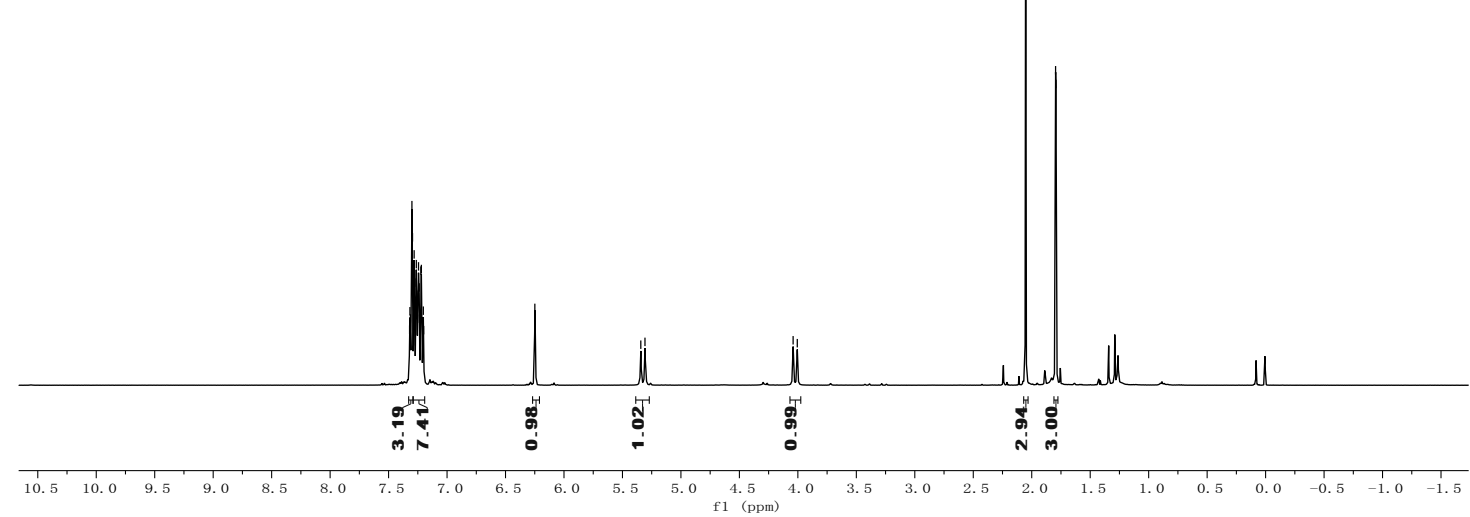

(Z)- $N$-benzyl- $N$-(1-phenylprop-1-en-2-yl)acetamide (3r) ${ }^{13} \mathrm{C}$ NMR (101 MHz, $\mathrm{CDCl}_{3}$ )

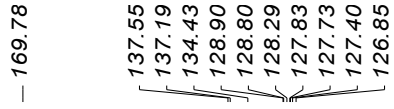

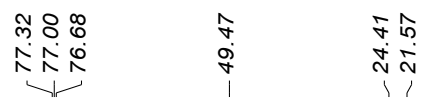<smiles>CC(=O)N(Cc1ccccc1)/C(C)=C\c1ccccc1</smiles>

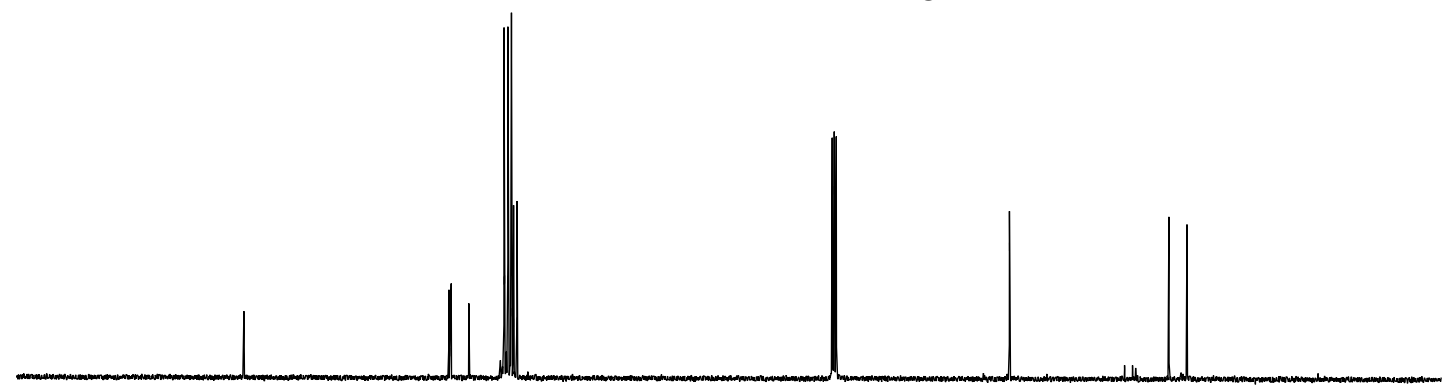

$\begin{array}{rlllllllllll}200 & 190 & 180 & 170 & 160 & 150 & 140 & 130 & 120 & 110 & 100 & 100 \\ \mathrm{f} 1(\mathrm{ppm})\end{array}$

(Z)-N-(1,2-diphenylvinyl)- $N$-(4-methylbenzyl)acetamide (3s)

${ }^{1} \mathrm{H}$ NMR $\left(400 \mathrm{MHz}, \mathrm{CDCl}_{3}\right)$ 


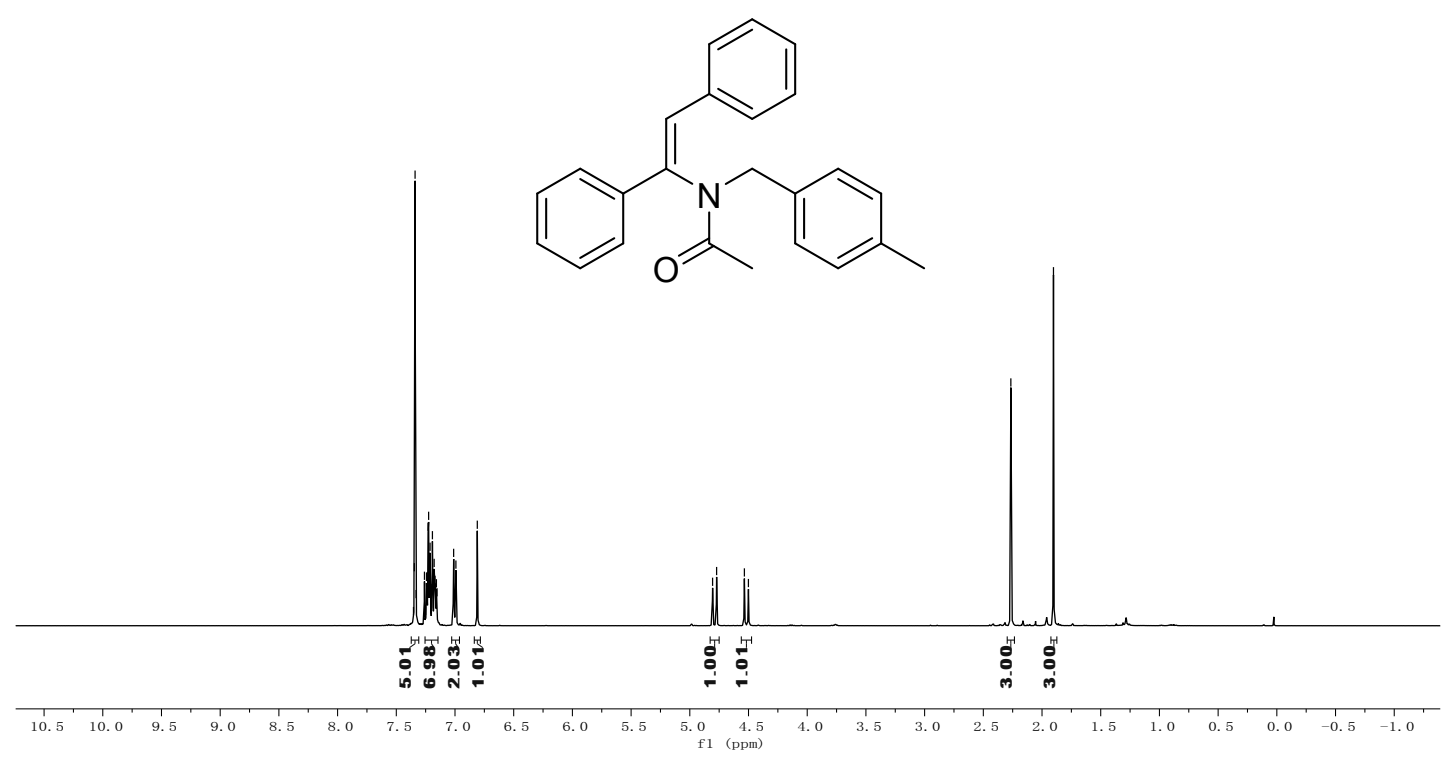

(Z)- $N$-(1,2-diphenylvinyl)- $N$-(4-methylbenzyl)acetamide (3s)

${ }^{13} \mathrm{C} \mathrm{NMR}\left(101 \mathrm{MHz}, \mathrm{CDCl}_{3}\right)$

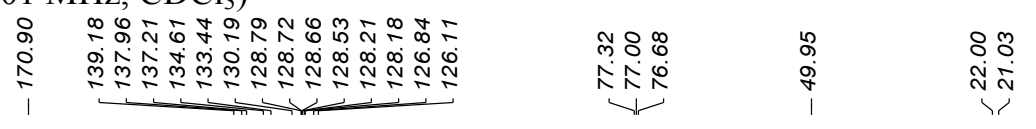
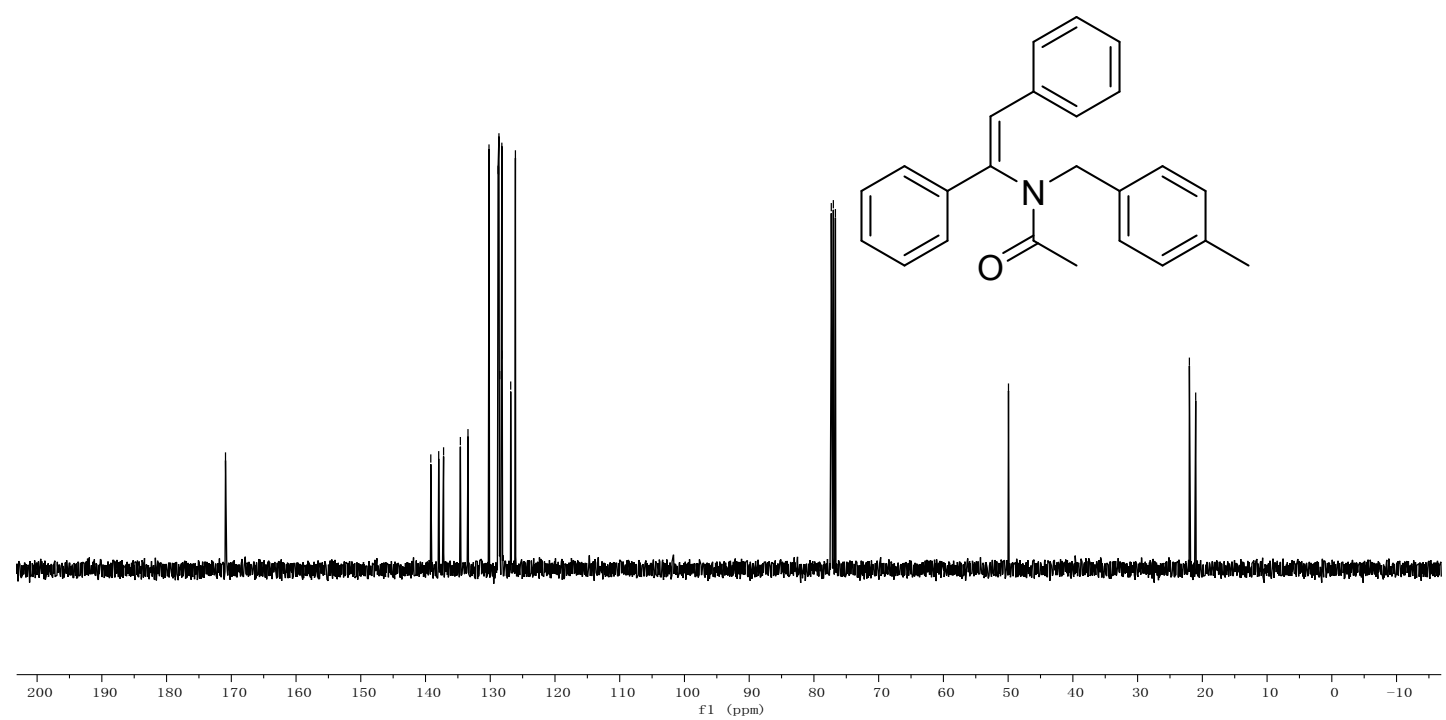

(Z)- $N$-(1,2-diphenylvinyl)- $N$-(4-fluorobenzyl)acetamide (3t)

${ }^{1} \mathrm{H}$ NMR $\left(400 \mathrm{MHz}, \mathrm{CDCl}_{3}\right)$ 
<smiles>CC(=O)N(Cc1ccc(F)cc1)/C(=C\c1ccccc1)c1ccccc1</smiles>

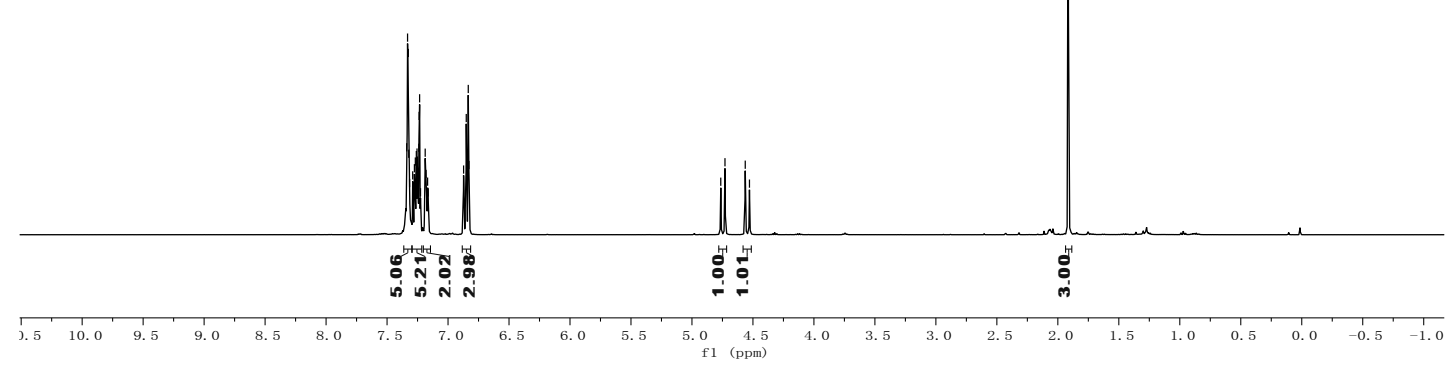

(Z)- $N$-(1,2-diphenylvinyl)- $N$-(4-fluorobenzyl)acetamide (3t) ${ }^{13} \mathrm{C}$ NMR $\left(101 \mathrm{MHz}, \mathrm{CDCl}_{3}\right)$

\%

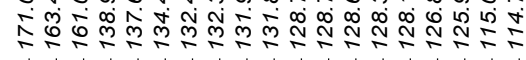

\section{लำ}

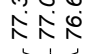

葡

के

$\underset{\substack{\infty \\ i}}{\stackrel{8}{+}}$

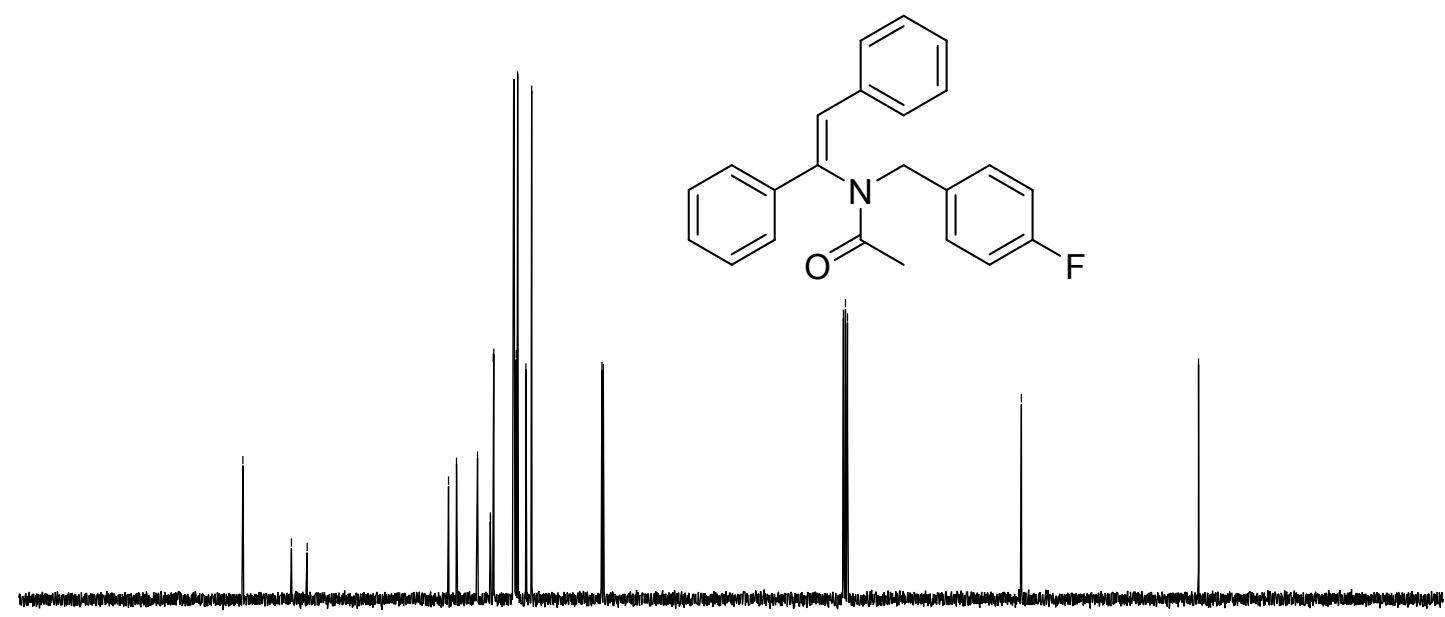

(Z)- $N$-(1,2-diphenylvinyl)- $N$-methylacetamide (3u)

${ }^{1} \mathrm{H}$ NMR $\left(400 \mathrm{MHz}, \mathrm{CDCl}_{3}\right)$ 


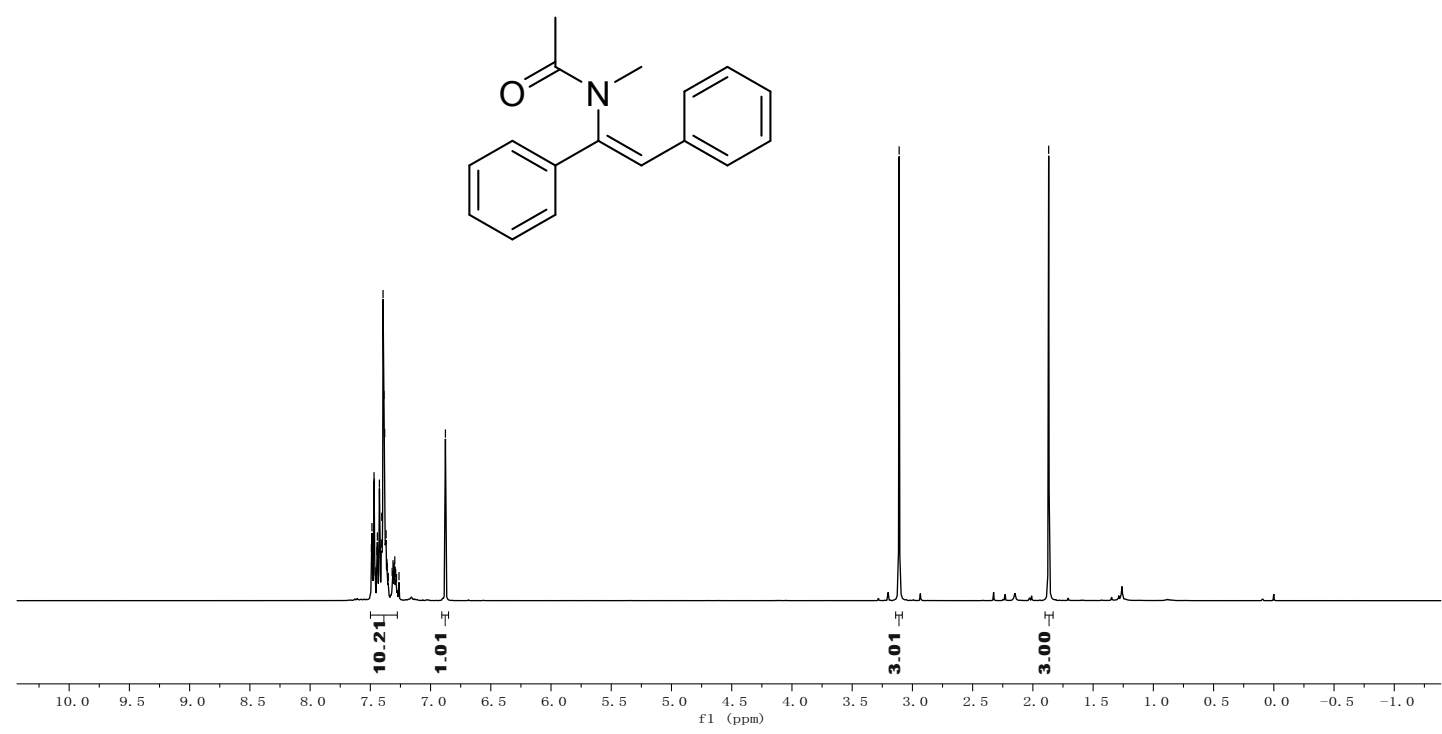

(Z)- $N$-(1,2-diphenylvinyl)- $N$-methylacetamide (3u)

${ }^{13} \mathrm{C}$ NMR $\left(101 \mathrm{MHz}, \mathrm{CDCl}_{3}\right)$

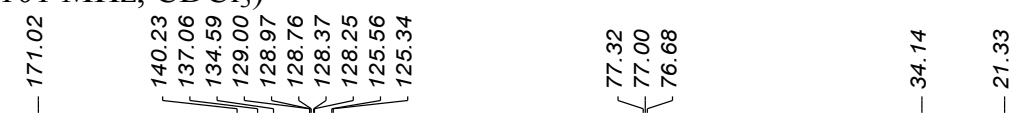
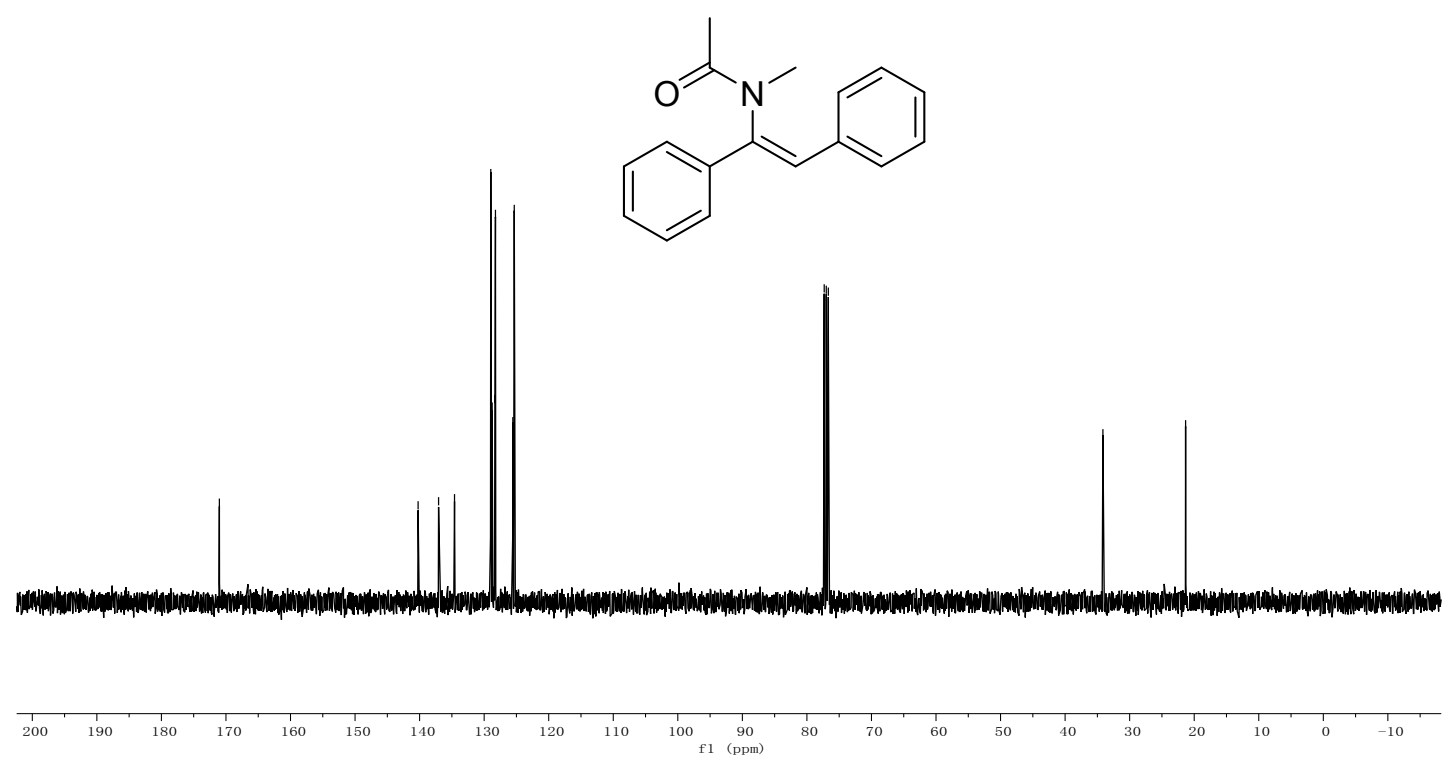

(Z)- $N$-benzyl- $N$-(1,2-diphenylvinyl)propionamide (3v)

${ }^{1} \mathrm{H}$ NMR $\left(400 \mathrm{MHz}, \mathrm{CDCl}_{3}\right)$ 


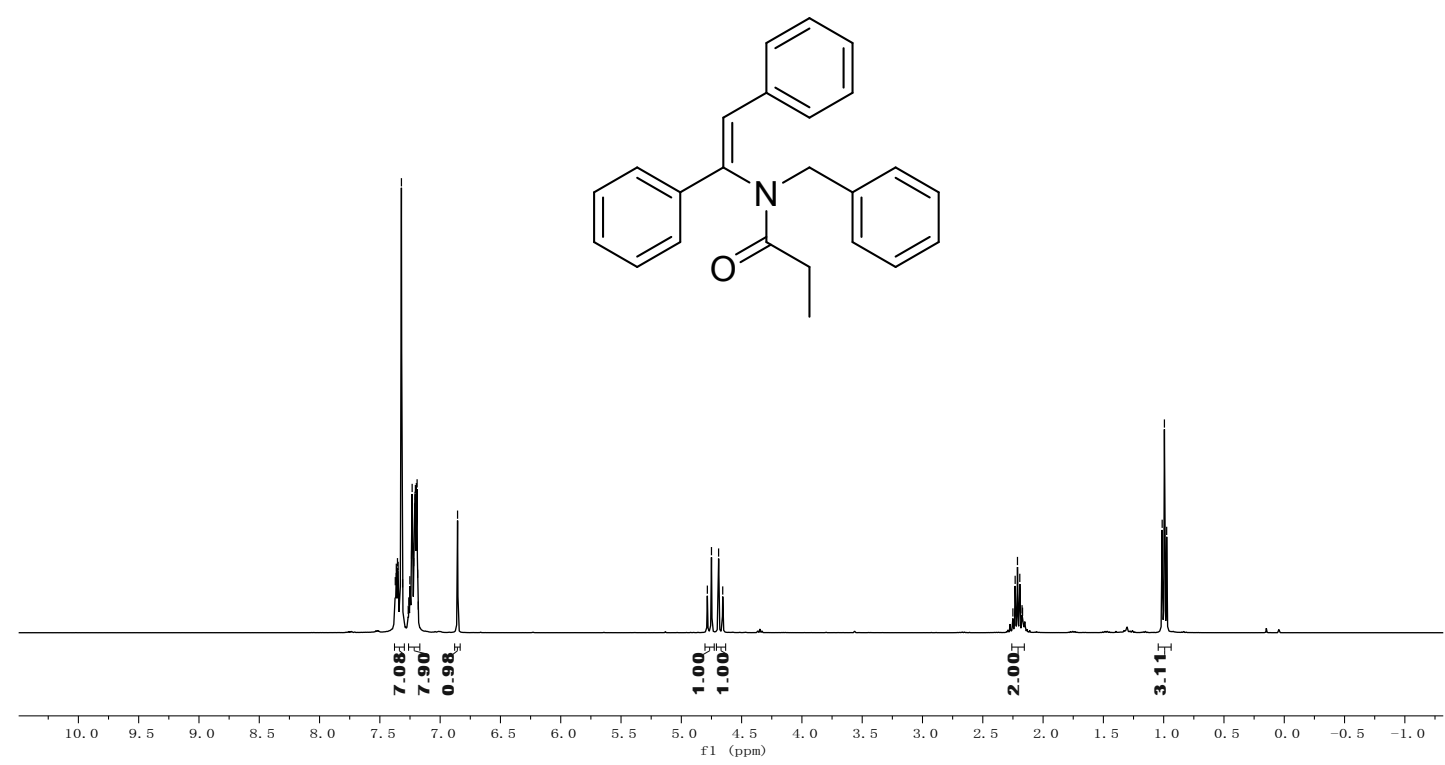

(Z)- $N$-benzyl- $N$-(1,2-diphenylvinyl)propionamide (3v)

${ }^{13} \mathrm{C}$ NMR (101 MHz, $\left.\mathrm{CDCl}_{3}\right)$

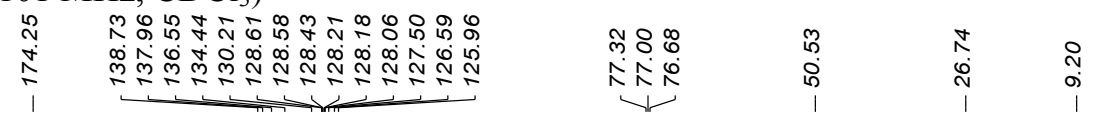
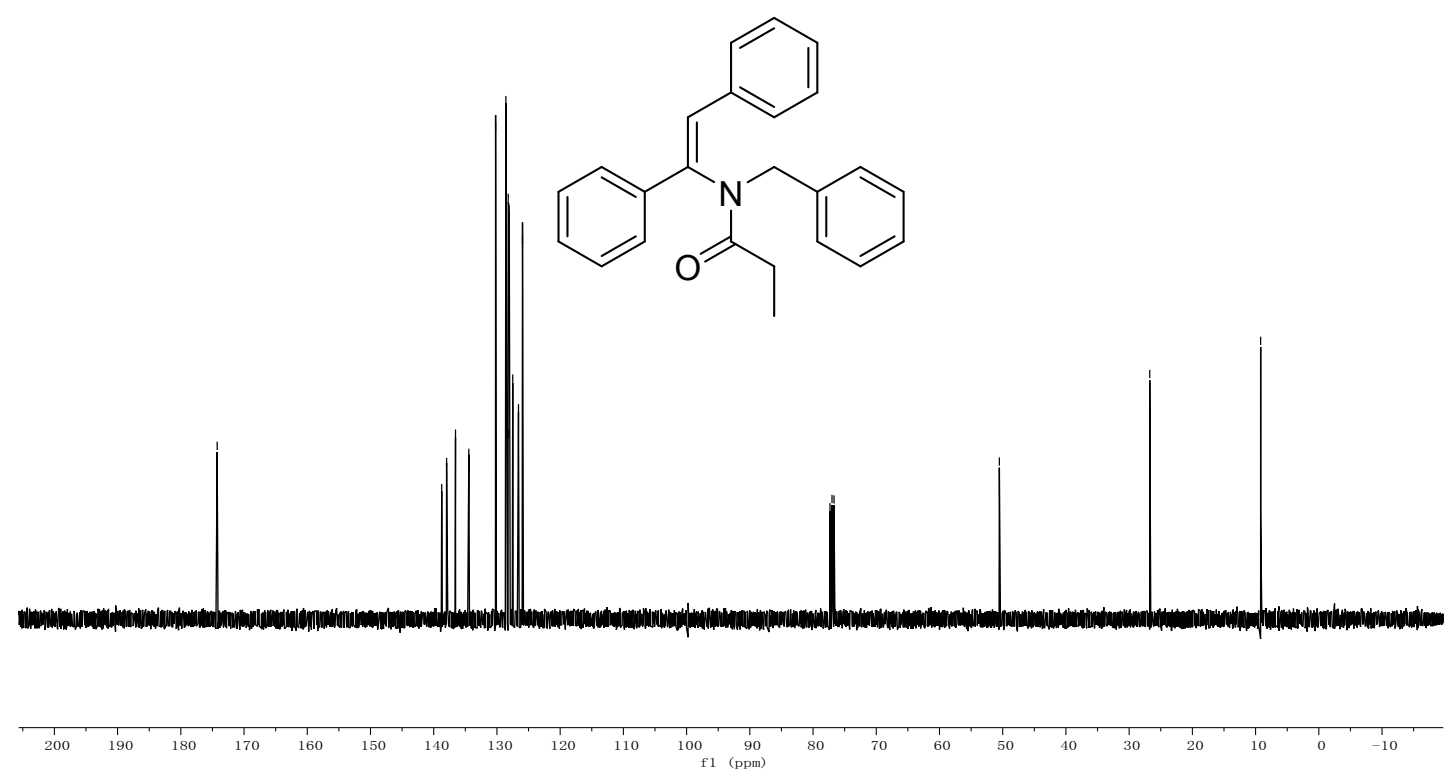

(Z)- $N$-benzyl- $N$-(1-phenyl-2-(p-tolyl)vinyl)acetamide (4b)

${ }^{1} \mathrm{H}$ NMR $\left(400 \mathrm{MHz}, \mathrm{CDCl}_{3}\right)$ 


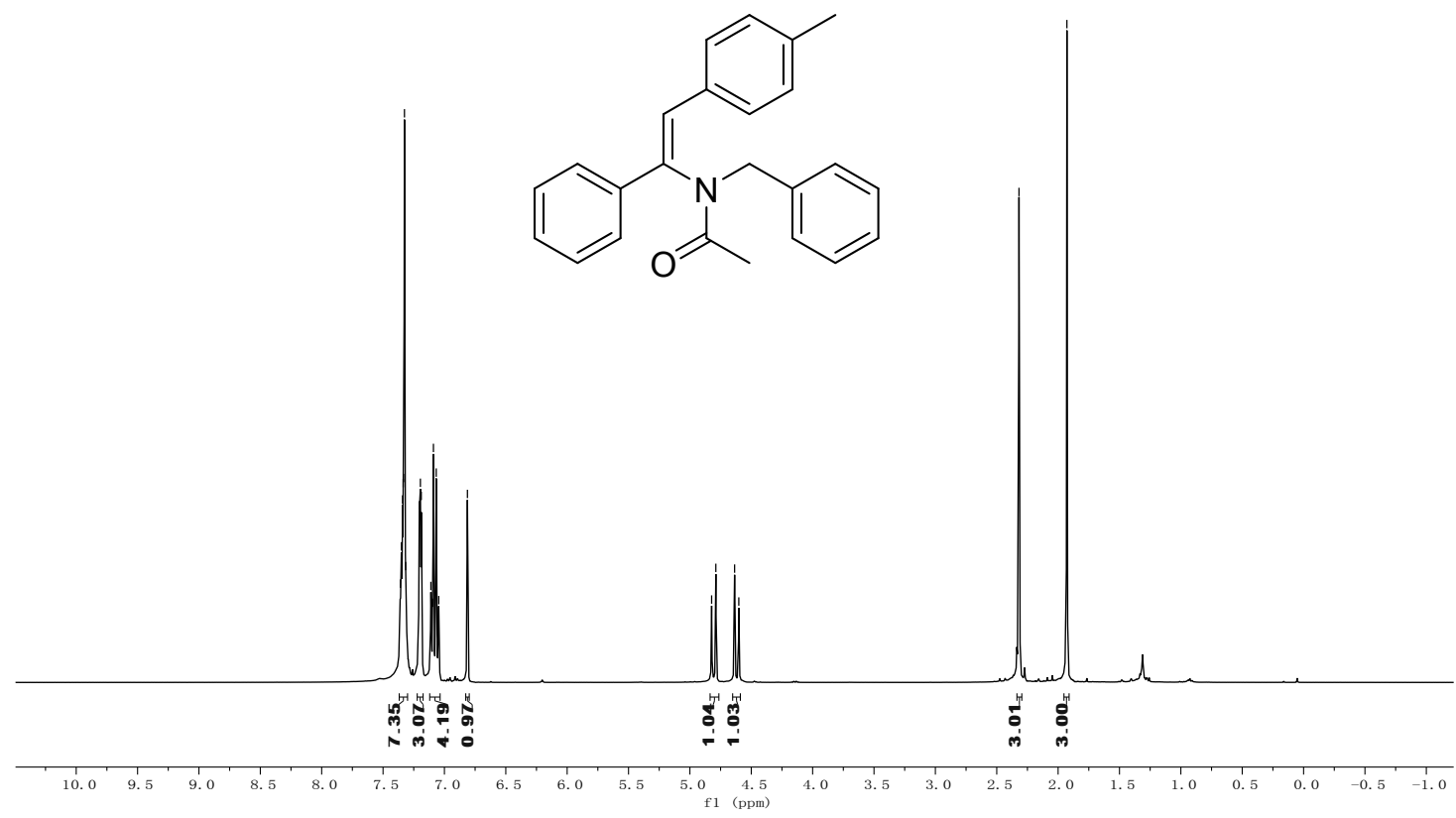

(Z)- $N$-benzyl- $N$-(1-phenyl-2-(p-tolyl)vinyl)acetamide (4b) ${ }^{13} \mathrm{C}$ NMR (101 MHz, $\mathrm{CDCl}_{3}$ )

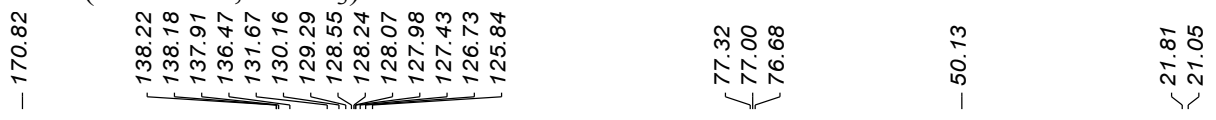

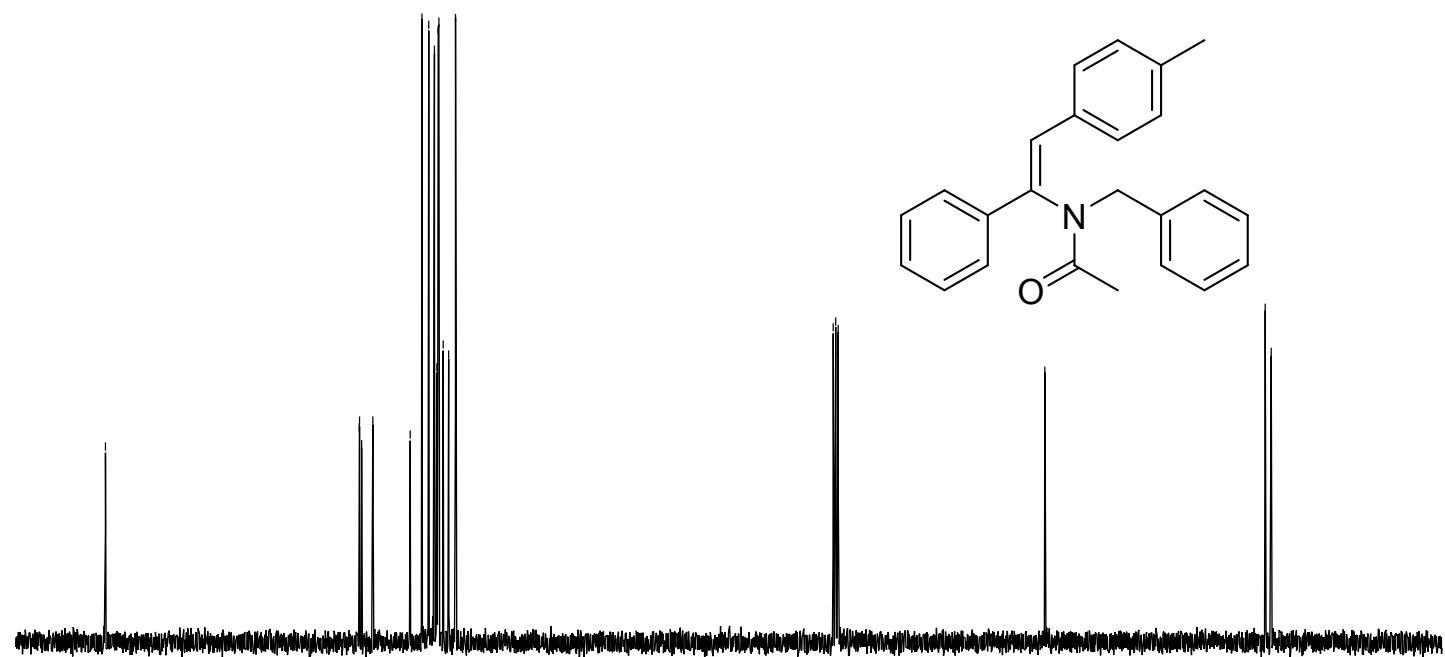

(Z)- $N$-benzyl- $N$-(1-phenyl-2-(m-tolyl)vinyl)acetamide (4c) ${ }^{1} \mathrm{H}$ NMR $\left(400 \mathrm{MHz}, \mathrm{CDCl}_{3}\right)$ 


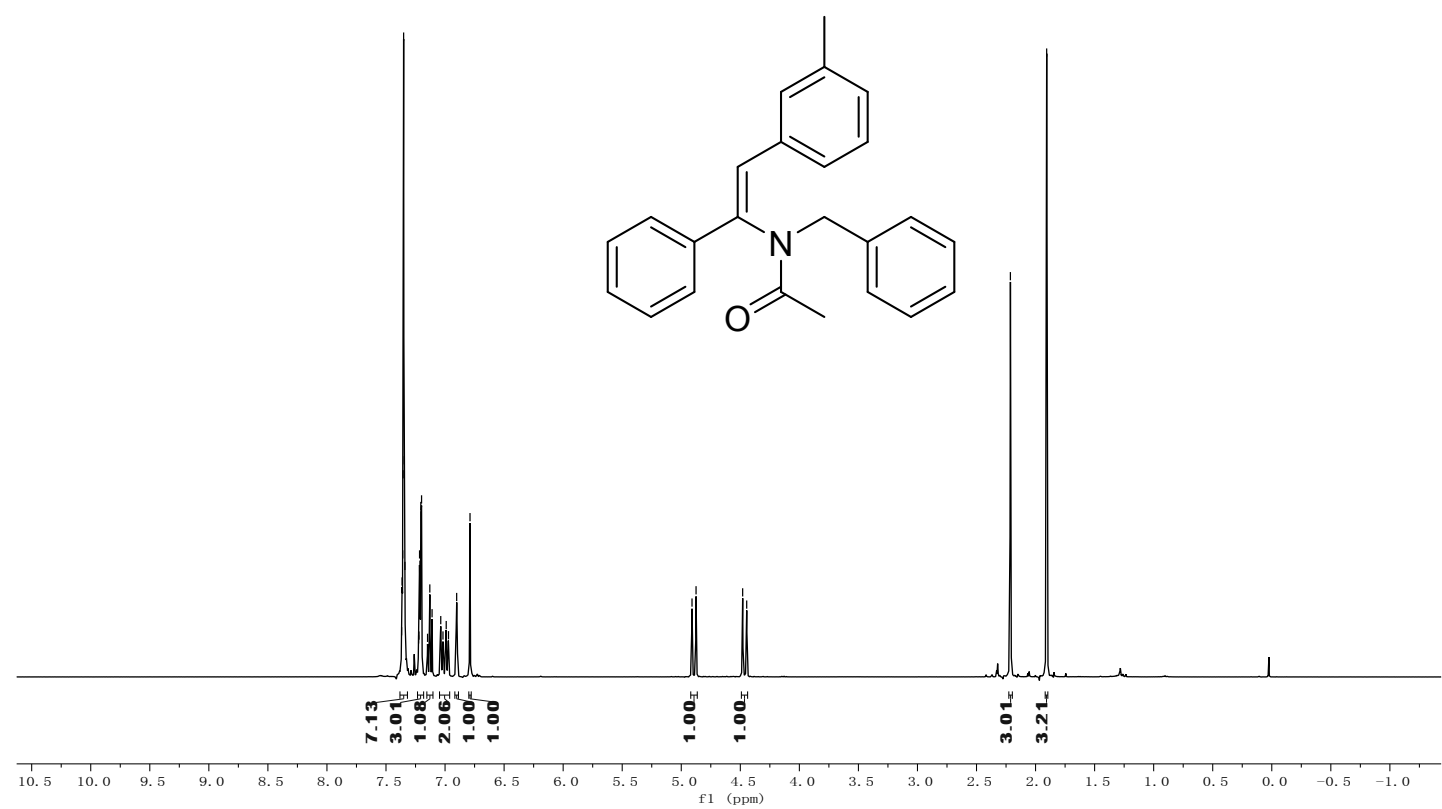

(Z)- $N$-benzyl- $N$-(1-phenyl-2-(m-tolyl)vinyl)acetamide (4c) ${ }^{13} \mathrm{C}$ NMR (101 MHz, $\mathrm{CDCl}_{3}$ )
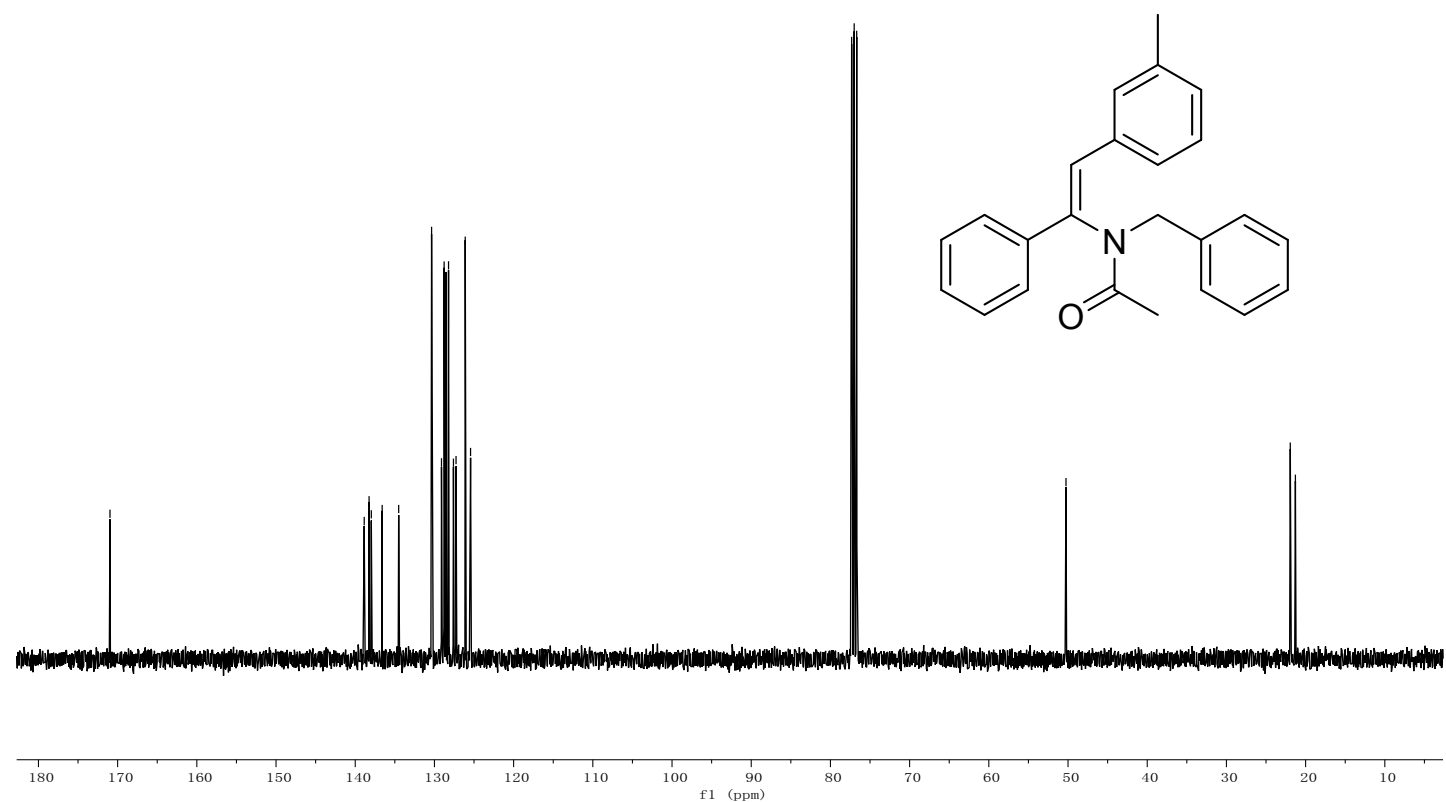

(Z)- $N$-benzyl- $N$-(1-phenyl-2-(o-tolyl)vinyl)acetamide (4d)

${ }^{1} \mathrm{H}$ NMR $\left(400 \mathrm{MHz}, \mathrm{CDCl}_{3}\right)$ 


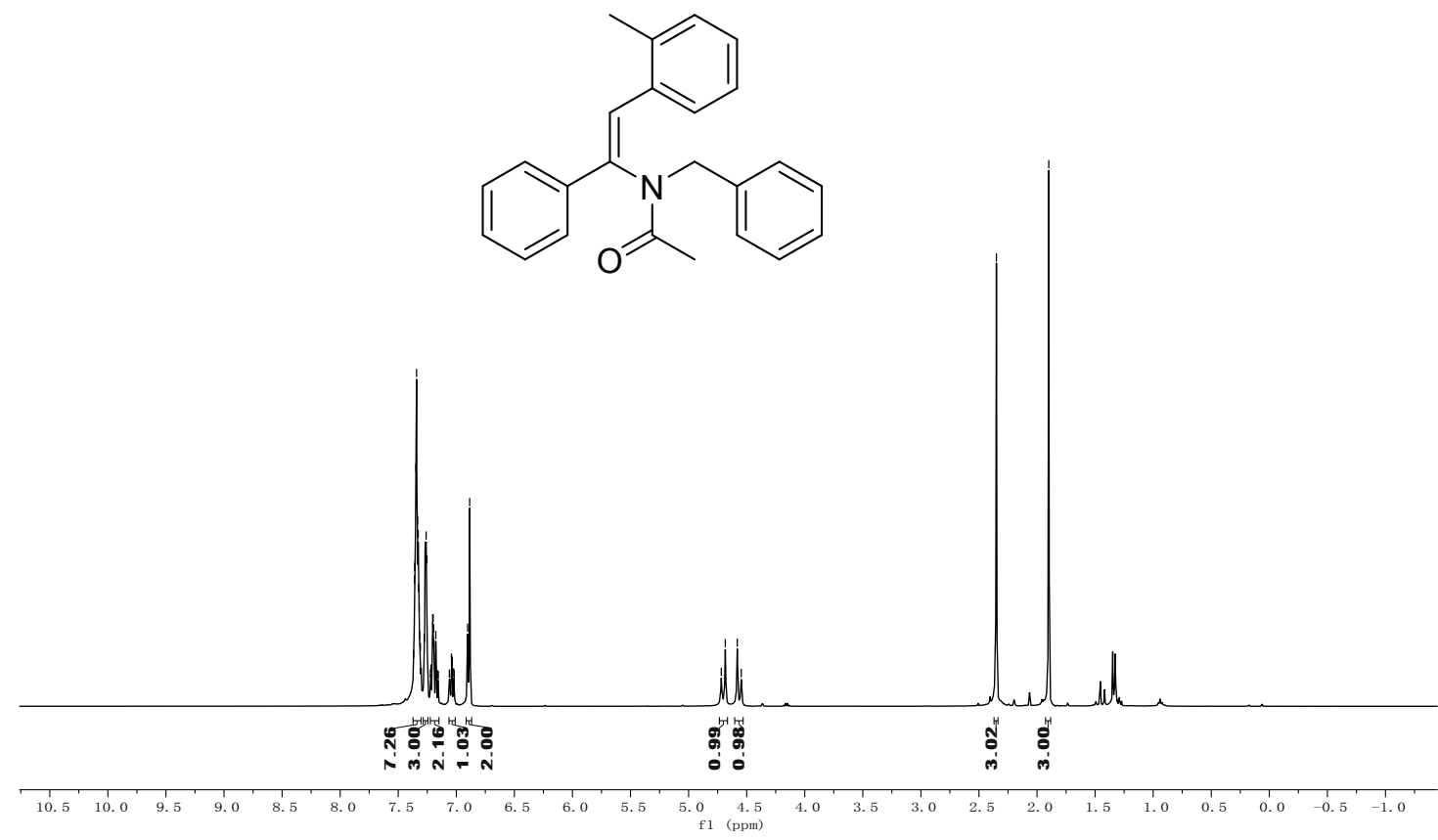

(Z)- $N$-benzyl- $N$-(1-phenyl-2-(o-tolyl)vinyl)acetamide (4d)

${ }^{13} \mathrm{C}$ NMR $\left(101 \mathrm{MHz}, \mathrm{CDCl}_{3}\right)$

+ क क

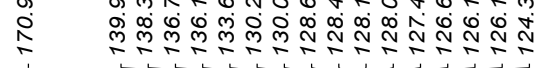

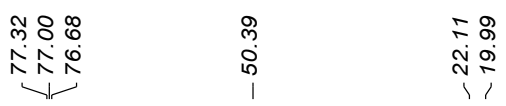

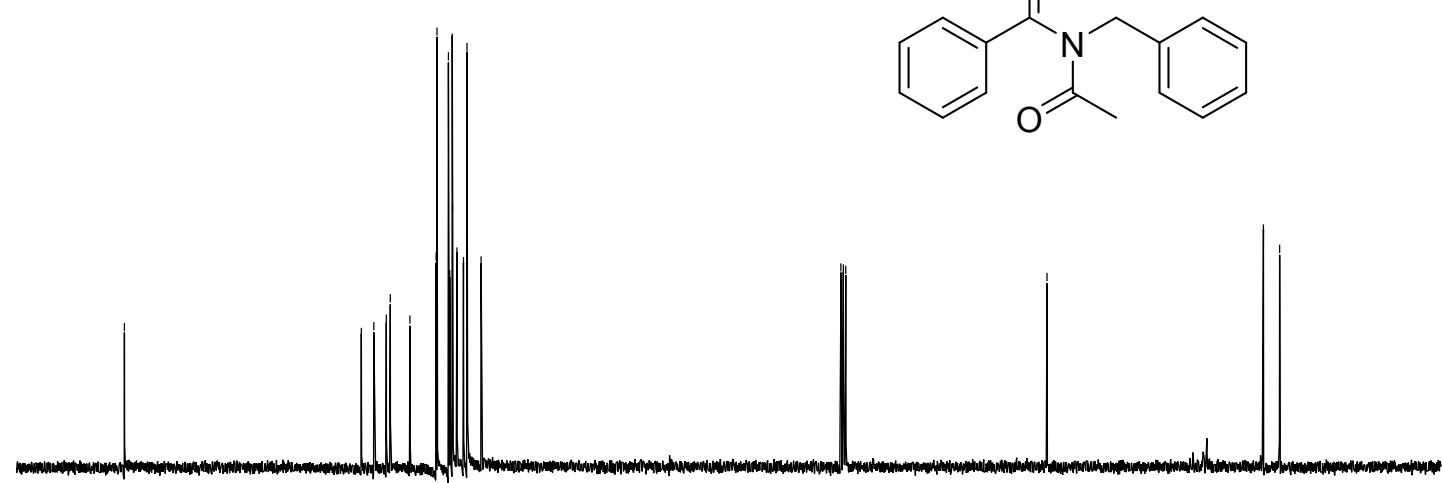

(Z)- $N$-benzyl- $N$-(2-(3,5-dimethylphenyl)-1-phenylvinyl)acetamide (4e) ${ }^{1} \mathrm{H}$ NMR $\left(400 \mathrm{MHz}, \mathrm{CDCl}_{3}\right)$ 


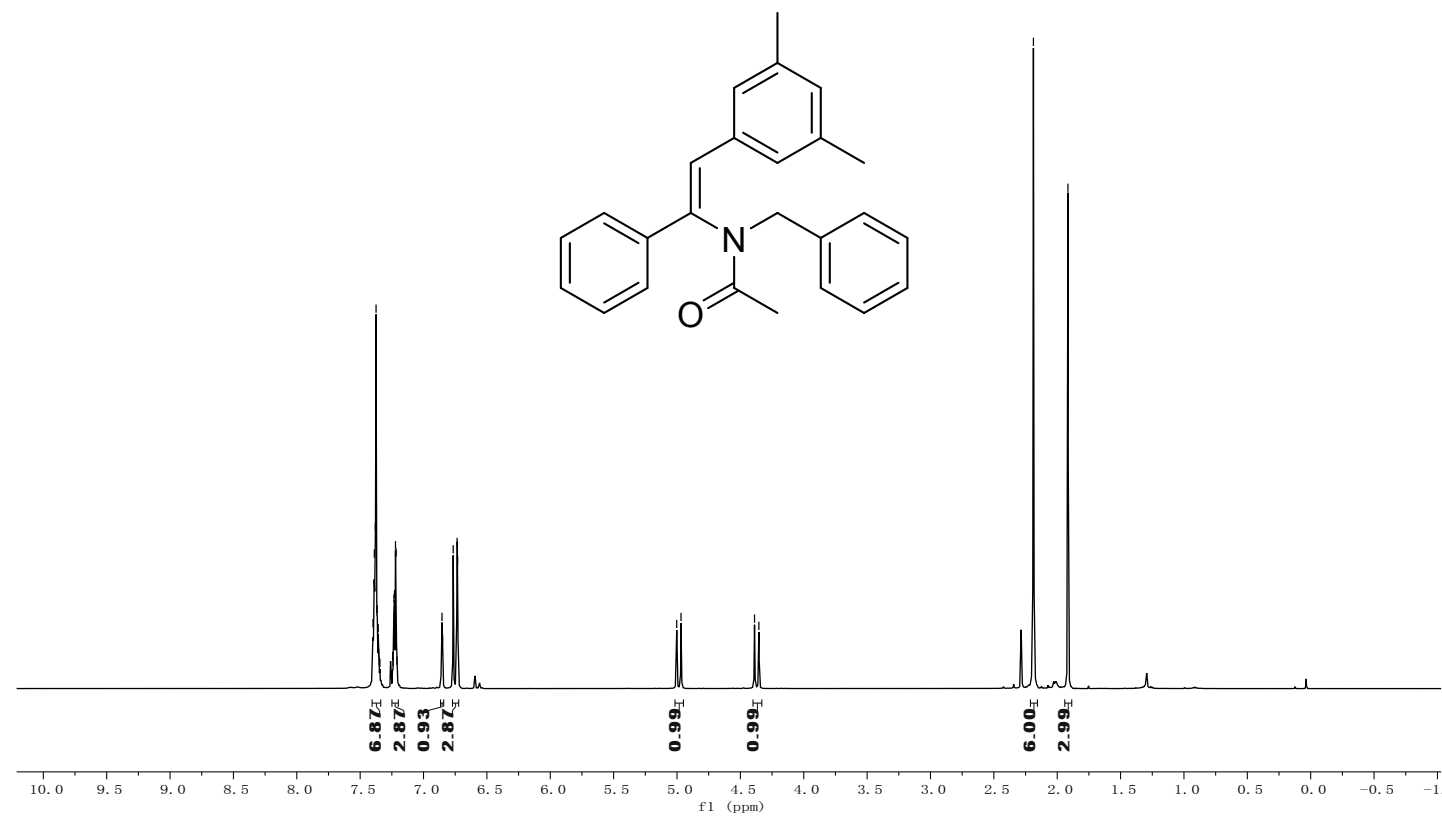

(Z)- $N$-benzyl- $N$-(2-(3,5-dimethylphenyl)-1-phenylvinyl)acetamide (4e) ${ }^{13} \mathrm{C}$ NMR (101 MHz, $\left.\mathrm{CDCl}_{3}\right)$

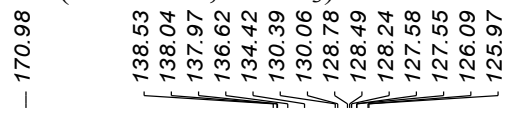

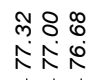

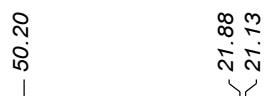

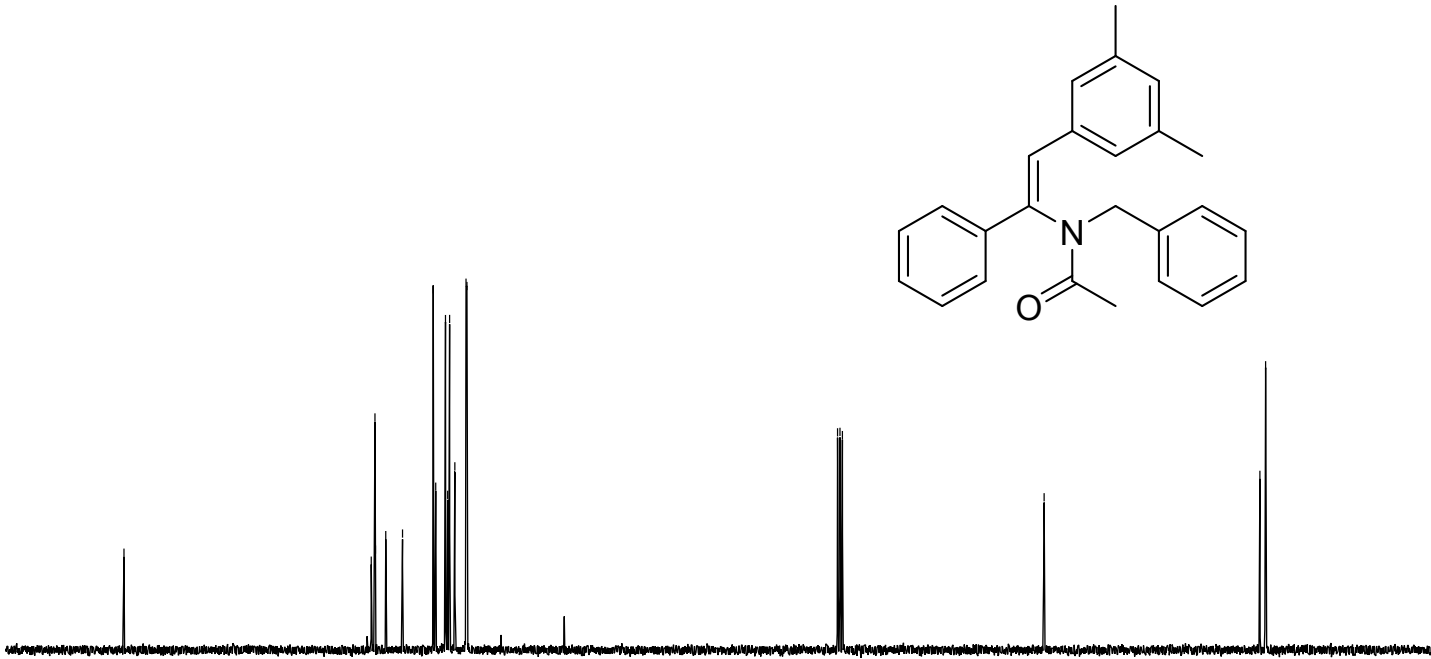

(Z)- $N$-benzyl- $N$-(2-(4-(tert-butyl)phenyl)-1-phenylvinyl)acetamide (4f) ${ }^{1} \mathrm{H}$ NMR $\left(400 \mathrm{MHz}, \mathrm{CDCl}_{3}\right)$ 
<smiles>CC(=O)N(Cc1ccccc1)/C(=C\c1ccc(C(C)(C)C)cc1)c1ccccc1</smiles>

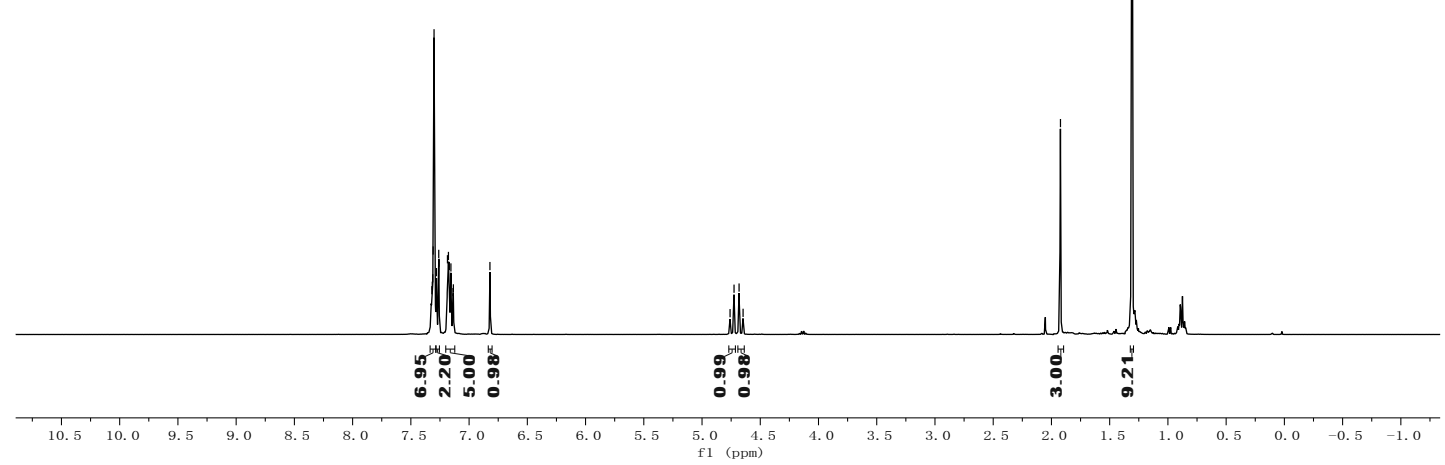

(Z)- $N$-benzyl- $N$-(2-(4-(tert-butyl)phenyl)-1-phenylvinyl)acetamide (4f) ${ }^{13} \mathrm{C}$ NMR $\left(101 \mathrm{MHz}, \mathrm{CDCl}_{3}\right)$

$\begin{array}{ll}1 & 1 \\ 0 & 0\end{array}$

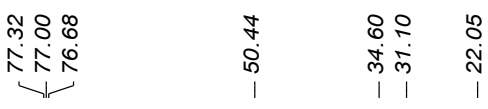
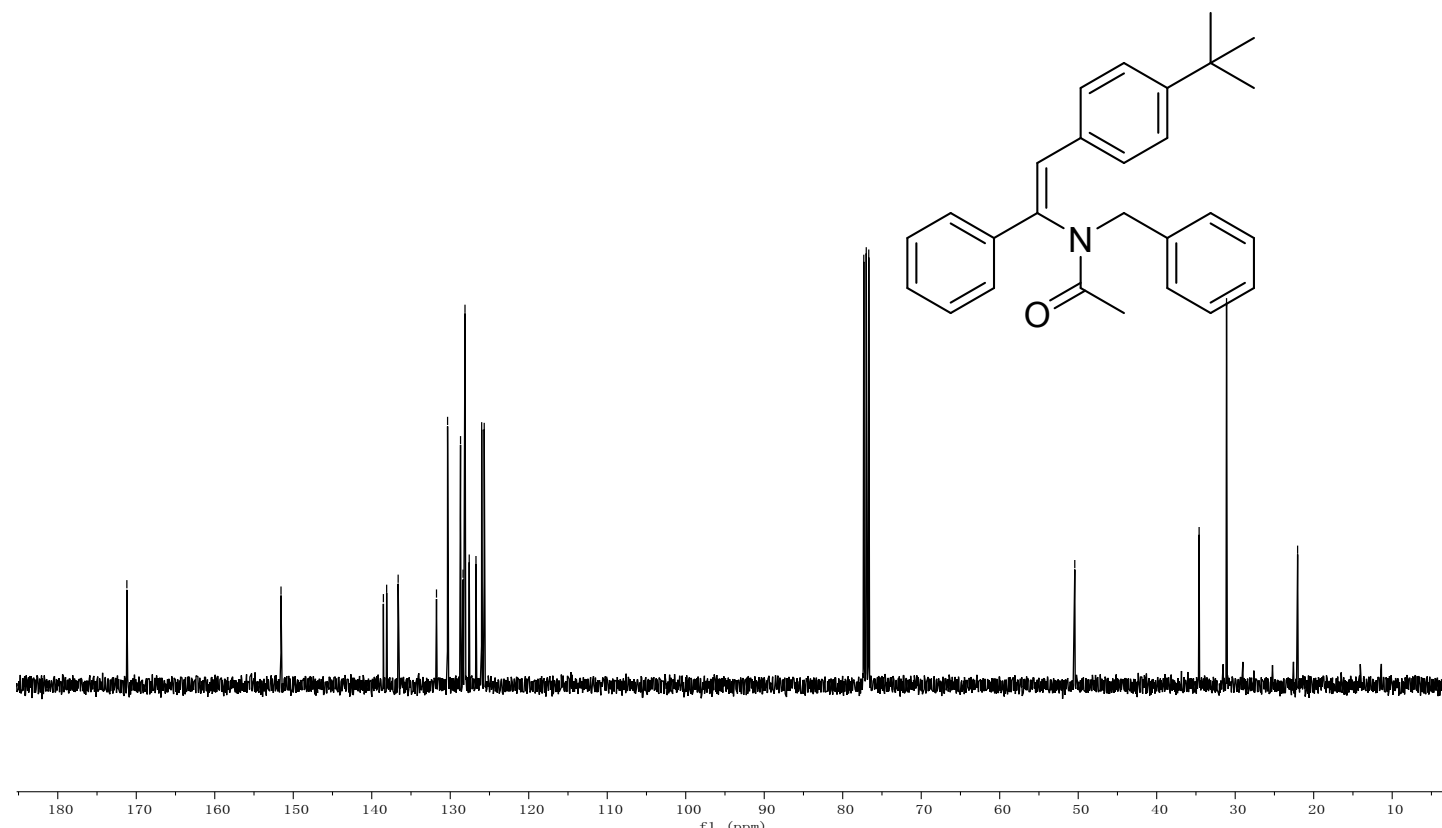

(Z)- $N$-benzyl- $N$-(2-(3-chloro-4-methylphenyl)-1-phenylvinyl)acetamide (4g) ${ }^{1} \mathrm{H}$ NMR $\left(400 \mathrm{MHz}, \mathrm{CDCl}_{3}\right)$ 


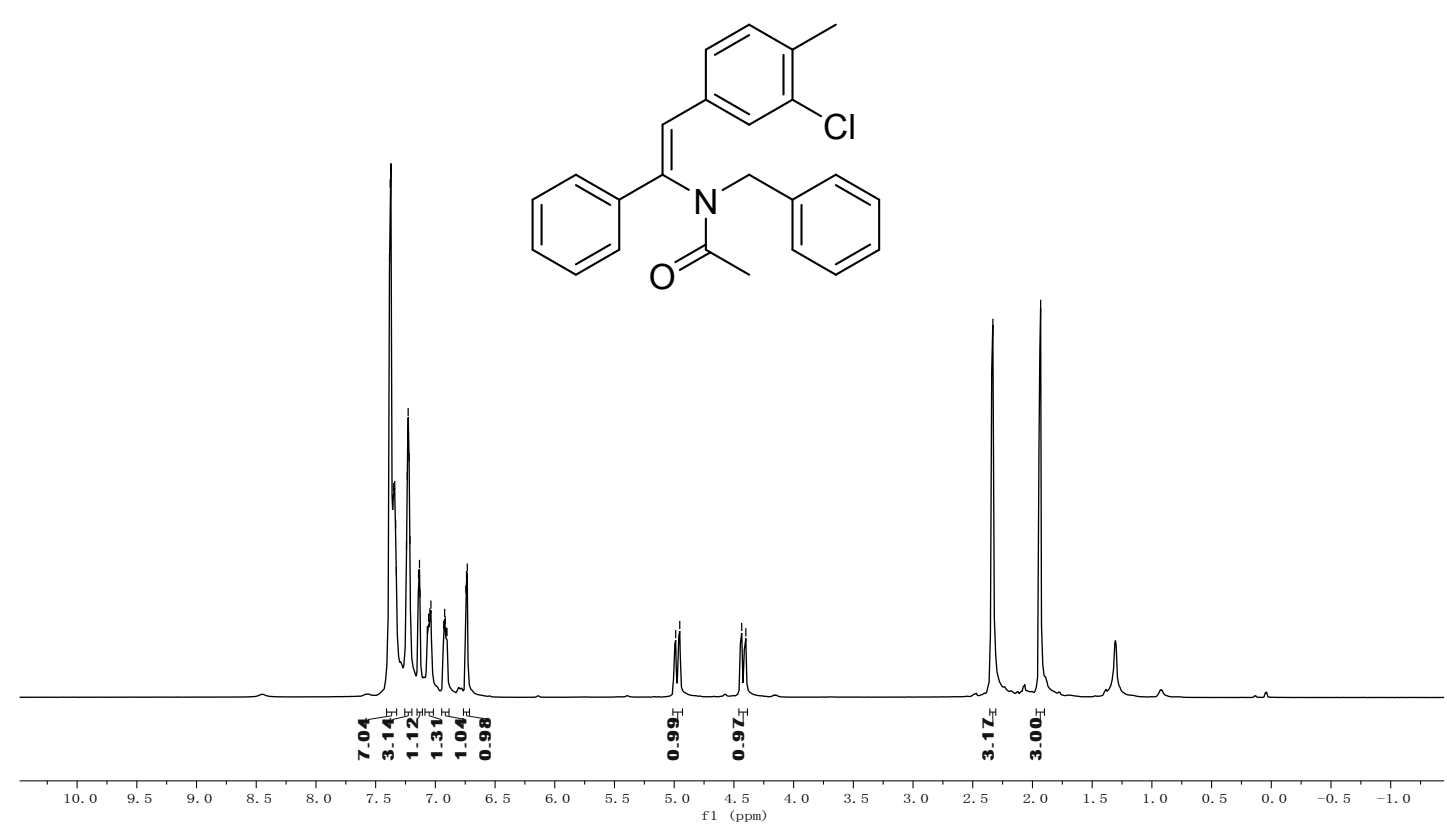

(Z)-N-benzyl- $N$-(2-(3-chloro-4-methylphenyl)-1-phenylvinyl)acetamide (4g) ${ }^{13} \mathrm{C}$ NMR (101 MHz, $\left.\mathrm{CDCl}_{3}\right)$

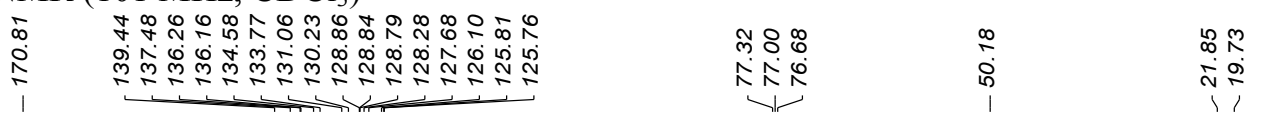

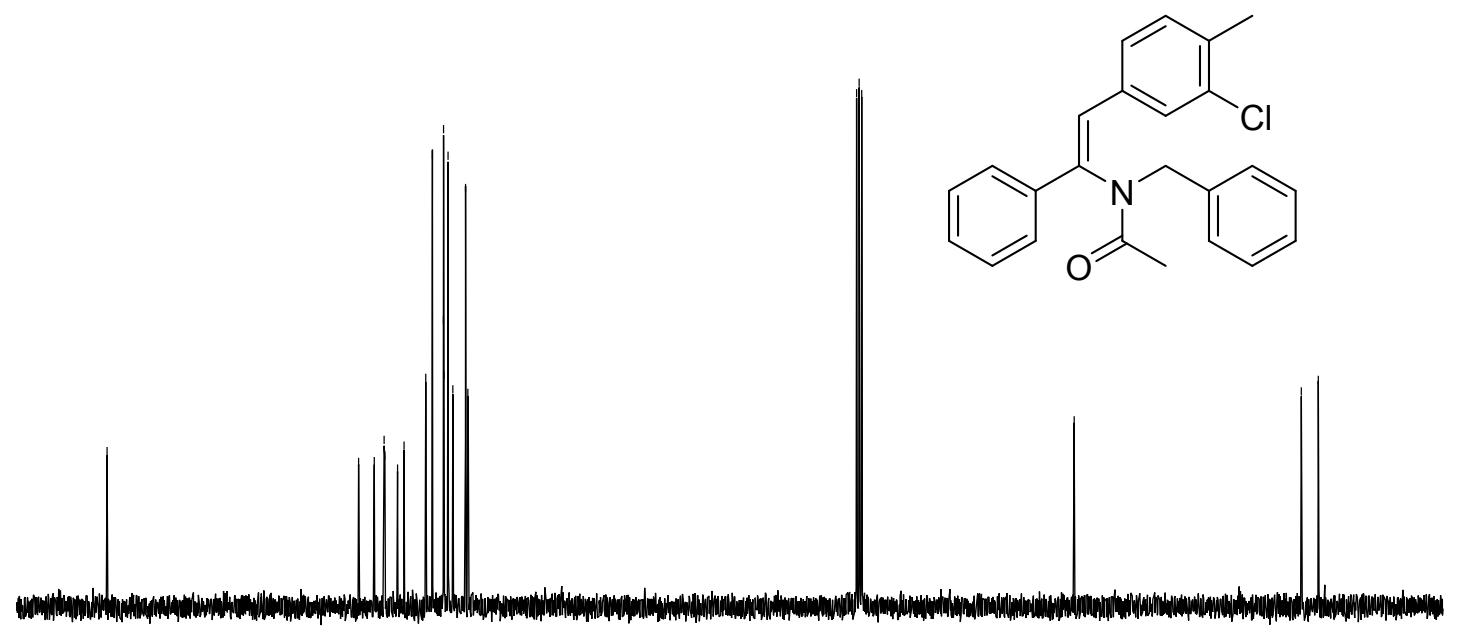

(Z)- $N$-benzyl- $N$-(2-(4-methoxyphenyl)-1-phenylvinyl)acetamide (4h) ${ }^{1} \mathrm{H}$ NMR $\left(400 \mathrm{MHz}, \mathrm{CDCl}_{3}\right)$ 
<smiles>COc1ccc(/C=C(/c2ccccc2)N(Cc2ccccc2)C(C)=O)cc1</smiles>

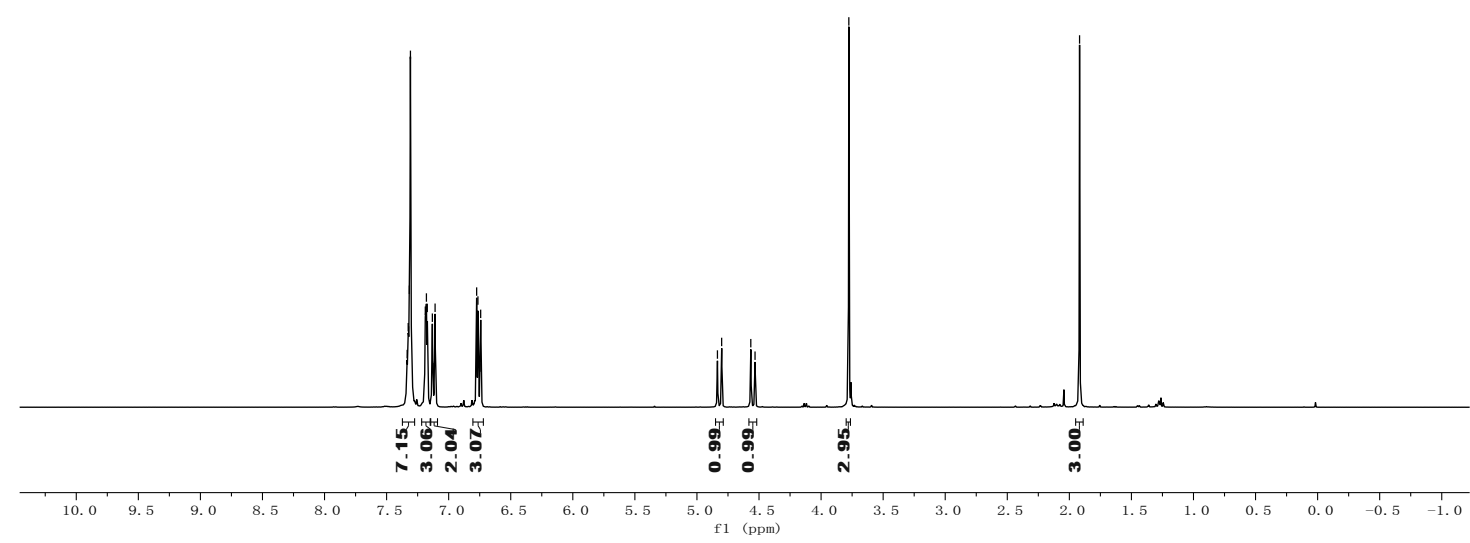

(Z)- $N$-benzyl- $N$-(2-(4-methoxyphenyl)-1-phenylvinyl)acetamide (4h) ${ }^{13} \mathrm{C}$ NMR (101 MHz, $\left.\mathrm{CDCl}_{3}\right)$

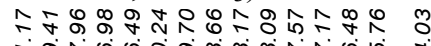

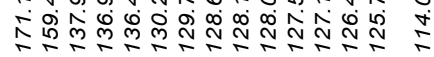
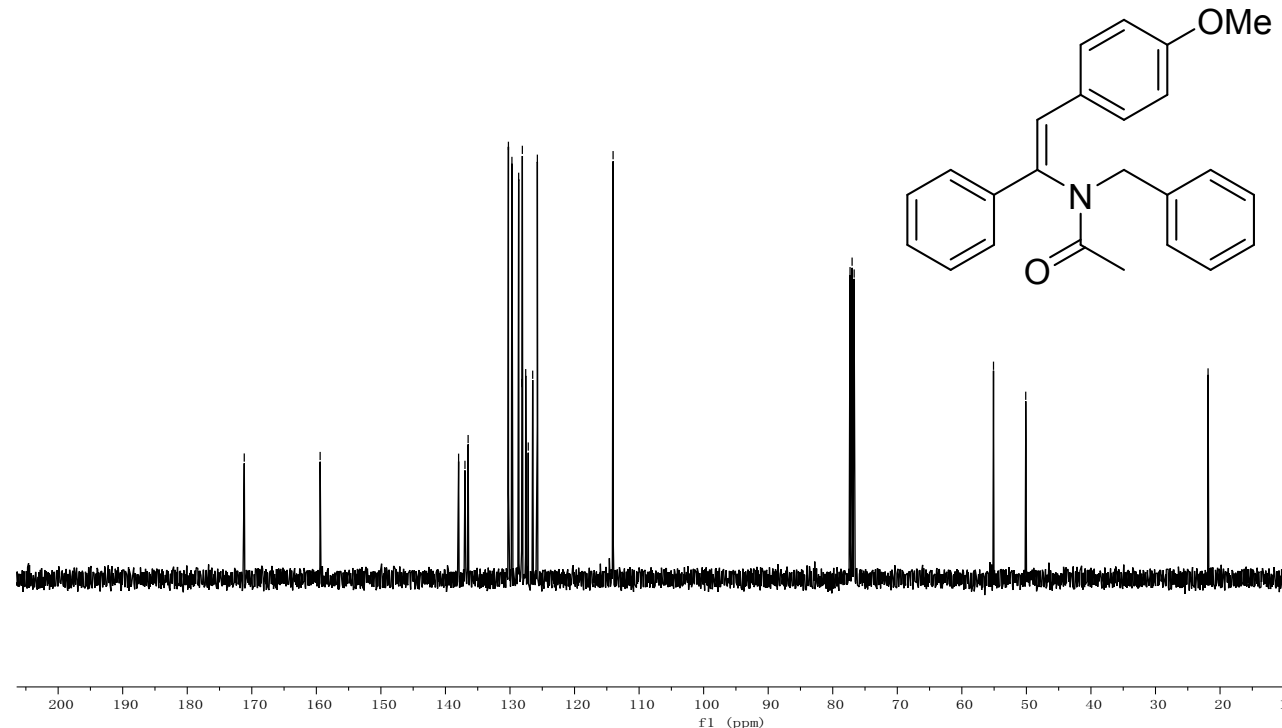

(Z)- $N$-benzyl- $N$-(2-(3-methoxyphenyl)-1-phenylvinyl)acetamide (4i) ${ }^{1} \mathrm{H}$ NMR $\left(400 \mathrm{MHz}, \mathrm{CDCl}_{3}\right)$ 


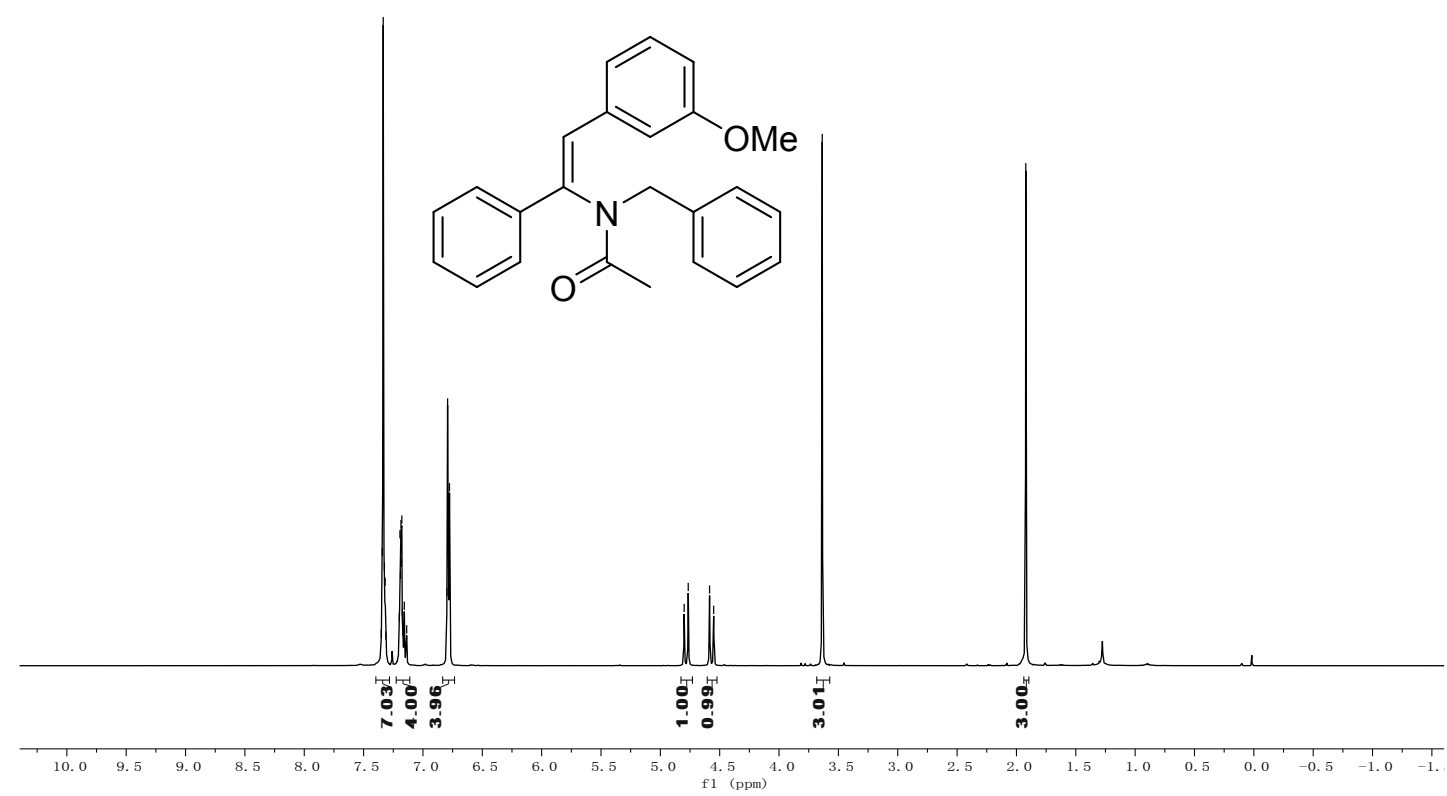

(Z)- $N$-benzyl- $N$-(2-(3-methoxyphenyl)-1-phenylvinyl)acetamide (4i)

${ }^{13} \mathrm{C}$ NMR $\left(101 \mathrm{MHz}, \mathrm{CDCl}_{3}\right)$

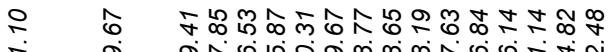
i.

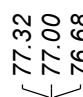
$\stackrel{1}{i}$
究
$\stackrel{\substack{\mathrm{N} \\ 1}}{1}$

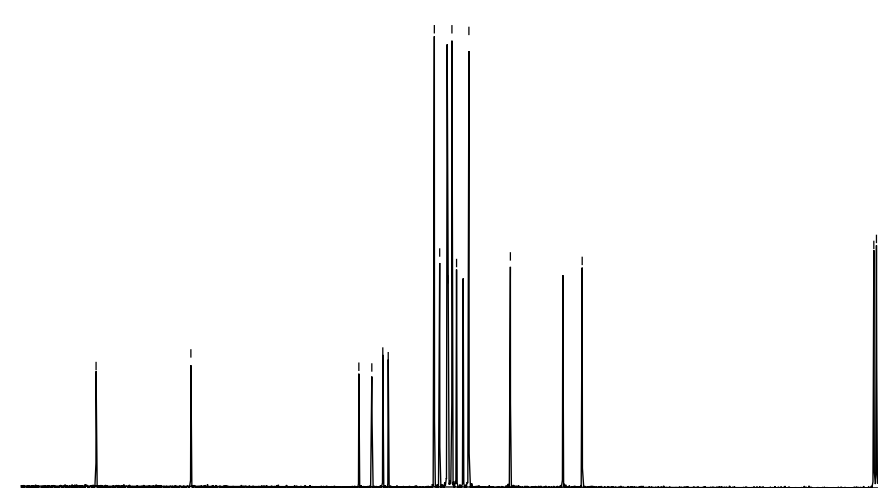<smiles>COc1cccc(/C=C(/c2ccccc2)N(Cc2ccccc2)C(C)=O)c1</smiles>

\begin{tabular}{llllllllll}
\hline 80 & 170 & 160 & 150 & 140 & 130 & 120 & 110 & 100 & $\substack{90 \\
\mathrm{f} 1}$ \\
$(\mathrm{ppm})$ & 1
\end{tabular}

(Z)- $N$-(2-(benzo[d][1,3]dioxol-5-yl)-1-phenylvinyl)- $N$-benzylacetamide (4j)

${ }^{1} \mathrm{H}$ NMR (400 MHz, $\left.\mathrm{CDCl}_{3}\right)$ 


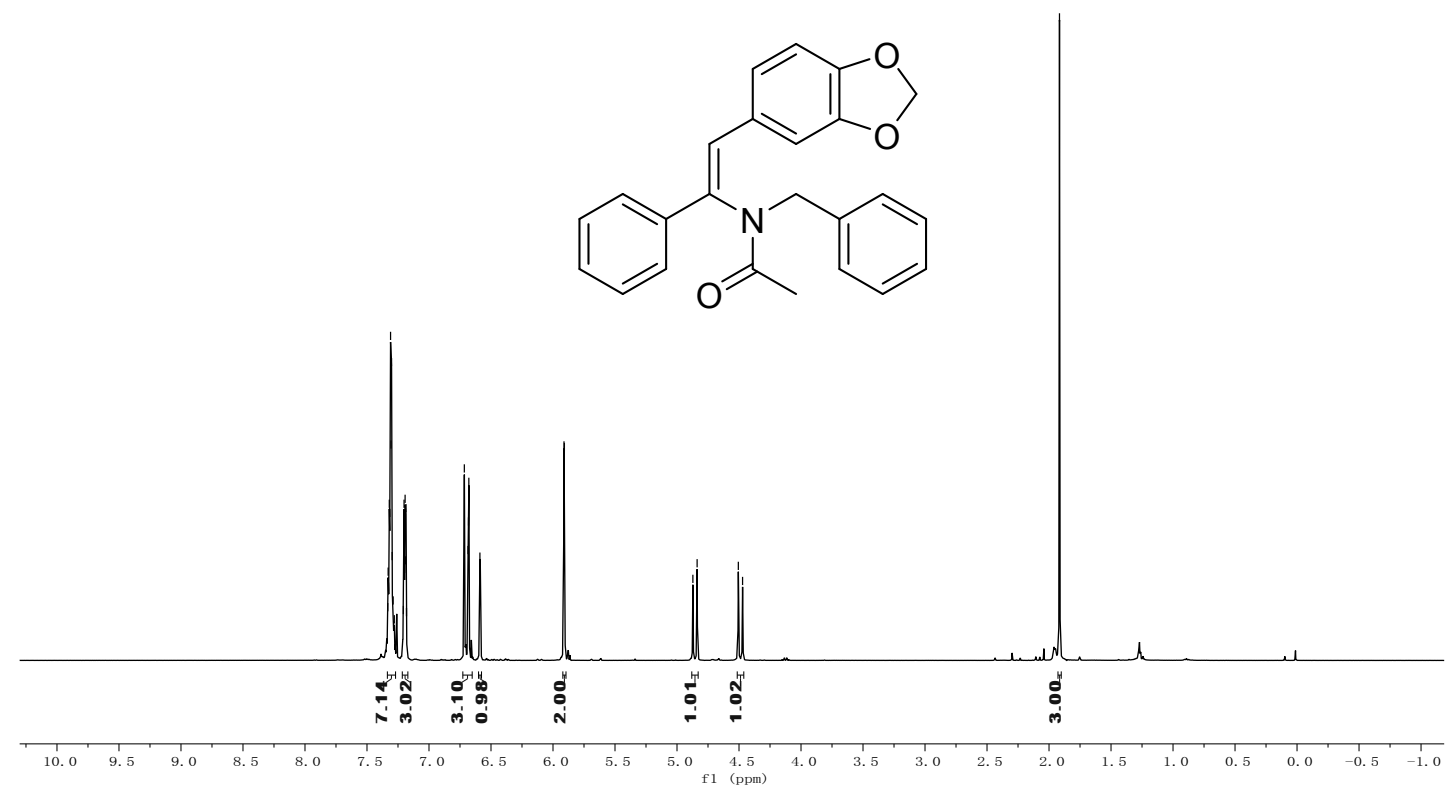

(Z)- $N$-(2-(benzo[d][1,3]dioxol-5-yl)-1-phenylvinyl)- $N$-benzylacetamide (4j) ${ }^{13} \mathrm{C}$ NMR $\left(101 \mathrm{MHz}, \mathrm{CDCl}_{3}\right)$

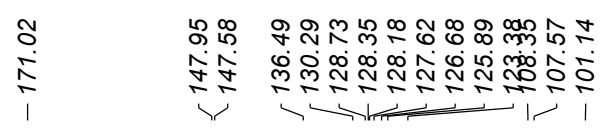

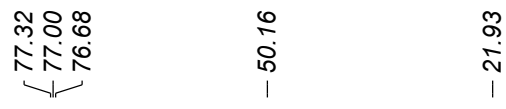
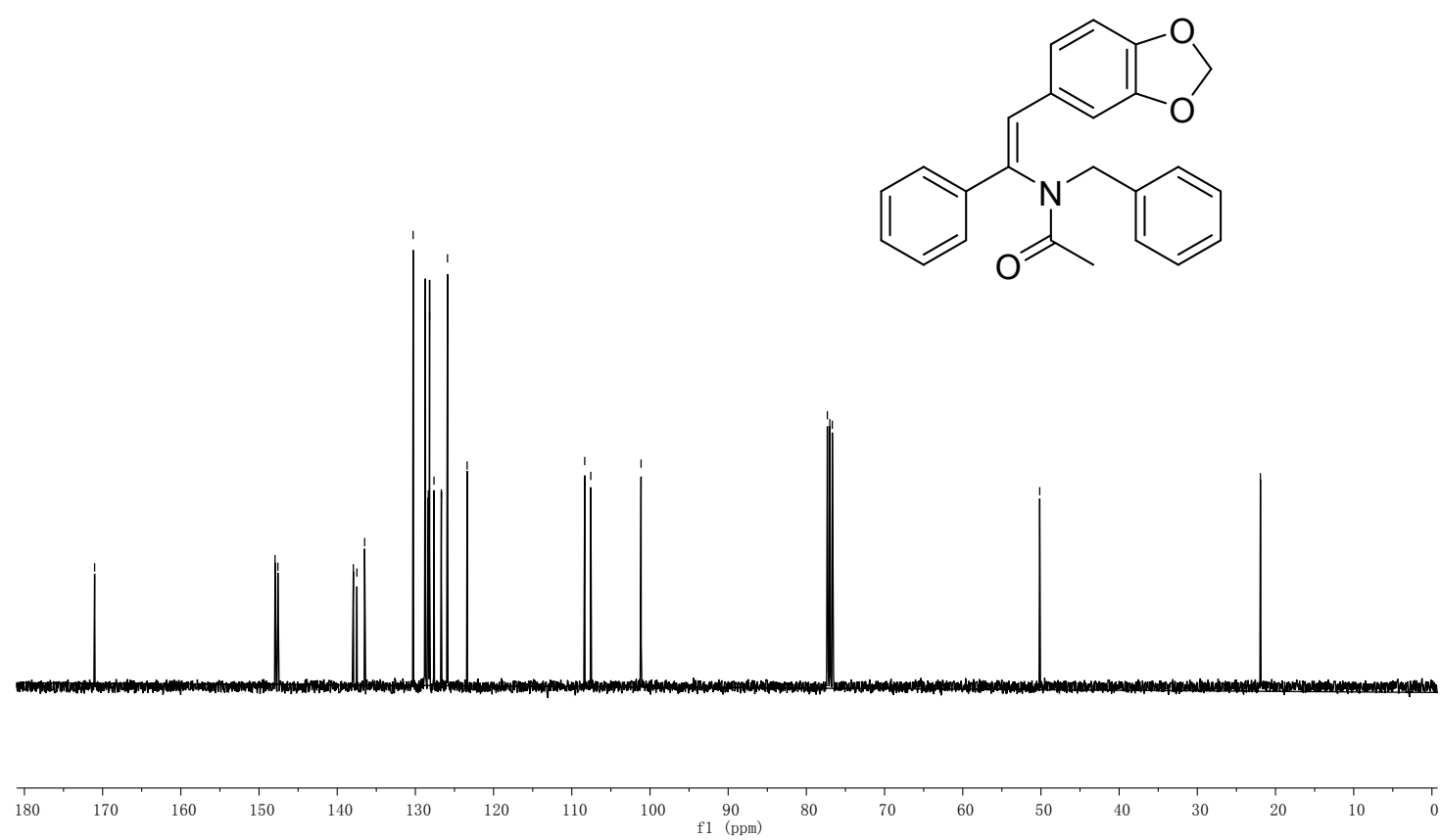

(Z)- $N$-benzyl- $N$-(2-(4-fluorophenyl)-1-phenylvinyl)acetamide (4k) ${ }^{1} \mathrm{H}$ NMR $\left(400 \mathrm{MHz}, \mathrm{CDCl}_{3}\right)$ 
<smiles>CC(=O)N(Cc1ccccc1)/C(=C\c1ccc(F)cc1)c1ccccc1</smiles>

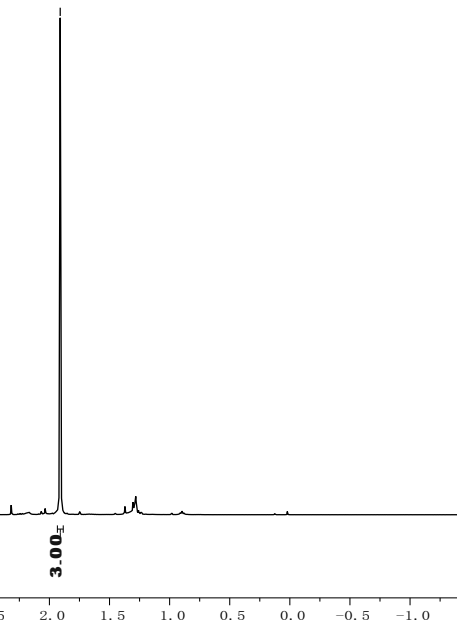

(Z)- $N$-benzyl- $N$-(2-(4-fluorophenyl)-1-phenylvinyl)acetamide (4k)

${ }^{13} \mathrm{C}$ NMR (101 MHz, $\left.\mathrm{CDCl}_{3}\right)$

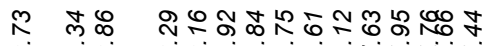

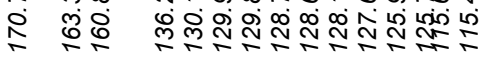

11 ,

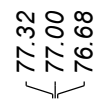

$\begin{array}{ll}5 & \frac{1}{\pi} \\ 0 & 1\end{array}$

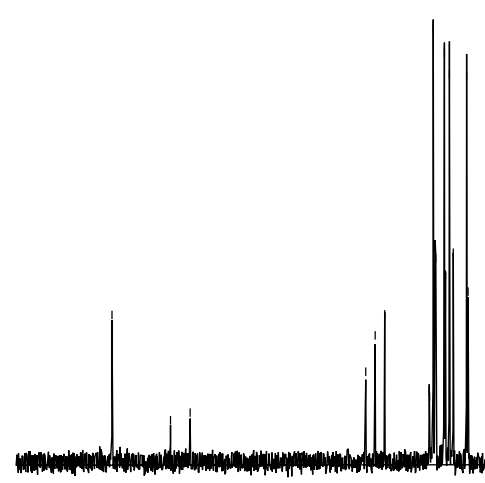<smiles>CC(=O)N(Cc1ccccc1)/C(=C\c1ccc(F)cc1)c1ccccc1</smiles>

180

(Z)- $N$-benzyl- $N$-(2-(4-chlorophenyl)-1-phenylvinyl)acetamide (4I)

${ }^{1} \mathrm{H}$ NMR (400 MHz, $\left.\mathrm{CDCl}_{3}\right)$ 


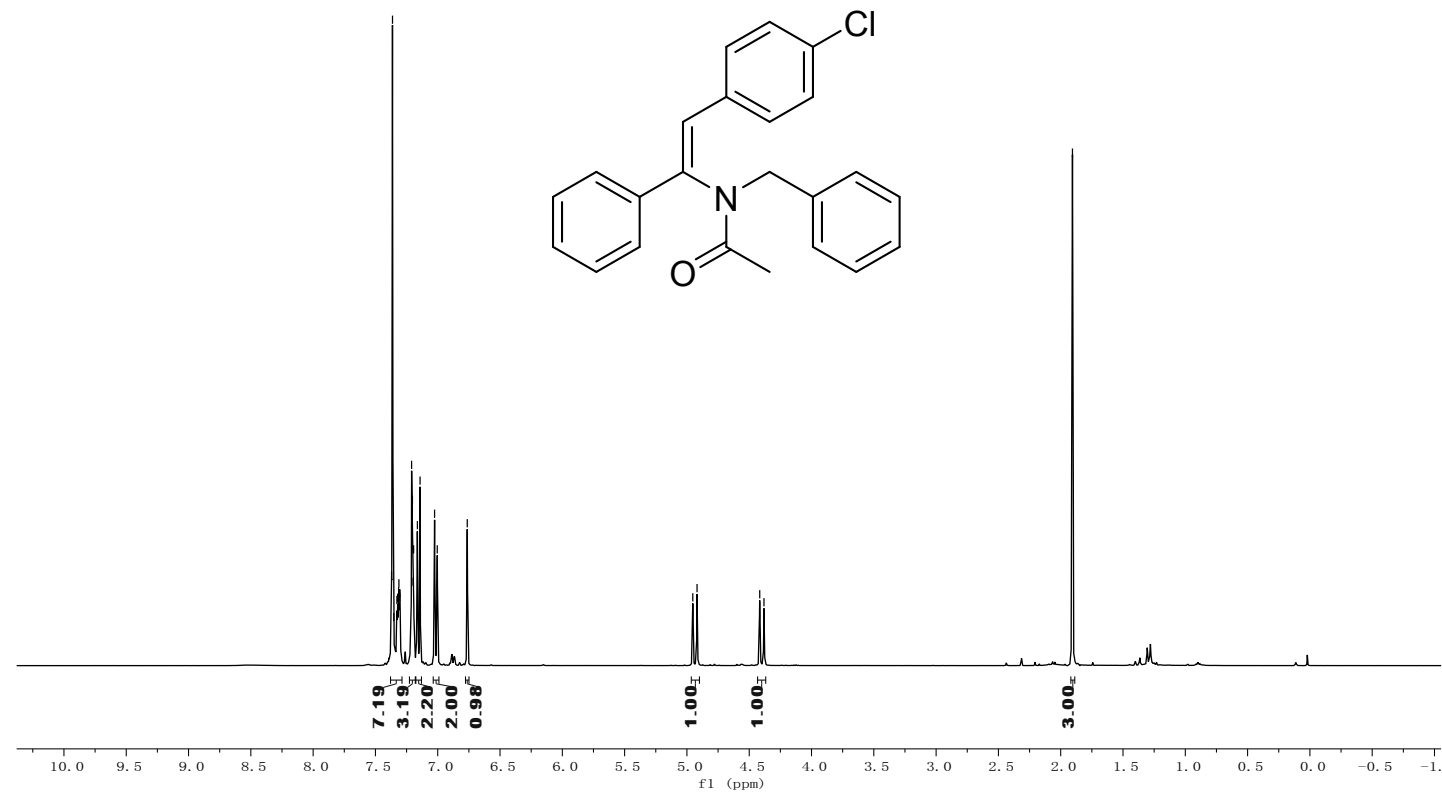

(Z)- $N$-benzyl- $N$-(2-(4-chlorophenyl)-1-phenylvinyl)acetamide (4I)

${ }^{13} \mathrm{C}$ NMR (101 MHz, $\left.\mathrm{CDCl}_{3}\right)$

$\begin{array}{ll}1 & \\ 0 & 0\end{array}$

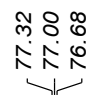

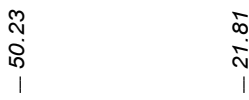

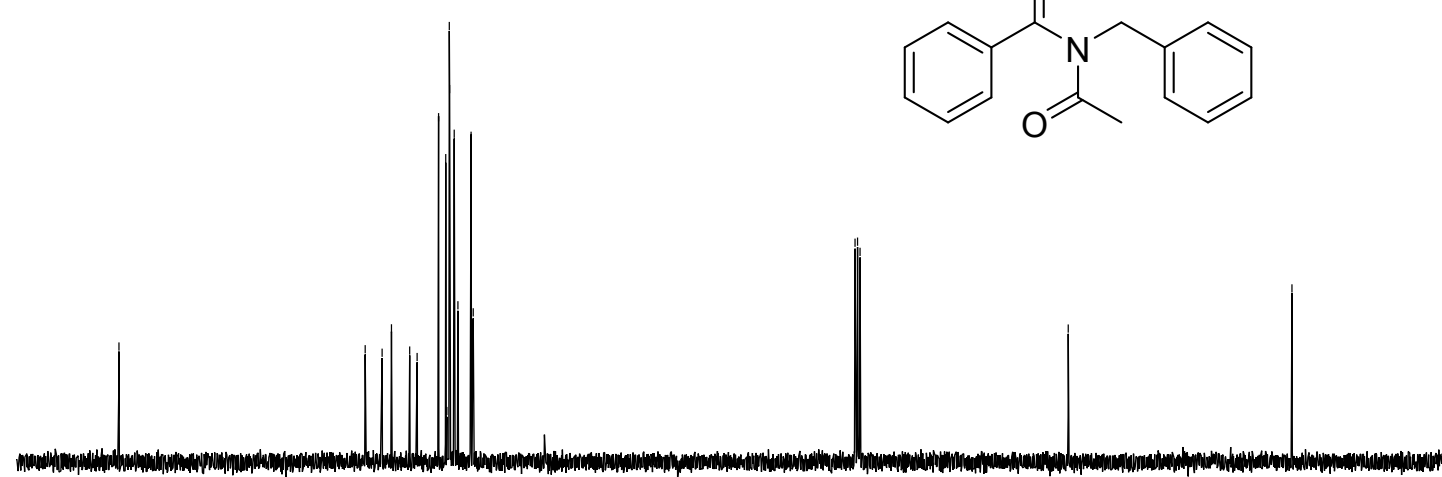<smiles>CC(=O)N(Cc1ccccc1)/C(=C\c1ccc(Cl)cc1)c1ccccc1</smiles>

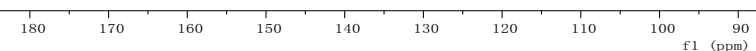

(Z)- $N$-benzyl- $N$-(1-phenyl-2-(4-(trifluoromethyl)phenyl)vinyl)acetamide (4m) ${ }^{1} \mathrm{H} \mathrm{NMR}\left(400 \mathrm{MHz}, \mathrm{CDCl}_{3}\right)$ 


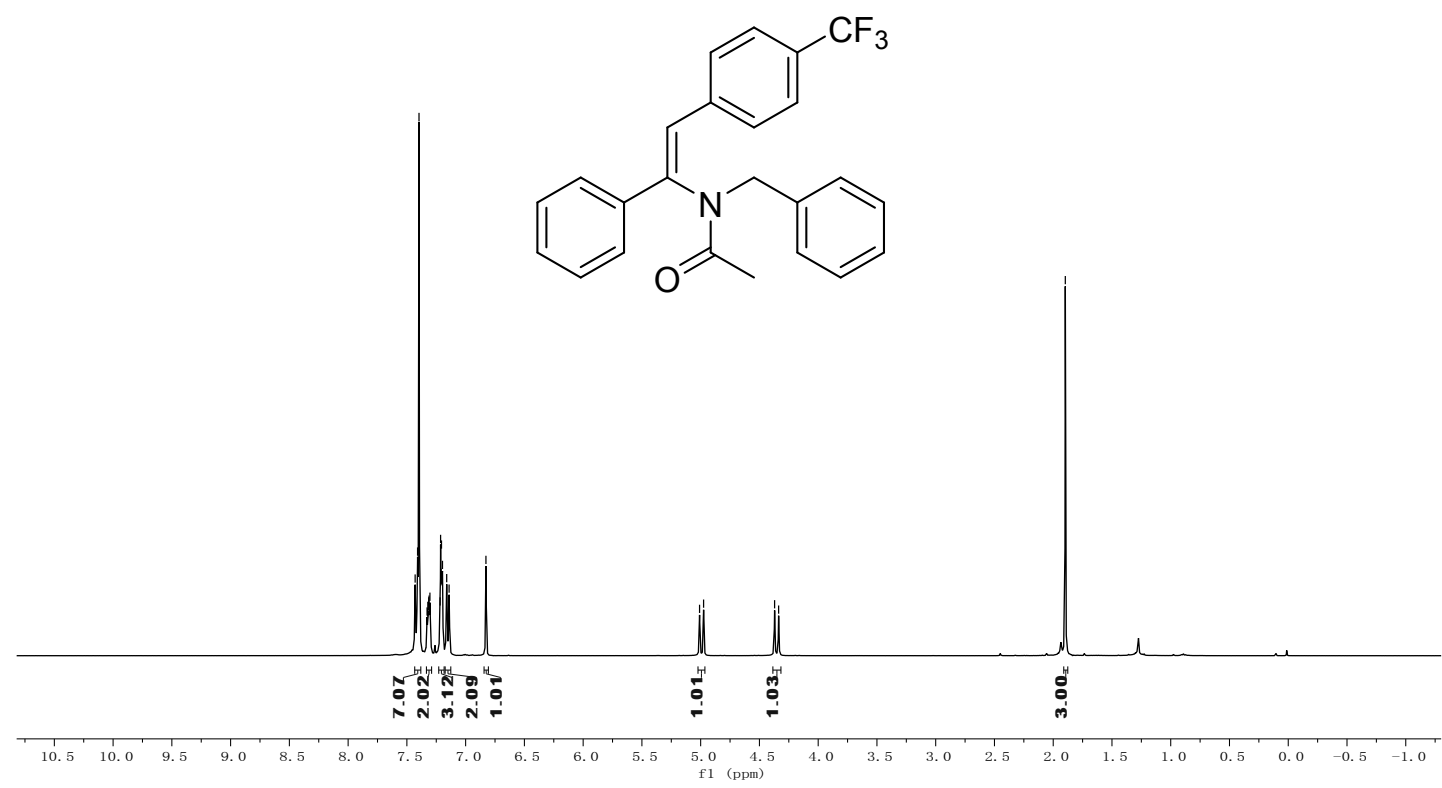

(Z)- $N$-benzyl- $N$-(1-phenyl-2-(4-(trifluoromethyl)phenyl)vinyl)acetamide (4m) ${ }^{13} \mathrm{C}$ NMR (101 MHz, $\left.\mathrm{CDCl}_{3}\right)$

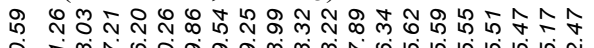

ํ.

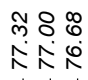

m

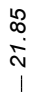<smiles>CC(=O)N(Cc1ccccc1)/C(=C\c1ccc(C(F)(F)F)cc1)c1ccccc1</smiles>
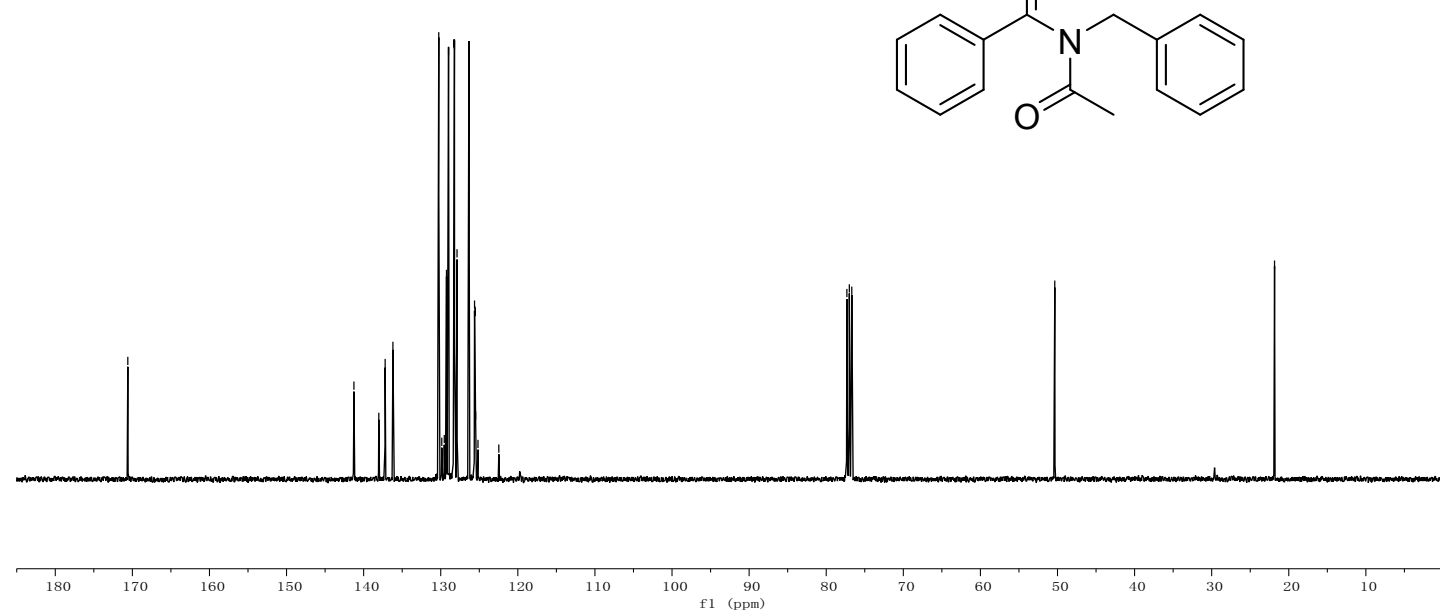

(Z)- $N$-(2-([1,1'-biphenyl]-4-yl)-1-phenylvinyl)- $N$-benzylacetamide (4n)

${ }^{1} \mathrm{H}$ NMR $\left(400 \mathrm{MHz}, \mathrm{CDCl}_{3}\right)$ 


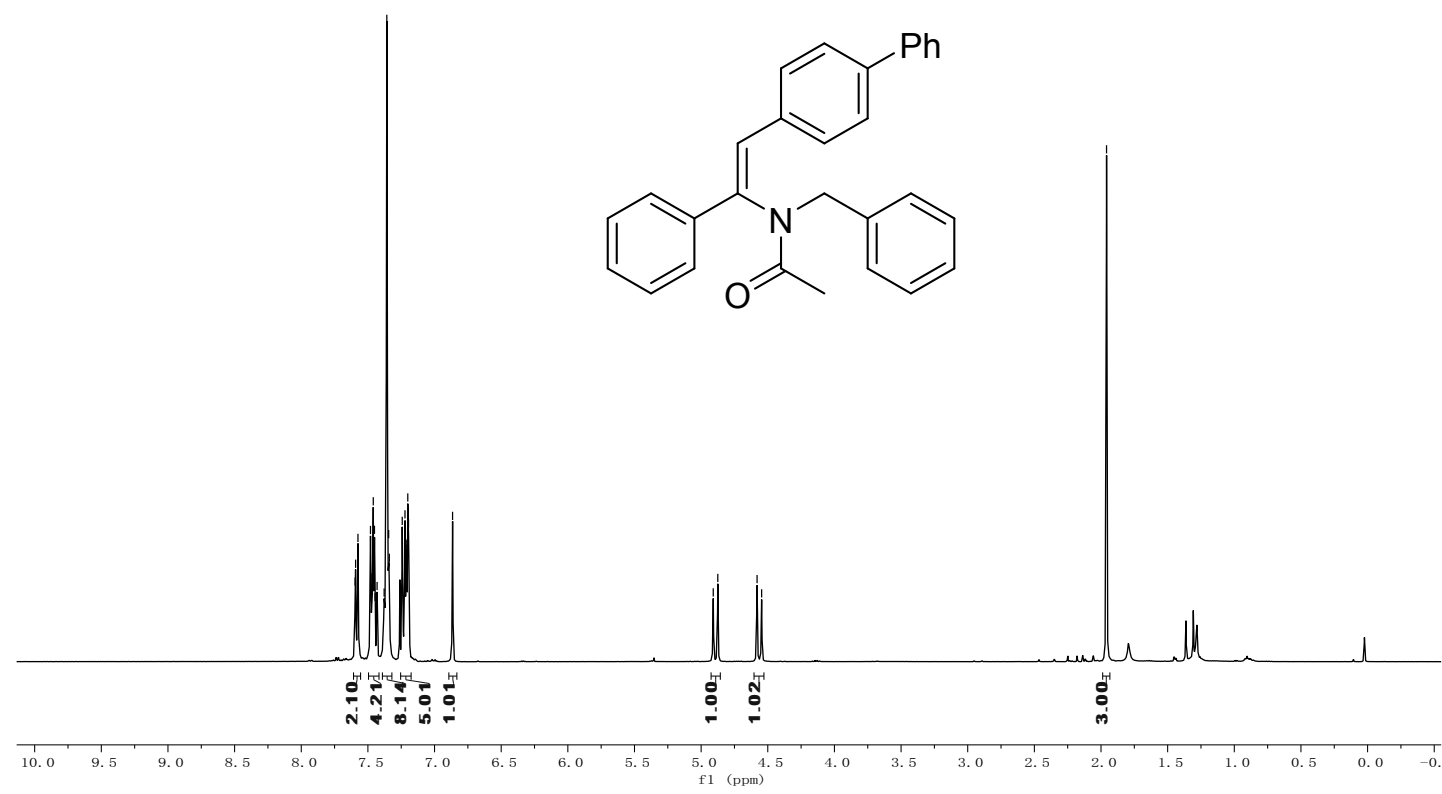

(Z)- $N$-(2-([1,1'-biphenyl]-4-yl)-1-phenylvinyl)- $N$-benzylacetamide (4n) ${ }^{13} \mathrm{C}$ NMR $\left(101 \mathrm{MHz}, \mathrm{CDCl}_{3}\right)$

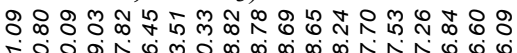

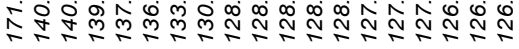
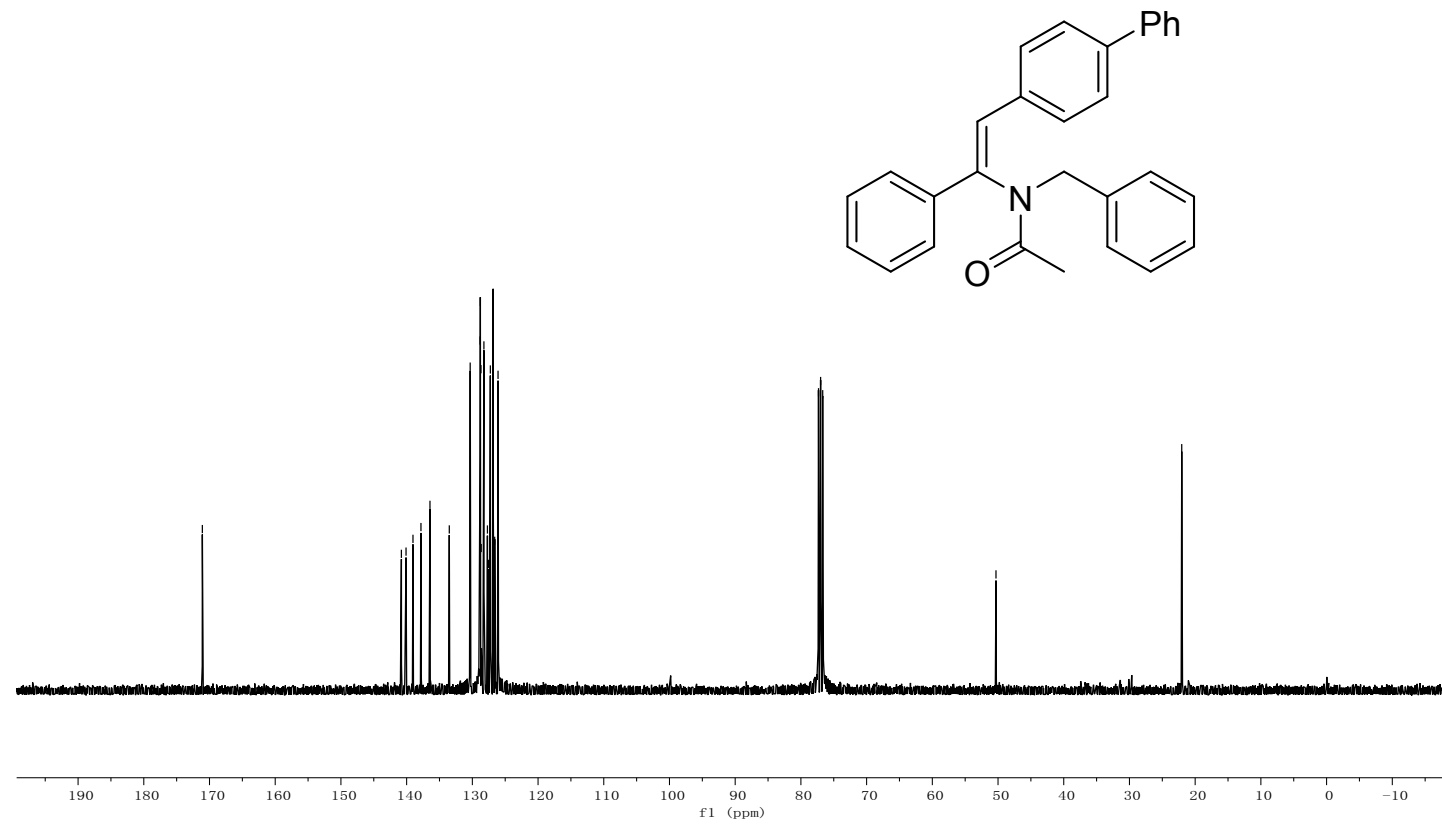

(Z)- $N$-benzyl- $N$-(2-(naphthalen-1-yl)-1-phenylvinyl)acetamide (4o) ${ }^{1} \mathrm{H}$ NMR $\left(400 \mathrm{MHz}, \mathrm{CDCl}_{3}\right)$ 


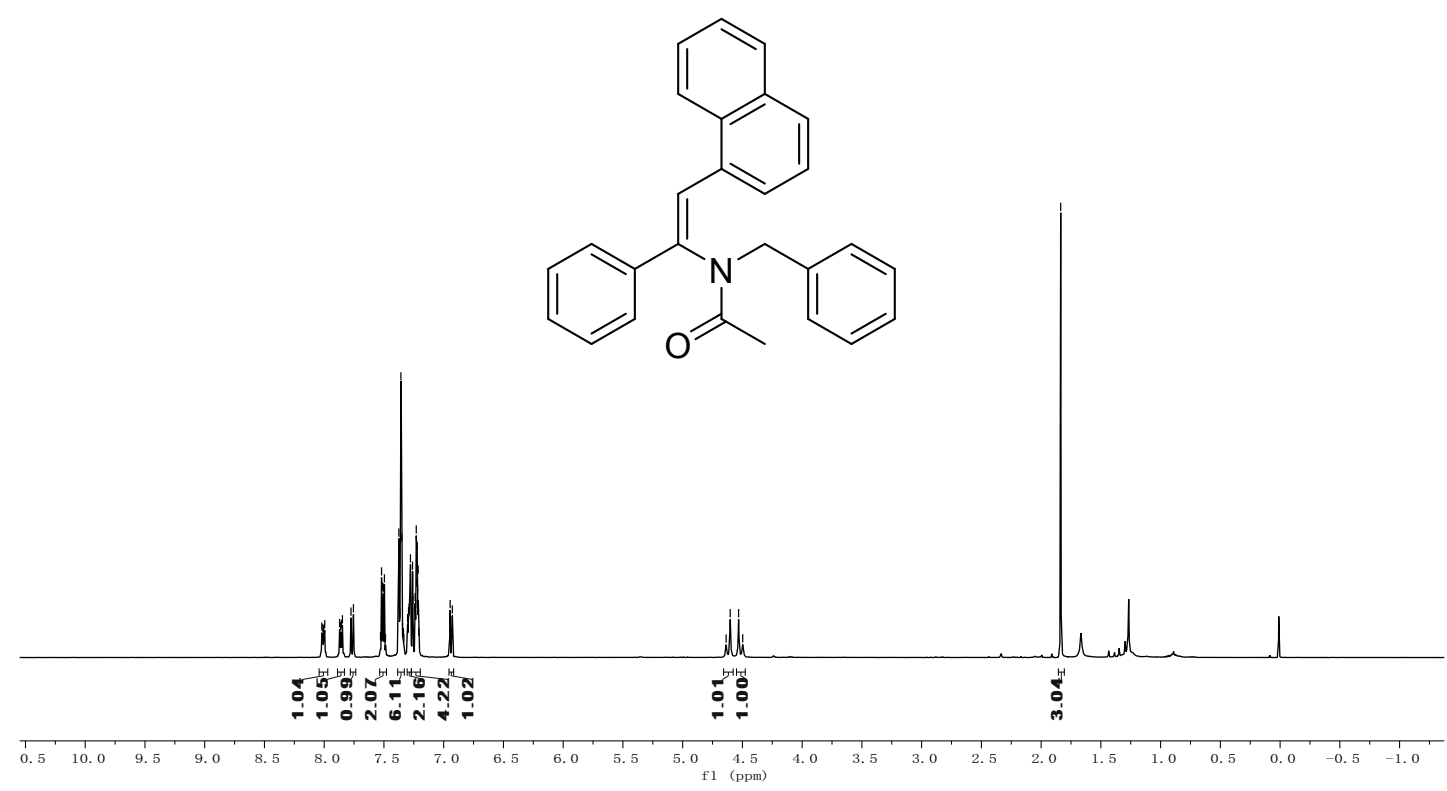

(Z)- $N$-benzyl- $N$-(2-(naphthalen-1-yl)-1-phenylvinyl)acetamide (4o)

${ }^{13} \mathrm{C} \mathrm{NMR}\left(101 \mathrm{MHz}, \mathrm{CDCl}_{3}\right)$

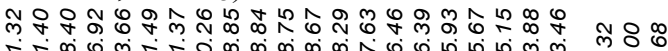

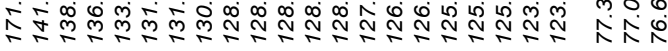

$\begin{array}{ll}4 & \text { ก } \\ 0 & \text { N } \\ 0 & 1\end{array}$

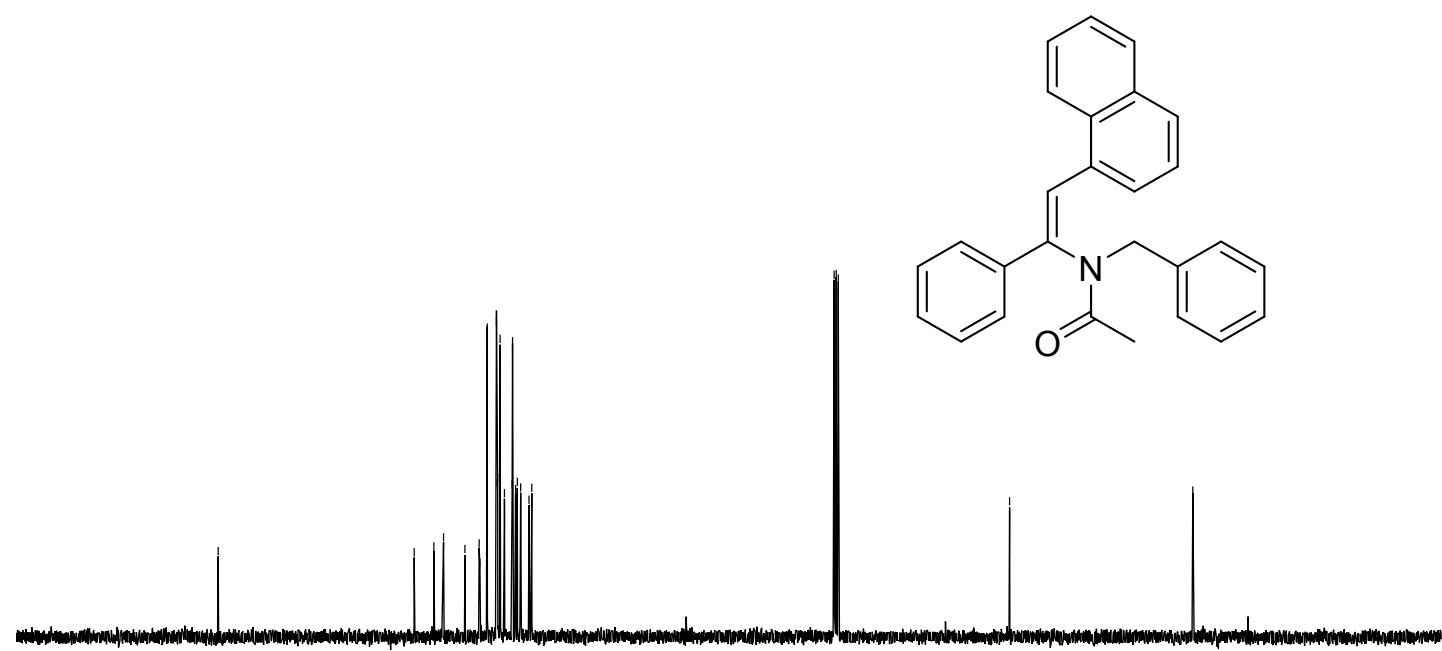

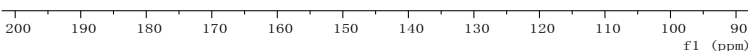

(Z)- $N$-benzyl- $N$-(1-phenyl-2-(thiophen-3-yl)vinyl)acetamide (4p)

${ }^{1} \mathrm{H}$ NMR (400 MHz, $\left.\mathrm{CDCl}_{3}\right)$ 


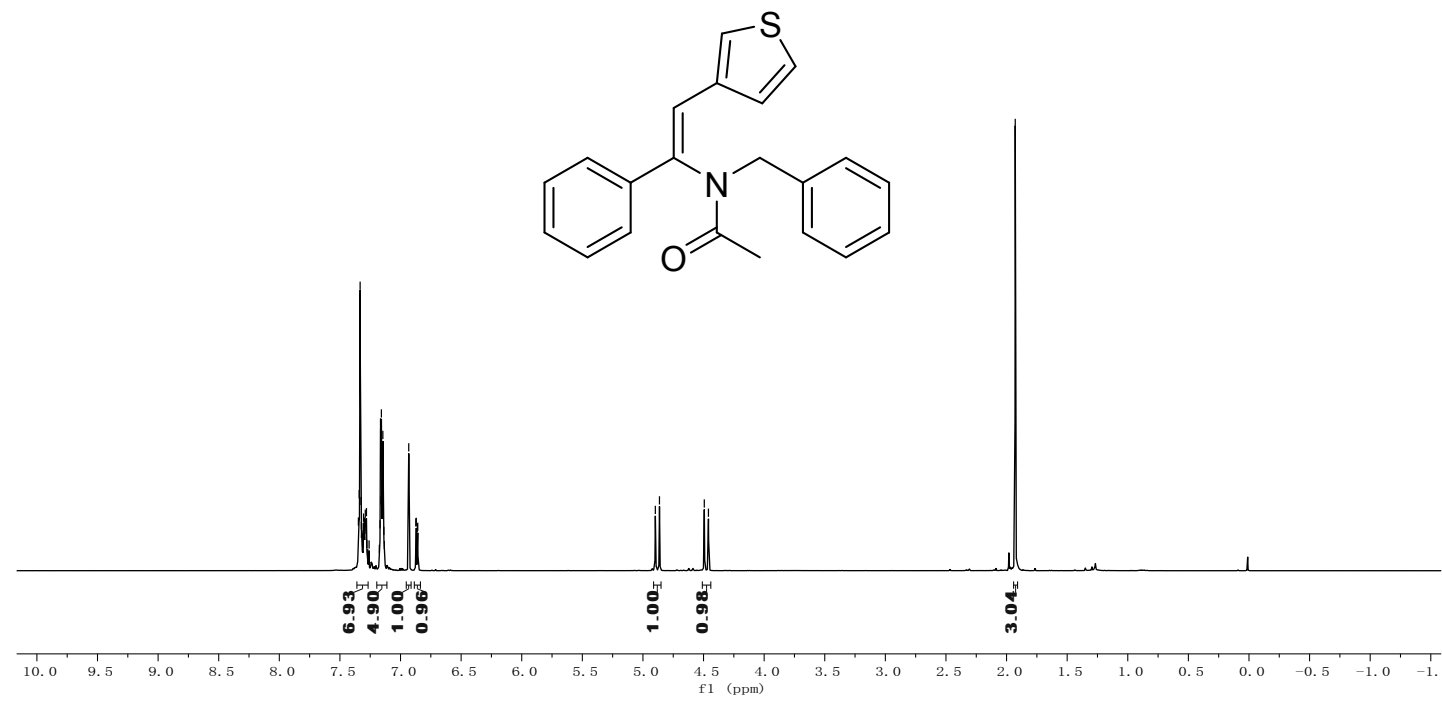

(Z)- $N$-benzyl- $N$-(1-phenyl-2-(thiophen-3-yl)vinyl)acetamide (4p) ${ }^{13} \mathrm{C}$ NMR (101 MHz, $\left.\mathrm{CDCl}_{3}\right)$

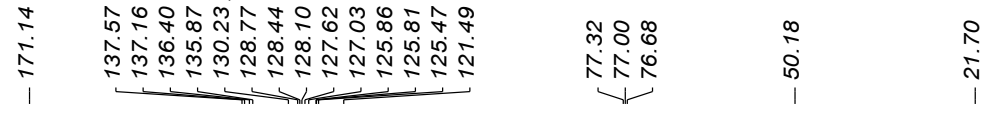

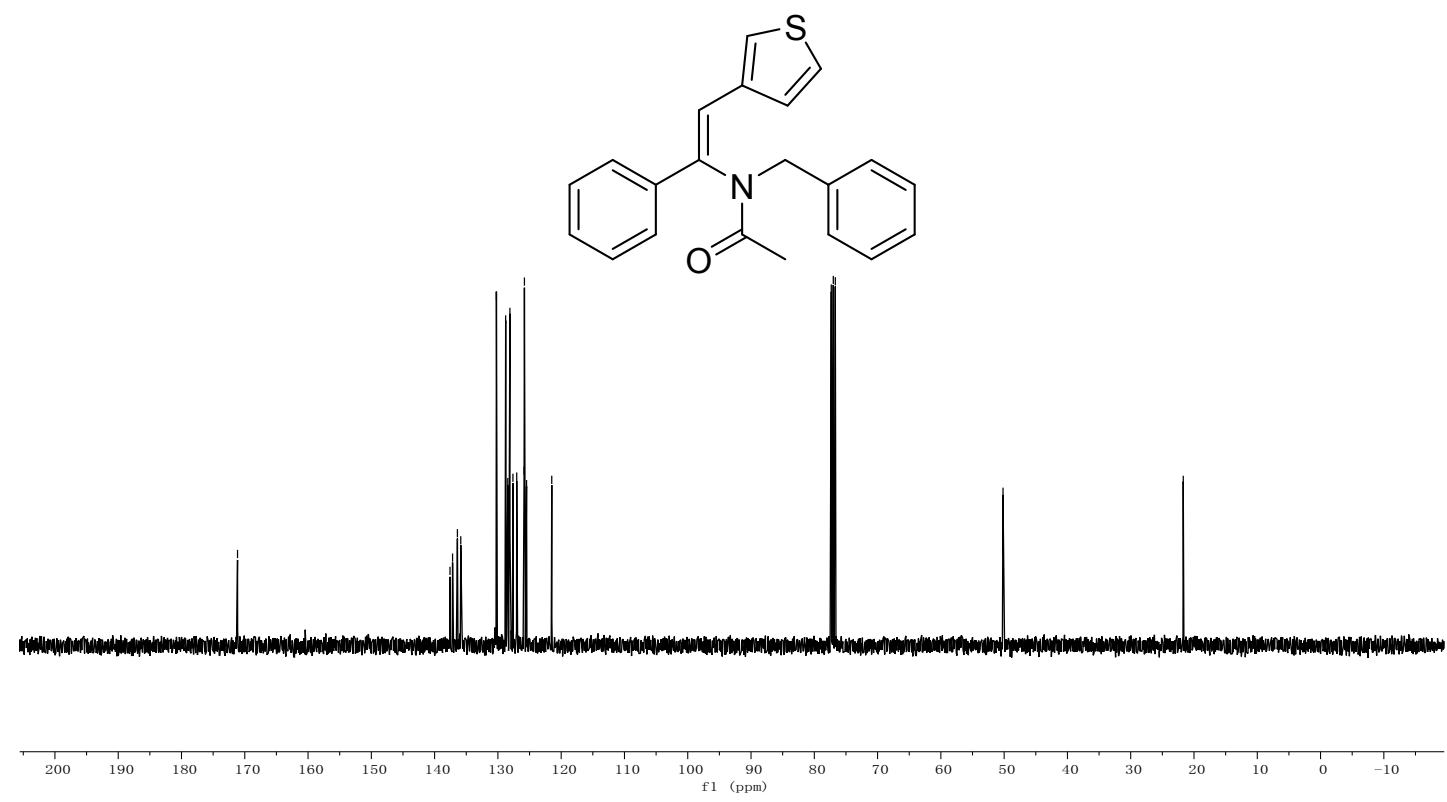

$N$-benzyl- $N$-(1,2-diphenylethyl)acetamide (5)

${ }^{1} \mathrm{H}$ NMR $\left(400 \mathrm{MHz}, \mathrm{CDCl}_{3}\right)$ 


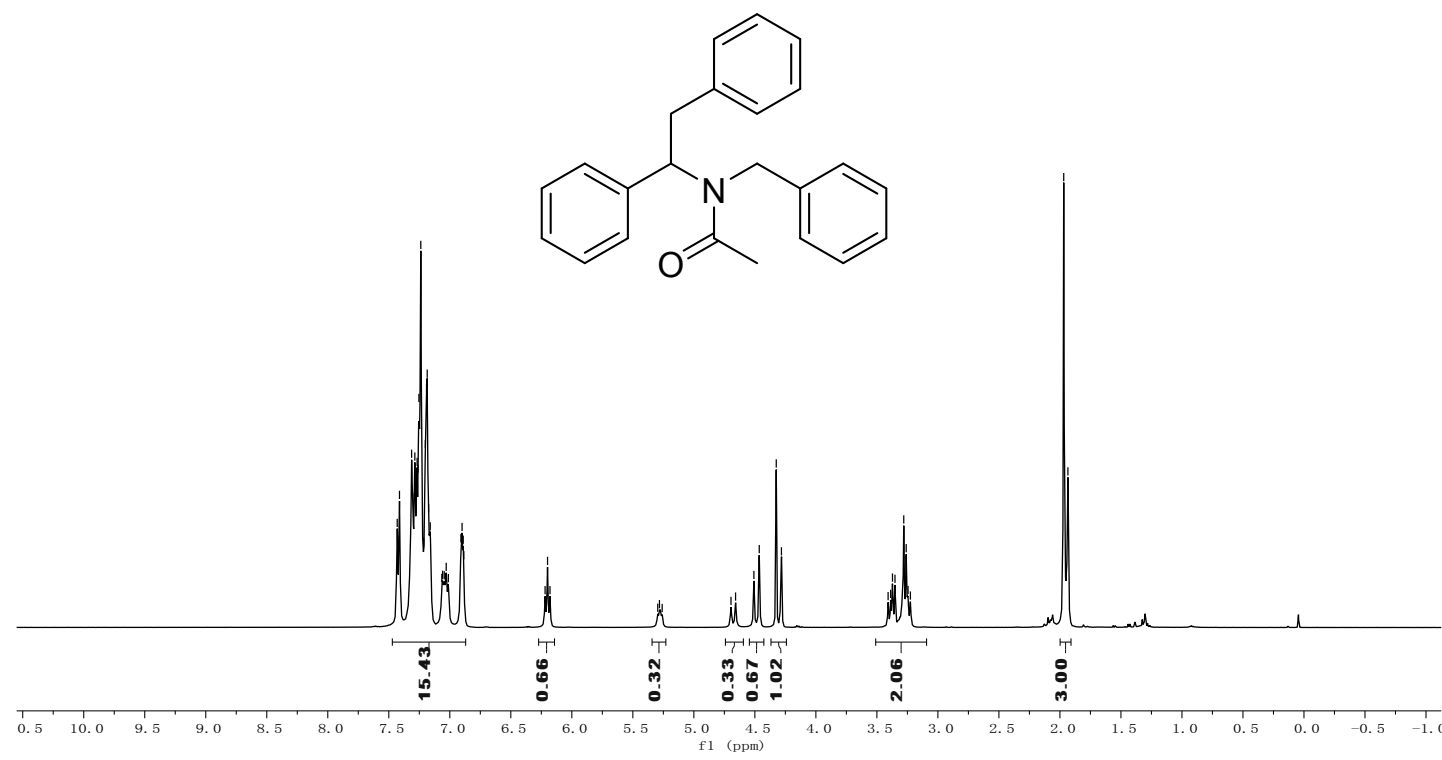

$N$-benzyl- $N$-(1,2-diphenylethyl)acetamide (5)

${ }^{13} \mathrm{C}$ NMR (101 MHz, $\left.\mathrm{CDCl}_{3}\right)$

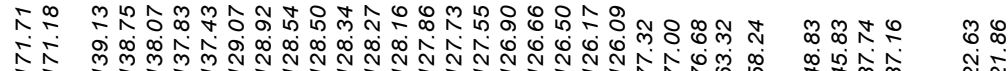

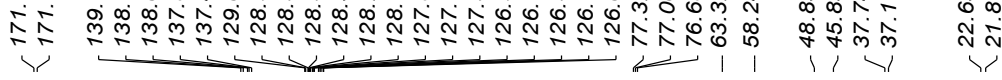
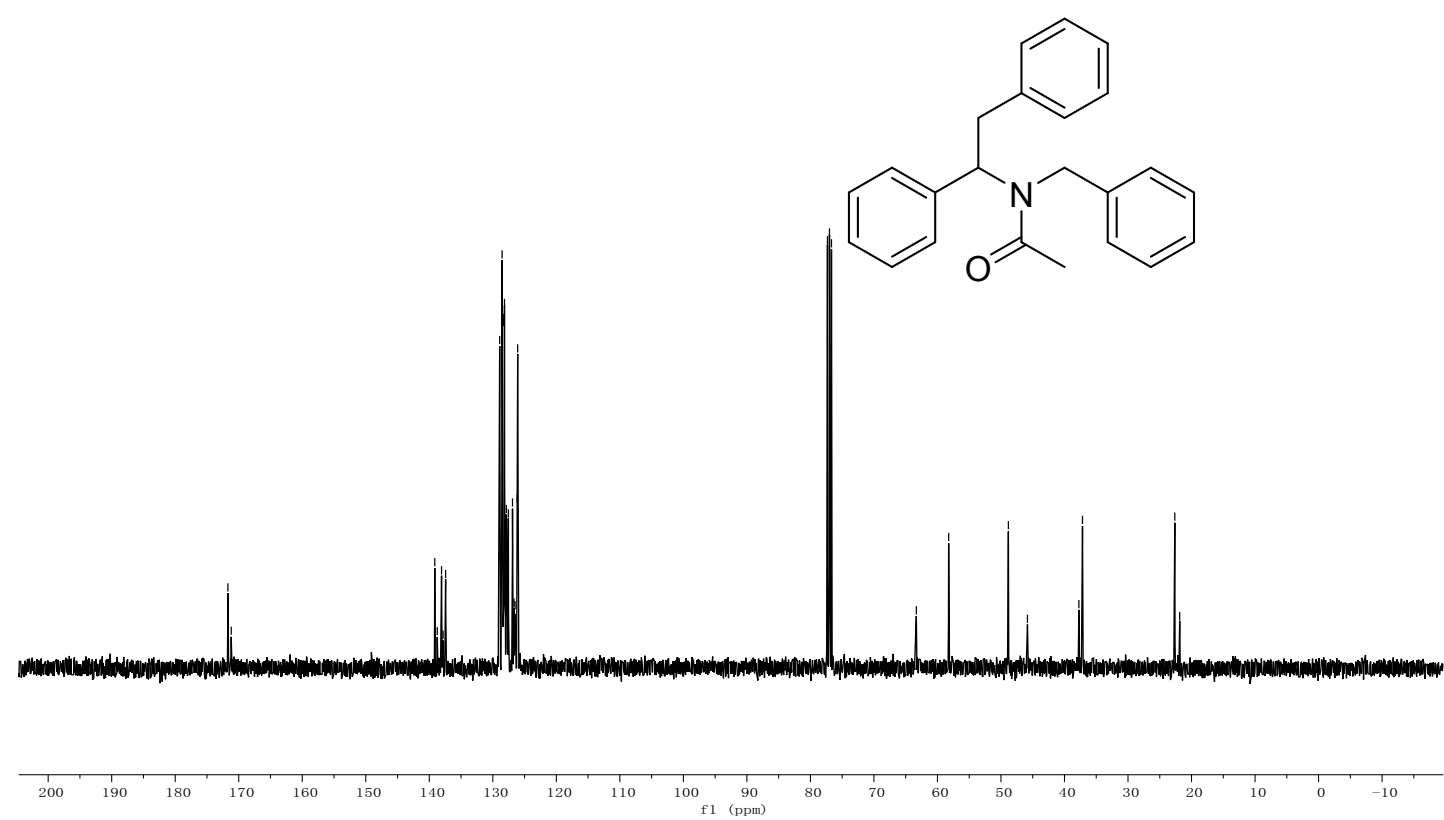

$N$-benzyl- $N$-(1,2,2-triphenylvinyl)acetamide (6)

${ }^{1} \mathrm{H}$ NMR (400 MHz, $\left.\mathrm{CDCl}_{3}\right)$ 


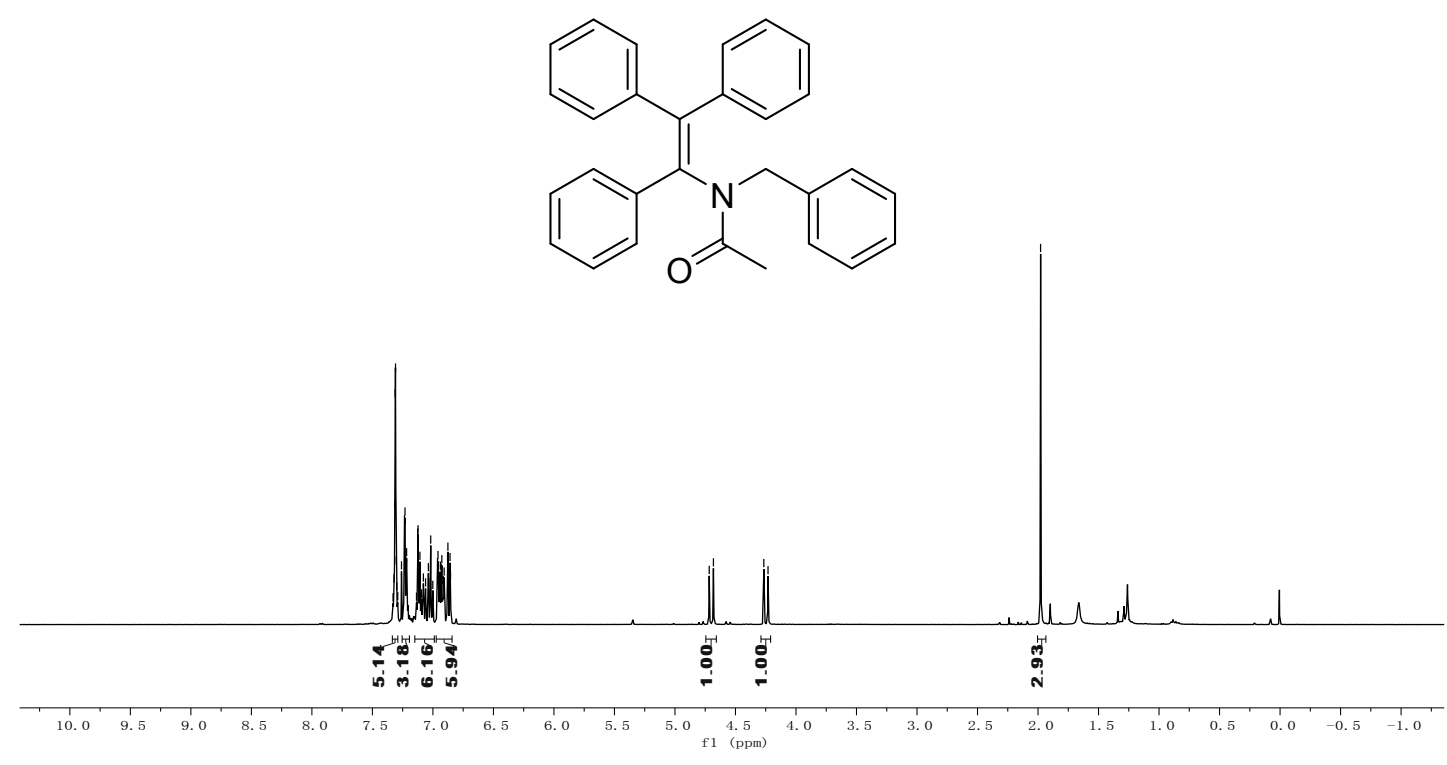

$N$-benzyl- $N$-(1,2,2-triphenylvinyl)acetamide (6) ${ }^{13} \mathrm{C}$ NMR (101 MHz, $\left.\mathrm{CDCl}_{3}\right)$

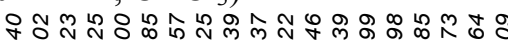

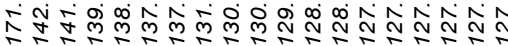

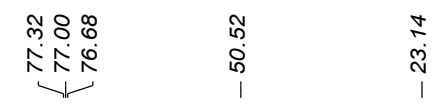<smiles>CC(=O)N(Cc1ccccc1)C(=C(c1ccccc1)c1ccccc1)c1ccccc1</smiles>
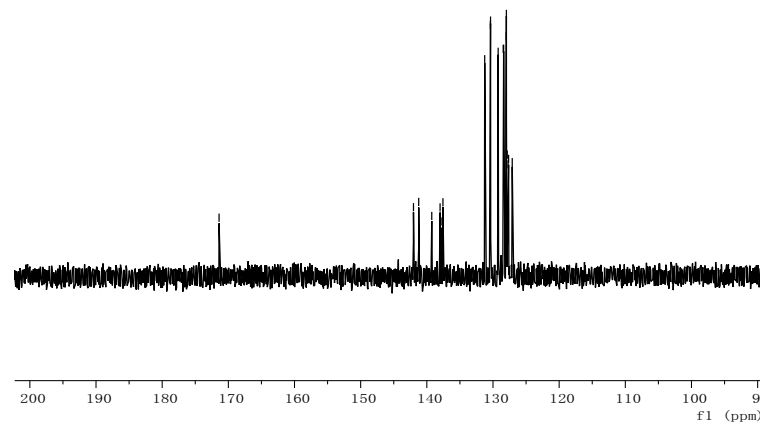